\title{
Parametric Instability Rates in Periodically Driven Band Systems
}

\author{
S. Lellouch, ${ }^{1, *}$ M. Bukov, ${ }^{2}$ E. Demler, ${ }^{3}$ and N. Goldman ${ }^{1, \dagger}$ \\ ${ }^{1}$ Center for Nonlinear Phenomena and Complex Systems, Université Libre de Bruxelles, \\ CP 231, Campus Plaine, B-1050 Brussels, Belgium \\ ${ }^{2}$ Department of Physics, Boston University, \\ 590 Commonwealth Avenue, Boston, Massachusetts 02215, USA \\ ${ }^{3}$ Department of Physics, Harvard University, Cambridge, Massachusetts 02138, USA
}

(Received 20 October 2016; revised manuscript received 18 February 2017; published 5 May 2017)

\begin{abstract}
In this work, we analyze the dynamical properties of periodically driven band models. Focusing on the case of Bose-Einstein condensates, and using a mean-field approach to treat interparticle collisions, we identify the origin of dynamical instabilities arising from the interplay between the external drive and interactions. We present a widely applicable generic numerical method to extract instability rates and link parametric instabilities to uncontrolled energy absorption at short times. Based on the existence of parametric resonances, we then develop an analytical approach within Bogoliubov theory, which quantitatively captures the instability rates of the system and provides an intuitive picture of the relevant physical processes, including an understanding of how transverse modes affect the formation of parametric instabilities. Importantly, our calculations demonstrate an agreement between the instability rates determined from numerical simulations and those predicted by theory. To determine the validity regime of the mean-field analysis, we compare the latter to the weakly coupled conserving approximation. The tools developed and the results obtained in this work are directly relevant to present-day ultracold-atom experiments based on shaken optical lattices and are expected to provide an insightful guidance in the quest for Floquet engineering.
\end{abstract}

DOI: 10.1103/PhysRevX.7.021015

\section{INTRODUCTION}

Periodically driven systems have been widely studied over the past decades, revealing rich and exotic phenomena in a large class of physical systems. Some of the original applications include examples from classical mechanics, e.g., the periodically kicked rotor [1] and the Kapitza pendulum [2], which display fascinating effects, such as dynamical stabilization [3] or integrability to chaos transitions. More recently, studies of their quantum counterparts have led to remarkable applications in a wide range of physical platforms, such as ion traps [4], photonic crystals [5], irradiated graphene [6-8] and ultracold gases in optical lattices $[9,10]$.

A major reason for the renewed interest in periodically driven quantum systems is the fact that they constitute the essential ingredient for Floquet engineering [6,9-12]. To see this, recall that periodically driven quantum systems obey Floquet's theorem [13], which implies that the timeevolution operator $\hat{U}(t, 0)$ of a system, whose dynamics is

\footnotetext{
*samuel.lellouch@ulb.ac.be

†ngoldman@ulb.ac.be
}

Published by the American Physical Society under the terms of the Creative Commons Attribution 4.0 International license. Further distribution of this work must maintain attribution to the author(s) and the published article's title, journal citation, and DOI.
Subject Areas: Atomic and Molecular Physics, Quantum Physics, Superfluidity generated by the Hamiltonian $\hat{H}(t+T)=\hat{H}(t)$, can be written as $[11,14]$

$$
\begin{aligned}
\hat{U}(t, 0) & =\mathcal{T} \exp \left(-i \int_{0}^{t} \mathrm{~d} t^{\prime} \hat{H}\left(t^{\prime}\right)\right) \\
& =\mathrm{e}^{-i \hat{K}_{\text {kick }}(t)} \mathrm{e}^{-i t \hat{H}_{\text {eff }}} \mathrm{e}^{i \hat{K}_{\text {kick }}(0)},
\end{aligned}
$$

where $\mathcal{T}$ denotes time ordering. Here, we introduced the time-independent effective Hamiltonian $\hat{H}_{\text {eff }}$, as well as the time-periodic "kick" operator $\hat{K}_{\text {kick }}(t+T)=\hat{K}_{\text {kick }}(t)$, which has zero average over one driving period [11]. It then follows that the stroboscopic time evolution $(t=N T$, $N \in \mathbb{Z}$ ) of such systems is governed by

$$
\begin{aligned}
\hat{U}(N T, 0) & =\mathrm{e}^{-i \hat{K}_{\text {kick }}(0)} \mathrm{e}^{-i N T \hat{H}_{\text {eff }}} \mathrm{e}^{i \hat{K}_{\text {kick }}(0)}=\mathrm{e}^{-i N T \hat{H}_{\mathrm{F}}}, \\
\hat{H}_{\mathrm{F}} & =\mathrm{e}^{-i \hat{K}_{\text {kick }}(0)} \hat{H}_{\text {eff }} \mathrm{e}^{i \hat{K}_{\text {kick }}(0)} .
\end{aligned}
$$

Hence, up to a change of basis [15], the stroboscopic time evolution is completely described by the effective Hamiltonian $\hat{H}_{\text {eff }}$, which can be designed by suitably tailoring the driving protocol [11,12,16-18]. Moreover, we note that the dynamics taking place within each period of the drive, the so-called micromotion, is entirely captured by the kick operator $\hat{K}_{\text {kick }}(t)$. On the theoretical side, perturbative methods to compute both the effective Hamiltonian and the micromotion operators have been 
developed in the form of an inverse-frequency expansion $[11,14,16,19]$, variants of which include the FloquetMagnus [12,20], the van Vleck [17], and the BrillouinWigner [18] expansions.

On the experimental side, Floquet engineering has been used to explore a wide variety of physical phenomena, ranging from dynamical trapping of ions in Paul traps [4] to one-way propagating states in photonic crystals [5]. In the context of ultracold quantum gases, this concept soon resonated with the idea of quantum simulation [21], as it became clear that Floquet engineering could offer powerful schemes to reach novel models and properties, typically inaccessible in conventional condensed-matter materials $[9,10]$. For instance, shaken optical lattices have been used to explore dynamical localization [22-26], photon-assisted tunneling [27,28], and driven-induced superfluid-to-Mottinsulator transitions [29-32]. In the field of disordered quantum systems, the periodically kicked rotor has been exploited as a flexible and successful simulator for Anderson localization [33-35]. More recently, a series of experiments implemented shaken or time-modulated optical-lattice potentials in view of designing artificial gauge fields for neutral atoms [36-42], some of which led to nontrivial topological band structures [9] or frustrated quantum models $[43,44]$. Moreover, an experiment demonstrated the control over the population of targeted Floquet states [45]. Other applications of Floquet engineering in cold atoms include the experimental realization of state-dependent lattices [46] and dynamical topological phase transitions [47], as well as proposals to dynamically stimulate quantum coherence [48] or to create subwavelength optical lattices [49], synthetic dimensions [50], or pure Dirac-type dispersions [11,51]. In an even broader context, Floquet engineering is at the core of photo-induced superconductivity [52-54] and topological insulators [55], and time crystals [56-58].

Today, the theory of periodically driven quantum systems (including the resulting artificial gauge fields and topological band structures $[9,10]$ ) is well established for single particles, and there is a strong need for incorporating interparticle interactions in the description of such systems. Indeed, interparticle interactions constitute an essential ingredient in simulating condensed-matter systems [21], and the relevance of many theoretical proposals strongly relies on the extent to which the systems of interest remain stable away from the noninteracting limit. Moreover, in their quest for realizing new topological states of matter, experiments based on driven quantum systems are more than ever eager to consider interacting systems [59]; indeed, building on the successful optical-lattice implementation of flat energy bands with nontrivial Chern numbers [39], mastering interactions in this context would allow for the engineering of intriguing strongly correlated states, such as fractional Chern insulators [60]. In this framework, we point out that a useful tool to derive the effective low-energy physics of strongly correlated systems, namely, the Schrieffer-Wolff transformation, was generalized to periodically driven systems [61].

However, until now, the presence of interparticle interactions has led to complications. Recent experiments on time-modulated ("shaken") optical lattices [36-39,62] have reported severe heating, particle loss, and dissipative processes, whose origin presumably stems from a rich interplay between the external drive and interparticle collisions. In this sense, understanding the role of interactions in driven systems, and their relation to heating processes and instabilities, is both of fundamental and practical importance. On the theory side, several complementary approaches have been considered to tackle the problem. On the one hand, a perturbative scattering theory, leading to a so-called "Floquet Fermi golden rule" [63,64], has been developed to estimate heating and loss rates, and it showed good agreement with the band-population dynamics reported in Ref. [39]. Extensions to Bose-Einstein condensates (BEC) have been proposed [65,66]; however, to the best of our knowledge, the resulting loss rates have not yet been confirmed by experiments or by numerical simulations. On the other hand, various studies analyzed the dynamical instabilities that occur in BEC trapped in moving, shaken, or time-modulated optical lattices [67-73]. For instance, Ref. [73] analyzed such dynamical instabilities through a numerical analysis of the Bogoliubov-de Gennes (BdG) equations; however, no link was made with heating processes, and the approach lacked a conclusive comparison with analytics. Importantly, several studies pointed out the parametric nature of those instabilities $[70,71,74,75]$. Interestingly, we note that parametric instabilities, and their impact on energy absorption and thermalization processes, have also been studied theoretically in a wider context, ranging from Luttinger liquids [76] to cosmology [77-79]. In the context of driven optical lattices, the parametric amplification of scattered atom pairs, through phase-matching conditions involving the band structure and the drive, has also been identified [80-83]. Altogether, there is a strong need for a combined analytical and numerical study of driven optical-lattice models, which would provide analytical estimates of instability and heating rates supported by numerical simulations.

\section{A. Scope of the paper}

The object of the present paper is to explore the physics of periodically driven bosonic band models, where interparticle interactions are treated within mean-field (Bogoliubov) theory. Indeed, as in Refs. [65,66,73,75], we assume that the driven atomic gas forms a BEC, and we analyze the properties of the corresponding Bogoliubov excitations in response to the external drive. To do so, we develop a generic and widely applicable method to investigate the short-time dynamics of periodically driven 
bosonic systems and to extract the corresponding instability rates, both numerically and analytically.

We first focus on a shaken one-dimensional (1D) lattice model and extensively explore the occurrence of instabilities and heating in this system, before considering more elaborate models. In a previous study on a similar model [73], a dynamical instability of the condensate was found to occur above a critical interaction strength, which was numerically calculated as a function of the drive amplitude $K$ and frequency $\omega$. This allowed the author to numerically map out the stability boundaries between the stable and unstable phases. More recently, Refs. [74,75] suggested that such boundaries could be understood in terms of a simple energy-conservation criterion, which could be formulated within a parametric-instability analysis. In this work, we offer a significant advance in the description and understanding of the instabilities that affect bosonic periodically driven systems, both qualitatively and quantitatively, as we summarize below:

(1) We show how to obtain the instability rates as a function of the model parameters, both numerically and analytically, and we establish their relation to the growth of physical quantities in the system, such as the energy, thus providing a link between dynamical instability and heating. Moreover, we identify different instability regimes in the system, which are associated with different time scales and characterized by a different behavior in the growth of physical quantities. We introduce several observables that reveal clear signatures of these instabilities, including the noncondensed (depleted) fraction and the momentum distribution of quasiparticle modes (see Fig. 1); we discuss how they could be observed in current ultracold-atom experiments.

(2) We present a full numerical solution of the meanfield problem, determining the stability diagram of the model from first principles (see Fig. 2). We stress that the quantitative character and versatility of our procedure make it general enough to be applicable to a wide class of periodically driven systems, including those involving resonant modulations, higherdimensional lattices, various geometries (e.g., square or honeycomb lattices, continuous space), and spindependent lattices. Moreover, it can also be enriched to incorporate a full experimental sequence, e.g., including adiabatic state preparation [62]. Our results are expected to determine experimentally favorable regimes by providing $a b$ initio numerical (and analytical) estimates for the instability rates in a variety of experimental configurations.

(3) We perform an analytical treatment of the problem based on the existence of parametric resonances in the Bogoliubov-de Gennes equations. In Ref. [74], following a weak-coupling conserving approximation, it was argued that a driven-lattice model was

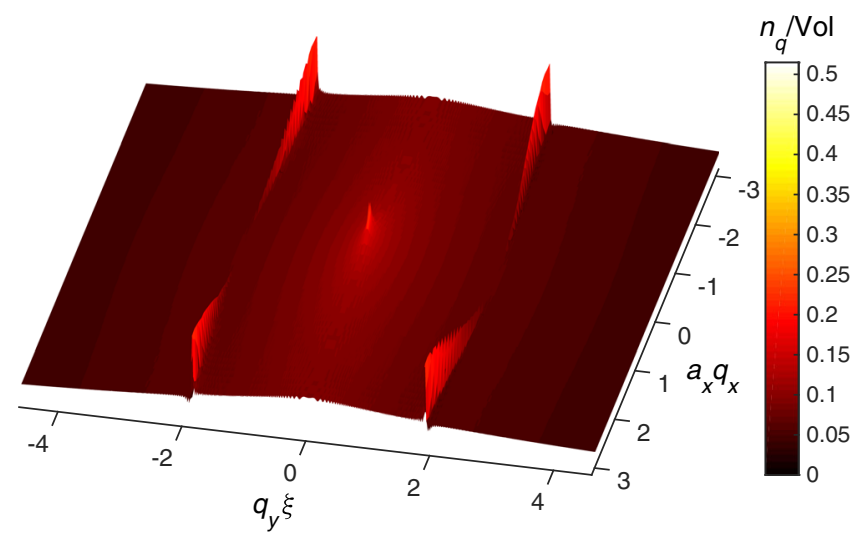

FIG. 1. The quasiparticle momentum distribution $n_{q}$ of a $2 \mathrm{D}$ shaken BEC trapped in a one-dimensional lattice (along $x$ ), and allowed to move along a continuous (tube) transverse direction $(y)$, develops unique resonance structures in the presence of a periodic drive. Dominating the dynamics in the early stage of evolution, they signal the onset of instabilities and provide a clear experimental signature of the parametric resonance phenomenon explained in this work. The momentum distribution is shown after 60 driving periods $(t=60 T)$. Here, the distribution $n_{q}$ is divided by the system's volume Vol, $\xi$ denotes the BEC healing length, and $a_{x}$ is the lattice spacing constant. The precise model and the corresponding parameters are the same as in Fig. 17.

stable against parametric resonance provided that the drive frequency satisfies $\omega>2 W_{\text {eff }}$, with $W_{\text {eff }}$ the bandwidth associated with $\hat{H}_{\text {eff }}$ in the Bogoliubov approximation. Intuitively, this can be understood by recalling that the elementary excitations of the system (i.e., the Bogoliubov phonons) are always created in pairs, and thus, whenever the drive frequency exceeds twice the maximum possible excitation energy, stability is ensured by energy conservation. However, our rigorous analytical derivation reveals that this simple criterion needs to be revised: As we demonstrate, an accurate qualitative explanation requires taking into account both higher-order photon-absorption processes and the detuning from resonance within the parametricresonance treatment [84]. This analysis allows us, for the first time, to derive the functional dependence of the instability growth rate on the model parameters and, ultimately, to understand all the features of the stability diagram (see Fig. 2).

(4) We extend the stability analysis to two-dimensional (2D) models and study the effects due to transverse directions, considering both the case of a transverse lattice (i.e., a discrete transverse degree of freedom) and that of transverse tubes (i.e., a continuous transverse degree of freedom). In the case of continuous degrees of freedom, a theoretical simplification in the equations allows one to obtain 

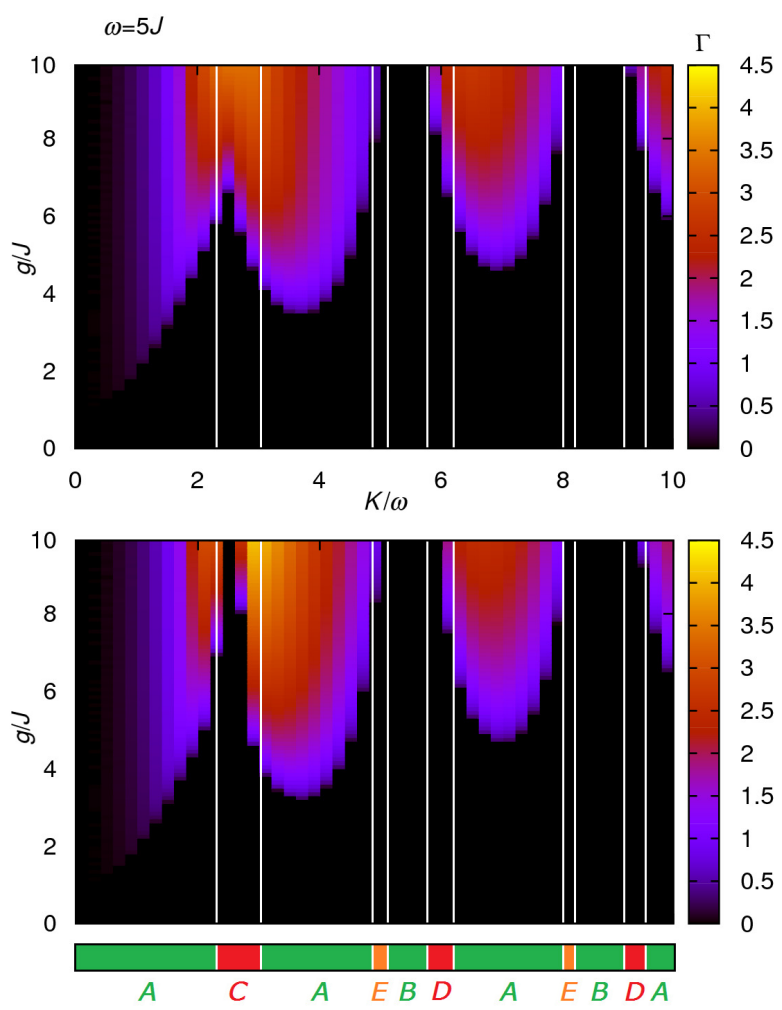

FIG. 2. Numerical (top panel) and analytical (bottom panel) stability diagram for the model described by Eq. (2). The instability rate $\Gamma$ (expressed in units of the hopping amplitude $J$, see text) is plotted as a function of the modulation amplitude $K / \omega$ [defined in Eq. (2)] and interaction strength $g$, for a driving frequency $\omega=5 J$. The vertical lines and letters summarize the agreement and disagreement regions, as discussed in Sec. IV C. A: Away from the zeros of $\mathcal{J}_{0}(K / \omega)$ and $\mathcal{J}_{2}(K / \omega)$, the analytics successfully capture the instability rates extracted from numerics. B: Agreement zone near the (close) zeros of $\mathcal{J}_{0}(K / \omega)$ and $\mathcal{J}_{2}(K / \omega)$, where both analytics and numerics predict a stable behavior. C/D: Close to a zero of $\mathcal{J}_{0}(K / \omega)$, the analytical perturbative approach breaks down. E: Potential quantitative disagreement when the contribution of the second harmonic has a weak amplitude; the instability is then partly due to higher harmonics (see Sec. IV B).

very simple analytical formulas for instability rates, which are in perfect agreement with our full numerical simulations. Moreover, this study provides a clear physical picture of how instabilities are enhanced by the presence of transverse modes in the system, as already anticipated in Refs. [64,66]. More generally, we argue that our theory should provide guidance for experiments; It illustrates, for instance, the advantage of working at high frequency and the necessity of using a strong transverse confinement to reduce parametric instabilities. We also include a discussion of finite-size effects, making a link between the physics of double wells and that of optical lattices.

\section{B. Outline}

This paper is organized as follows. In Sec. II, we derive the general mean-field equations that will constitute the basis of our analysis. Section III is devoted to the numerical solution of those equations, including details on the general procedure used and a presentation of the results, such as the stability diagram of the model under consideration, the identification of several time scales and instability regimes in the problem, and the dynamics of various physical observables. In Sec. IV, we perform an analytical treatment of the problem: Mapping the Bogoliubov equations on a parametric oscillator (Sec. IVA), we build an effective model from which analytical instability rates can be inferred (Sec. IV B). The analytical results are presented in Sec. IV C, including a discussion of the validity regimes of the approach. The case of finite-size systems is presented in Sec. V. In Sec. VI, we discuss the case where a transverse direction is present (a lattice or a continuous one); this includes simple analytical formulas for instability rates as well as a physical understanding of the enhancement of instabilities by transverse modes. Finally, in Sec. VII, we discuss the application of our results to the weakly interacting Bose-Hubbard model in the mean-field regime; in order to understand the role of nonlinear processes and study the regime of validity of our linearized analysis, we employ a weak-coupling conserving approximation to study the leading-order (in the interaction strength) features of particle-conserving dynamics; we identify clear signatures of the instabilities (including the noncondensed fraction and the momentum distribution of quasiparticles) that could be directly probed by current ultracold-atom experiments in modulated optical lattices.

\section{MEAN-FIELD EQUATIONS AND STABILITY ANALYSIS}

Consider a Bose gas in a shaken 1D lattice, with meanfield interactions, governed by the time-dependent GrossPitaevskii equation (GPE) [10,73]

$$
i \partial_{t} a_{n}=-J\left(a_{n+1}+a_{n-1}\right)+K \cos (\omega t) n a_{n}+U\left|a_{n}\right|^{2} a_{n},
$$

where $n$ labels the lattice sites, $J>0$ denotes the tunneling amplitude for nearest-neighbor processes, $U>0$ is the onsite interaction strength, and the time-periodic modulation has an amplitude $K$ and a frequency $\omega=2 \pi / T$. Note that we set $\hbar=1$ throughout this work.

Equation (2) describes a wide variety of physical systems. On the one hand, it is expected to provide a good description for the shaken 1D Bose-Hubbard model [10], when treated in the weakly interacting regime: In this case, the time-modulated system in Eq. (2) can be realized by mechanically modulating an optical lattice filled with weakly interacting bosonic atoms [10]; see also Sec. VII for 
further discussion. On the other hand, some physical systems are exactly described by Eq. (2): for instance, nonlinear optical systems [85], including helical photonic crystal [5]. In all cases, Eq. (2) defines a close self-consistent problem, which constitutes the core of the present study.

Similar to the analysis of Ref. [73], we study the dynamical instabilities of a BEC described by Eq. (2). To do so, we proceed along the following steps:

(i) First, we determine the time evolution of the condensate wave function $a_{n}^{(0)}(t)$ by solving the full time-dependent GPE and assuming that the initial state $a_{n}^{(0)}(t=0)$ forms a BEC. More precisely, our choice for the initial state corresponds to the solution of the static (effective) GPE:

$$
\begin{aligned}
-J_{\text {eff }}\left(a_{n+1}^{(0)}+a_{n-1}^{(0)}\right)+U\left|a_{n}^{(0)}\right|^{2} a_{n}^{(0)} & =\mu a_{n}^{(0)}, \\
J_{\text {eff }} & =J \mathcal{J}_{0}(K / \omega),
\end{aligned}
$$

where we introduced the effective tunneling amplitude $J_{\text {eff }}$ renormalized by the drive [10] and where $\mathcal{J}_{0}$ denotes the zeroth-order Bessel function. For the model under consideration, we find that $a_{n}^{(0)}(t=0)$ is the Bloch state $e^{i p_{0} n}$ of momentum $p_{0}=0$ if $\mathcal{J}_{0}(K / \omega)>0$ (homogeneous condensate) and $p_{0}=$ $\pi$ for $\mathcal{J}_{0}(K / \omega)<0$. Note that such a choice takes into account the initial kick due to the launching of the modulation [11] [86]. Altogether, this choice for the initial state features both the effects of tunneling renormalization and initial launching of the drive, which are both present in our model (2). We emphasize that this prescription for the initial state is the only step of our calculations that relies on the existence of a well-defined high-frequency limit, as provided by the inverse-frequency expansion $[11,14,16]$; indeed, all subsequent results are based on the full time-dependent equations. Note that it is not the purpose of this paper to discuss how to prepare the system in this initial state; one possibility is to use Floquet adiabatic perturbation theory (see Refs. [62,87]), which is also the experimentally preferred strategy.

(ii) Given the time-dependent solution for the condensate wave function $a_{n}^{(0)}(t)$, we analyze its stability by considering a small perturbation

$$
a_{n}(t)=a_{n}^{(0)}(t)\left[1+\delta a_{n}(t)\right]
$$

and linearizing the Gross-Pitaevskii equation (2) in $\delta a_{n}$; we refer to Appendix A for a discussion on the specific parametrization chosen in Eq. (4). This yields the time-dependent Bogoliubov-de Gennes equations, which take the general form

$$
i\left(\begin{array}{c}
\dot{\delta} \dot{a}_{n} \\
\dot{\delta} a_{n}^{*}
\end{array}\right)=\left(\begin{array}{cc}
\mathcal{F}_{n}(t) & U\left|a_{n}^{(0)}\right|^{2} \\
-U\left|a_{n}^{(0)}\right|^{2} & -\mathcal{F}_{n}(t)^{*}
\end{array}\right)\left(\begin{array}{c}
\delta a_{n} \\
\delta a_{n}^{*}
\end{array}\right),
$$

where we introduced the operator $\mathcal{F}_{n}(t)$, whose action on $\delta a_{n}$ is defined by

$$
\begin{aligned}
\mathcal{F}_{n}(t) \delta a_{n} \equiv & -J \frac{\hat{L}\left(a^{(0)} \delta a_{n}\right)_{n}}{a_{n}^{(0)}}+2 U\left|a_{n}^{(0)}\right|^{2} \delta a_{n} \\
& +K \cos (\omega t) n \delta a_{n}-i \frac{\dot{a}_{n}^{(0)}}{a_{n}^{(0)}} \delta a_{n}
\end{aligned}
$$

and where the discrete operator $\hat{L}$ is defined by $\hat{L}(\cdot)_{n} \equiv(\cdot)_{n+1}+(\cdot)_{n-1}$.

At this stage, let us emphasize that the Bogoliubov equations (5) contain the complete time dependence of the problem, which includes the effects related to the micromotion [note that the BEC wave function $a_{n}^{(0)}(t)$ is computed exactly, not stroboscopically]. Importantly, and as will become apparent below in Sec. IV, the full-time dependence of the system plays a crucial role in its stability.

The Bogoliubov equations of motion (5) can be time evolved over one driving period $T$, which allows one to determine the associated "time-evolution" (propagator) matrix $\Phi(T)$. From this, we extract the "Lyapunov" exponents $\epsilon_{q}$, which are related to the eigenvalues $\lambda_{q}$ of $\Phi(T)$ by $\lambda_{q}=\mathrm{e}^{-i \epsilon_{q} T}$. The appearance of Lyapunov exponents with positive imaginary parts thus indicates a dynamical instability [71,73], i.e., an exponential growth of the associated modes at the rate $s_{q}=\operatorname{Im} \epsilon_{q}$, where $s_{q}$ denotes the growth rate of the momentum mode $q$. As we shall see later, a quantitative indicator of the instability is the maximum growth rate of the spectrum,

$$
\Gamma(J, U, K, \omega) \equiv \max _{q} s_{q},
$$

which, in the following, will be referred to as the instability rate $\Gamma$. This choice will be justified in Sec. III B. We point out that the so-defined instability rate is independent of the reference frame (or gauge) and that it is associated with the stroboscopic dynamics ( $t=T \times$ integer), by construction.

For the model under investigation, one can considerably simplify the problem by working in a rotating frame, in which translational invariance is manifest. This is achieved by applying the following gauge transformation [88]:

$$
a_{n}(t) \rightarrow \mathrm{e}^{-\mathrm{in}(K / \omega) \sin (\omega t)} a_{n}(t) .
$$

In this frame, and going to momentum space, the system of coupled Bogoliubov-de Gennes equations reduces to a $2 \times 2$ matrix [73], and the dynamics of the mode with momentum $q \in \mathrm{BZ}$ (first Brillouin zone) is described by [89] 
$i \partial_{t}\left(\begin{array}{l}u_{q} \\ v_{q}\end{array}\right)=\left(\begin{array}{cc}\varepsilon(q, t)+g & g \\ -g & -\varepsilon(-q, t)-g\end{array}\right)\left(\begin{array}{l}u_{q} \\ v_{q}\end{array}\right)$,

where

$$
\varepsilon(q, t)=4 J \sin \left(\frac{q}{2}\right) \sin \left(\frac{q}{2}+p_{0}-\frac{K}{\omega} \sin (\omega t)\right),
$$

with $p_{0}$ denoting the momentum of the initial BEC wave function. Throughout this paper, we set the lattice constant to unity $\left(a_{x}=1\right)$. Here, we introduced the parameter $g \equiv \rho U$, where $\rho=\left|a^{(0)}(0)\right|^{2}$ denotes the condensate density; the latter enters the normalization of the solution $a_{n}^{(0)}(t)$; the amplitudes $u_{q}, v_{q}$ of the Bogoliubov modes are defined by the expansion of the fluctuation term $\delta a_{n}$ in terms of Bloch waves

$$
\delta a_{n}(t)=\sum_{q} u_{q}(t) \mathrm{e}^{\mathrm{i} q \mathrm{n}}+v_{q}^{*}(t) \mathrm{e}^{-\mathrm{iqn}} .
$$

Equation (9) is numerically easier to integrate and will also constitute the starting point of the analytical analysis (see Sec. IV).

\section{NUMERICAL SIMULATION}

The general procedure outlined above can be implemented and solved numerically, regardless of the precise form of the physical band model. In more involved models, the initial state $a_{n}^{(0)}(0)$ can generically be determined by finding the ground state of the effective Hamiltonian $H_{\text {eff }}$, i.e., by solving the equivalent of Eq. (3) using imaginarytime propagation. The condensate wave function $a_{n}^{(0)}(t)$ is then determined by solving the time-dependent GPE with this initial condition, through real-time propagation (e.g., using a Crank-Nicolson integration scheme). Finally, the Bogoliubov equations are also solved over one driving period by real-time propagation, yielding the operator $\Phi(T)$, which is then exactly diagonalized (e.g., using a Lanczos algorithm). In the present case, this procedure can be shortcut by directly solving Eqs. (9), and we have checked that this produces the same results for all physical quantities. We now present the numerical results obtained for our model.

\section{A. Stability diagrams}

The stability diagram of the model in Eq. (2), which displays the behavior of the instability rate $\Gamma$ as a function of the interaction strength $g=U \rho$ and modulation amplitude $K / \omega$, is shown in Fig. 2, for a reasonably large driving frequency $\omega=5 \mathrm{~J}$. The stability boundary is similar to the one previously reported in Refs. [73,90]; in particular, the system is found to be stable in regions where $\mathcal{J}_{0}(K / \omega)=0$, which can be attributed to the fact that the dynamics is frozen by the cancellation of the effective tunneling [73]. At the transition to instability, the instability rate builds up continuously from zero and then increases when going further in the unstable regime.

Figure 3 shows similar stability diagrams for two other values of the driving frequency, $\omega=10 J$ and $\omega=J$. In the first case, we observe that the diagram is mostly unaffected by a change of $\omega$, provided $g$ is rescaled as $g \propto \omega^{2}$. More generally, except in what we refer to as the "low-frequency regime" (defined by the condition $\omega<4\left|J_{\text {eff }}\right|$ for the present model; see the next paragraph), transitions to instability always occur at some finite $g$, and the corresponding rate is found to mostly depend on the quantities $K / \omega$ and $g / \omega^{2}$ [91]. As we shall see in Sec. IV, this can be understood from the fact that the instability rate depends on a competition between $\omega$ and the Bogoliubov dispersion associated with the linearized effective GPE [i.e., the Bogoliubov dispersion stemming from the linear analysis of Eq. (3)],

$$
E_{\mathrm{av}}(q)=\sqrt{4\left|J_{\mathrm{eff}}\right| \sin ^{2}(q / 2)\left(4\left|J_{\mathrm{eff}}\right| \sin ^{2}(q / 2)+2 g\right)},
$$
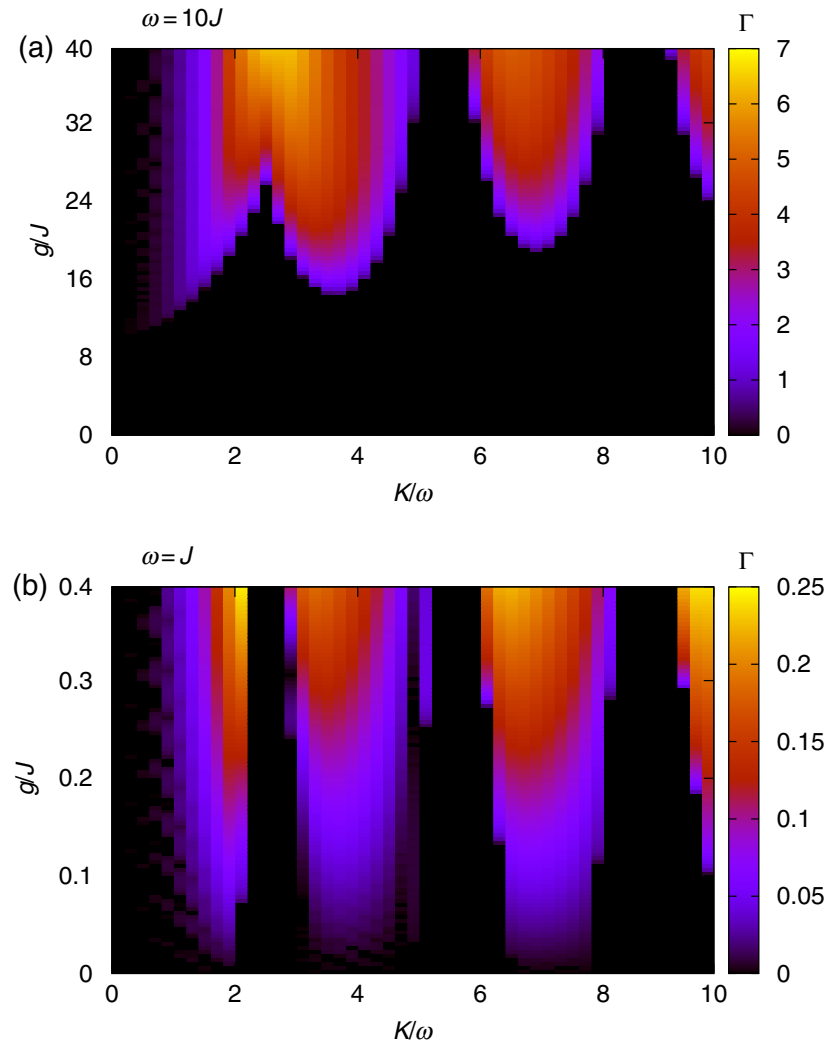

FIG. 3. Numerical instability rate $\Gamma$ as a function of interaction strength $g=U \rho$ and modulation amplitude $K / \omega$, for two values of the driving frequency $(\omega=10 J$ and $\omega=J)$. At large $\omega$ (i.e., outside the "low-frequency" regime, see text), the instability rates depend mostly only on $K / \omega$ and $g / \omega^{2}$, while in the lowfrequency regime, instabilities can occur at infinitesimal interaction strength. 
where the two cases $p_{0}=0, \pi$ are taken into account through the absolute value $\left|J_{\text {eff }}\right|$. As soon as the transition occurs at sufficiently large $g$, the term $4\left|J_{\text {eff }}\right| \sin ^{2} q / 2$ in this dispersion becomes negligible compared to $g$, which results in $E_{\mathrm{av}} \propto \sqrt{g}$, explaining the scaling indicated above.

This is no longer true in the "low-frequency regime" (see Fig. 3 for $\omega=J$ ), which we generically define through the criterion according to which $\omega$ is smaller than the effective free-particle bandwidth (i.e., $\omega<4\left|J_{\text {eff }}\right|$ for the model under consideration). In that case, we observe that instabilities may occur at any finite interaction strength $g$, which is related to the fact that $\omega$ is smaller than the bandwidth of the effective Bogoliubov dispersion (12) at $g=0$; see also the analytical analysis in Sec. IV for more details.

Finally, in the singular case where $J_{\text {eff }}=0$, the effective Bogoliubov dispersion $E_{\text {av }}(q)$ in Eq. (12) vanishes, and thus, the system is necessarily stable.

\section{B. Dynamics of physical observables}

Instead of solving the Bogoliubov equations [Eq. (5)] over one driving period only, we can also use these to compute the full time evolution of physical quantities, hence revealing both their long-time and micromotion dynamics. To do so, one has to choose an initial condition for the fluctuation term, $\delta a_{n}(t=0)$. In this section, we consider a generic (small) perturbation $\delta a_{n}(t=0)$, which has Fourier components over all Bogoliubov modes, i.e., of the generic form $\delta a_{n}(0)=\alpha \sum_{q} A_{q} \mathrm{e}^{\mathrm{iqn}}+B_{q} \mathrm{e}^{-\mathrm{iqn}}$, with $A_{q}$, $B_{q}$ being complex numbers and $\alpha$ a small amplitude. In Sec. VII, we discuss a more physical initial condition, in the framework of the periodically driven Bose-Hubbard model. Altogether, given such an initial condition, one can numerically evolve Eqs. (5) using real-time propagation, which yields $\delta a_{n}(t)$ [and thus also $a_{n}(t)=a_{n}^{(0)}(t)\left[1+\delta a_{n}(t)\right]$ in the linearized approximation], hence revealing the behavior of physical quantities.

\section{Energy growth and heating}

As an illustration, we first evaluate the energy in the rotating frame, which is given at time $t$ by

$$
\begin{aligned}
E(t)= & -J \sum_{n}\left[a_{n+1}(t) e^{\operatorname{in}(K / \omega) \sin (\omega t)}\right. \\
& \left.+a_{n-1}(t) e^{-\operatorname{in}(K / \omega) \sin (\omega t)}\right] a_{n}^{*}(t)+\frac{U}{2} \sum_{n}\left|a_{n}(t)\right|^{4} .
\end{aligned}
$$

In the linear approximation, one can explicitly recast this expression as a function of $\delta a_{n}$ and only keep terms of lowest order in $\delta a_{n}$. This yields $E(t)=E\left[a^{(0)}\right](t)+$ $E_{\text {fluct }}(t)$, where the energy of the fluctuations $E_{\text {fluct }}(t)$ is given to lowest order by

$$
\begin{aligned}
E_{\text {fluct }}(t) \approx & -J \sum_{n}\left[a_{n+1}^{(0)} \delta a_{n+1} e^{\operatorname{in}(K / \omega) \sin (\omega t)}\right. \\
& \left.+a_{n-1}^{(0)} \delta a_{n-1} e^{-\operatorname{in}(K / \omega) \sin (\omega t)}\right] a_{n}^{*(0)} \delta a_{n}^{*} \\
& +\frac{U}{2} \sum_{n}\left|a_{n}^{(0)}\right|^{4}\left[\delta a_{n}^{2}+\delta a_{n}^{* 2}+4\left|\delta a_{n}\right|^{2}\right] .
\end{aligned}
$$

The behavior of the energy of the fluctuations in Eq. (14) over several driving periods is shown in Fig. 4, in the stable and the unstable regimes (red curves), for an initial condition corresponding to $A_{q}=1$ and $B_{q}=0$. In the stable regime, it displays modulations due to micromotion but no long-term growth. Conversely, as anticipated for a parametric instability, the energy displays an exponential growth in the unstable regime. By fitting this growth, we find that its rate is given by $2 \Gamma$ [the factor 2 stems from the
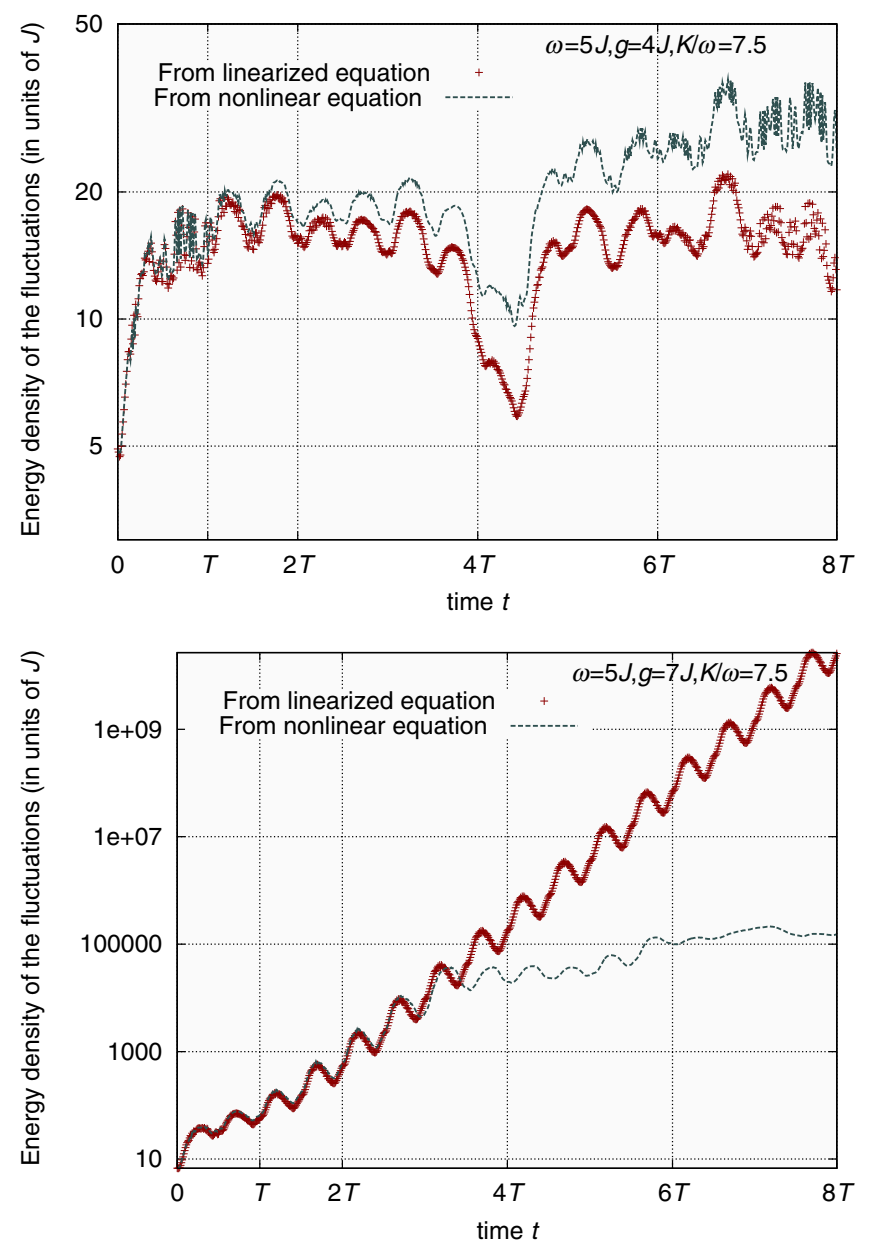

FIG. 4. Time evolution of the energy density of the fluctuations (in units of $J$ ) over eight driving periods for two values of $g$. In the stable regime (top panel, $g=4$ ), the energy displays variations due to micromotion but no long-term growth, while in the unstable regime (bottom panel, $g=7$ ), the energy curve features exponential growth, defining a heating rate compatible with the maximal Lyapunov exponent $\Gamma$ (see Fig. 5). The parameters are $A_{q}=1$ and $B_{q}=0$ (for the initial condition, see text), $\omega=5 \mathrm{~J}$, and $K / \omega=7.5$. 


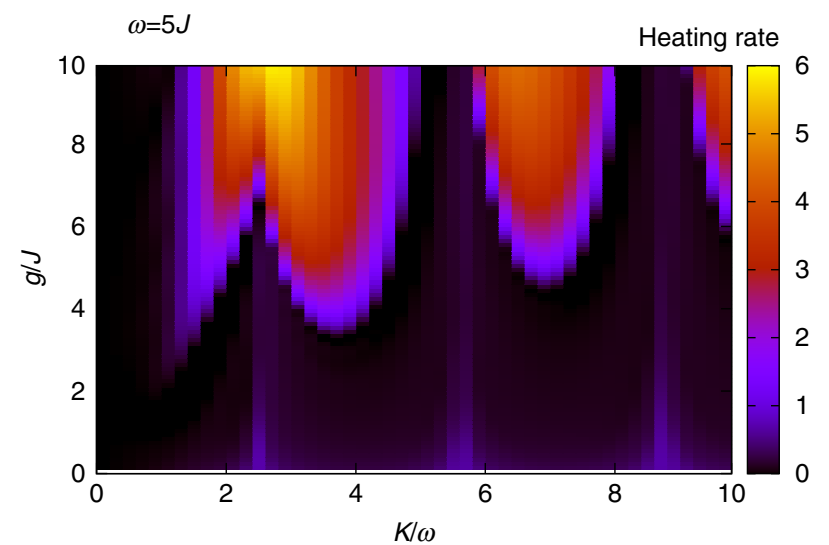

FIG. 5. Heating rate, obtained by fitting the exponential growth of the energy in Fig. 4. The resulting diagram is very similar to the instability diagram (Fig. 2), up to an overall numerical factor $\approx 2$ (see text).

square moduli $\left(\left|\delta a_{n}\right|^{2}, \ldots\right)$ in Eq. (14); see also below, Eq. (17)], which expresses the fact that the maximally unstable mode dominates the growth of the energy in the system. This unstable behavior, and the validity range of the related regime, will be further addressed in the next paragraphs. Figure 5 shows the diagram obtained by extracting the heating rates from energy curves and is found to be in excellent agreement with the stability diagram of Fig. 2. Therefore, we conclude that the instability rate $\Gamma$ can be used as a quantitative estimator of heating in such systems.

\section{Validity regimes of the calculation}

The computation of the full dynamics also highlights in which regimes our previous calculation of $\Gamma$ is expected to hold.

On the one hand, the calculation of the rate $\Gamma$ is based on a Floquet treatment of the Bogoliubov equations (5) and thus describes the dynamics at times

$$
t \gg T \text {. }
$$

In turn, the dynamics within one driving period, apparent in Fig. 4, cannot be captured by our analysis.

On the other hand, the analysis presented so far is based on the linear approximation of the GPE. To investigate nonlinear effects, we compared the time evolution obtained from the linearized Eq. (5) (red curves in Fig. 4, as previously discussed) with the time evolution of the original nonlinear GPE in Eq. (2) (grey dashed lines in Fig. 4), for the same initial condition. While the two graphs agree reasonably well in the stable regime, we find a significant deviation at longer times, within the unstable regime, because of the growth of the fluctuation term. This illustrates the intuitive fact that our linear-approximationbased treatment only holds at short times, imposing a second validity condition on our theory:

$$
t \ll t_{\text {lin }} .
$$

At longer times, nonlinear corrections to the evolution damp the exponential growth of the energy, which leads to a slowdown (saturation) of the heating dynamics.

Altogether, we find two natural time scales in the problem, and our approach thus requires the condition $T \ll t_{\text {lin }}$ to be relevant. Such a condition is fulfilled in a wide window of realistic system parameters.

\section{Instability regimes}

A third natural time scale arises when studying the growth of physical observables. In the linear treatment of the GPE, a generic physical observable can be expressed as

$$
O(t)=\sum_{q} \mathcal{O}\left[u_{q}(t), v_{q}(t)\right],
$$

where $\mathcal{O}\left[u_{q}(t), v_{q}(t)\right]$ is a functional, which is quadratic in the Bogoliubov modes $u_{q}(t)$ and $v_{q}(t)$; for instance, this is straightforward for the energy when substituting Eq. (11) into Eq. (14). This also applies to the noncondensed fraction when considering a weakly interacting BoseHubbard model in the mean-field interacting regime [see Eq. (45) in Sec. VII]. Using the fact that in our Floquet treatment of the Bogoliubov equation, each mode $q$ stroboscopically evolves according to the rate $s_{q}$, one can rewrite Eq. (15) as

$$
O\left(t_{N}\right)=\sum_{q} \mathcal{O}\left[u_{q}(0), v_{q}(0)\right] e^{2 s_{q} t_{N}},
$$

where the time $t_{N} \equiv N T$ denotes an integer multiple of the driving period $T$. By introducing $\Gamma=\max _{q} s_{q}$, as considered above, two cases may arise:

(a) If $\Gamma t_{N} \gg 1$, the growth of the maximally unstable mode dominates in Eq. (16), and we find that the observables stroboscopically grow exponentially with the rate $2 \Gamma$,

$$
O\left(t_{N}\right) \propto e^{2 \Gamma t_{N}},
$$

as already observed for the energy in Sec. III B 1 . Remarkably, this rate only involves the most unstable mode, and it is the same for all physical quantities. As announced above, this justifies the use of $\Gamma$ as a global instability rate in our theoretical analysis.

(b) If $\Gamma t_{N} \ll 1$, all exponentials in Eq. (16) can be linearized, yielding

$$
O\left(t_{N}\right)=O(0)+t_{N} \sum_{q} 2 s_{q} \mathcal{O}\left[u_{q}(0), v_{q}(0)\right]+\mathcal{O}\left(t_{N}^{2}\right) .
$$

In this regime, the energy increases linearly in time, with a rate given by the slope $2 \sum_{q} s_{q} \mathcal{O}\left[u_{q}(0), v_{q}(0)\right]$. The latter now involves a summation over all modes since no single mode contribution can be singled out at 


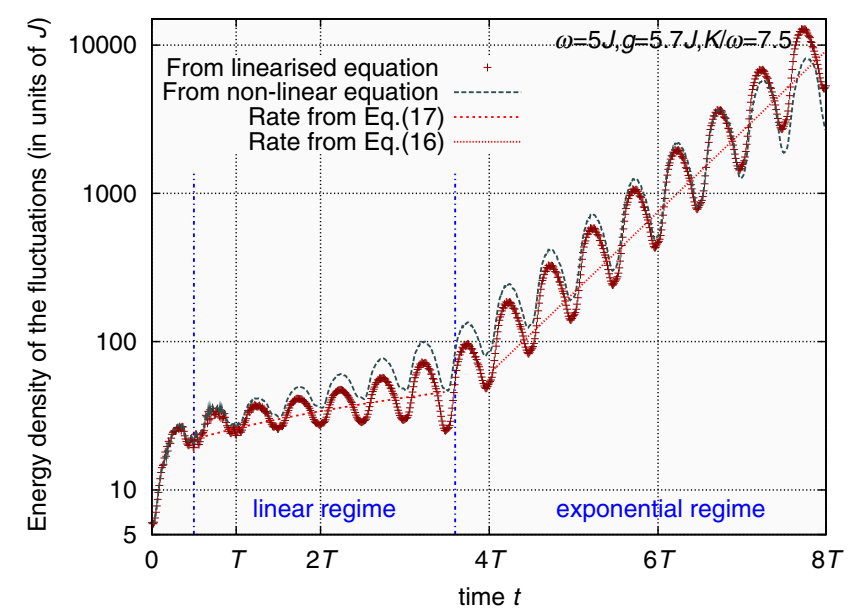

FIG. 6. Time evolution of the energy density of the fluctuations (in units of $J$ ) over eight driving periods for the initial conditions $A_{q}=1$ and $B_{q}=0$ (see text), for $\omega=5 J, K / \omega=7.5$, and $g=5.7$. In this regime, where $\Gamma$ is small, three regimes clearly show up: At very short times $t \ll T$, the initial growth is not described by our Floquet approach, as previously explained; at intermediate times $T \ll t \ll \Gamma^{-1}$, the growth is linear and described by Eq. (18) (red dotted line); at large times $t \gg \Gamma^{-1}$, the growth is exponential and described by Eq. (17) (red short-dotted line).

these early times $\left(t_{N} \ll 1 / \Gamma\right)$. Note also that, in this regime, the rate depends on the physical observable considered (through the functional $\mathcal{O}$ ).

For most values of the system parameters [such as those used in Fig. 4], ГT $\gg 1$, so the linear regime [Eq. (18)] is hidden in the first oscillation (which, as stated above, is not accurately captured by our Floquet approach). Yet, very close to the stability boundary, where $\Gamma$ is small, this marginal regime can become apparent in a very narrow range of parameters, as we illustrate in Fig. 6.
Here, three regimes clearly show up: At very short times $t \ll T$, the initial growth is not described by our Floquet approach, as previously explained; at intermediate times $T \ll t \ll \Gamma^{-1}$, the growth is linear and accurately described by Eq. (18); at longer times $t \gg \Gamma^{-1}$, the growth is exponential and well described by Eq. (17). Note that the linear regime is expected to be hard to probe in experiments since it appears at very short times, typically a few milliseconds (see also discussion in Sec. VIII).

\section{ANALYTICAL APPROACH}

\section{A. Linearized Gross-Pitaevskii equation as a parametric oscillator}

In momentum space, the Bogoliubov-de Gennes equations (9) constitute a set of uncoupled equations, which independently govern the time evolution of excitations with a given momentum $q$ : The mean-field analysis effectively reduces to a single-particle problem, and one can study how instabilities occur independently in each of those modes. The general mechanism underlying the appearance of parametric instabilities was suggested in Ref. [74], where a weakly interacting Bose-Hubbard model was investigated through a weak-coupling particle-numberconserving approximation; in that study, parametric resonances were shown to appear in the Bogoliubov equations, whenever the drive frequency was reduced below twice the single-particle bandwidth. A similar approach was considered in Ref. [92], which also provides an intuitive picture of the underlying mechanisms, in terms of the so-called Krein signature associated with the Bogoliubov modes.

The same phenomenon occurs in the present framework, as can be seen by performing a change of basis that recasts Eq. (9) into the following form (see Appendix $\mathrm{C}$ for details):

$$
i \partial_{t}\left(\begin{array}{c}
\tilde{u}_{q}^{\prime} \\
\tilde{v}_{q}^{\prime}
\end{array}\right)=\left[E_{\mathrm{av}}(q) \hat{\mathbf{1}}+\hat{W}_{q}(t)+\frac{g}{E_{\mathrm{av}}(q)}\left(\begin{array}{cc}
0 & h_{q}(t) \mathrm{e}^{-2 i E_{\mathrm{av}}(q) t} \\
-h_{q}(t) \mathrm{e}^{2 i E_{\mathrm{av}}(q) t} & 0
\end{array}\right)\right]\left(\begin{array}{c}
\tilde{u}_{q}^{\prime} \\
\tilde{v}_{q}^{\prime}
\end{array}\right) .
$$

Here, $E_{\mathrm{av}}(q)$ is the Bogoliubov dispersion associated with the effective (time-averaged) GPE, within the Bogoliubov approximation [see Eq. (12)]; $W_{q}(t)$ is a diagonal matrix of zero average over one driving period, which will play no role in the following (see Appendix $\mathrm{C}$ for its exact expression); and $h_{q}(t)$ is a (real-valued) function that can be Fourier expanded as

$$
\begin{aligned}
h_{q}(t) & =\frac{J}{2} 4 \sin ^{2}(q / 2) \sum_{l=-\infty}^{\infty}\left[\mathcal{J}_{l}(K / \omega)+\mathcal{J}_{-l}(K / \omega)\right] \mathrm{e}^{i l \omega t} \\
& =4 J \sin ^{2}(q / 2) \sum_{l=-\infty}^{\infty} \mathcal{J}_{2 l}(K / \omega) \mathrm{e}^{i 2 l \omega t}
\end{aligned}
$$

with $\mathcal{J}_{l}(z)$ the $l$ th Bessel function of the first kind. Importantly, the specific form of Eq. (19) allows one to clearly distinguish between the contribution due to the time-averaged dynamics, as captured by the effective dispersion $E_{\mathrm{av}}(q)$, and the contribution due to the fulltime evolution $\left[W_{q}(t), h_{q}(t)\right]$. As will be shown below, the full-time dependence of the system is crucial for understanding the origin of its instabilities, through the properties of the real-valued function $h_{q}(t)$.

Casting the equations of motion in the form (19) allows one to directly identify any parametric resonance effects. To see this, we recall the reasoning of Ref. [74], which was based on applying a rotating-wave approximation (RWA) 
to Eq. (19). This approach amounts to neglecting all fastoscillating terms in Eq. (19), which is equivalent to performing a time average over a drive period $T$; we note that this treatment captures the stroboscopic dynamics ( $t=T \times$ integer), which is consistent with the definition of the instability rates, as extracted from Lyapunov exponents in Sec. II. If one disregards the expression in Eq. (20) for now, and if one naively assumes that the lowest frequency appearing in $h_{q}(t)$ in Eq. (19) is $\omega$, then a dominant contribution to the dynamics is expected when the resonance condition $\omega=2 E_{\mathrm{av}}(q)$ is fulfilled, resulting in the time-independent nondiagonal term in the matrix displayed in Eq. (19). Keeping this resonant term only yields a time-independent $2 \times 2$ matrix that can be diagonalized and whose eigenvalues (i.e., the Lyapunov exponents) are found to exhibit an imaginary part, yielding a nonzero instability rate. This argument was invoked in Ref. [74] to justify the intuitive stability criterion $\omega>2 W_{\text {eff }}$ [with $W_{\text {eff }}=\sqrt{4\left|J_{\text {eff }}\right|\left(4\left|J_{\text {eff }}\right|+2 g\right)}$ the bandwidth of the effective Bogoliubov dispersion], which states that instability arises from the absorption of the energy $\omega$ to create a pair of Bogoliubov excitations on top of the condensate. However, a more careful examination shows that the instability rates inferred from this idea are not consistent with our numerical simulations. Moreover, to get a sense of the additional dilemma one is faced with, we note that the expression for $h_{q}(t)$ in Eq. (20) actually only contains even harmonics of the modulation, and therefore, following the reasoning above, the resonance condition should then read $2 \omega=2 E_{\mathrm{av}}(q)$. Yet, such a criterion provides a wrong estimate of the stability-instability boundaries. Hence, it appears that this simple explanation needs to be thoughtfully revised.

In the following, we present a more rigorous analytical evaluation of the instability rates for our system. Similar to Ref. [74], our derivation relies on the fact that the Bogoliubov equations (19) precisely take the form of a socalled parametric oscillator. In Appendix B, we briefly recall some useful results about this paradigmatic model [3], which describes a harmonic oscillator of eigenfrequency $\omega_{0}$ driven by a weak sinusoidal perturbation of frequency $\omega$ and amplitude $\alpha$. In brief, such a model displays a parametric instability for $\omega \approx 2 \omega_{0}$. Importantly, the instability is maximal when this resonance condition is fulfilled, but it occurs in a whole range of parameters around this point. As detailed in Landau and Lifshits [3], the width of the resonance domain and the instability rates in the vicinity of the resonance can be calculated perturbatively by introducing the detuning $\delta=\omega-2 \omega_{0}$ and solving the equations perturbatively in $\delta$ and amplitude $\alpha$ (see Appendix B).

More specifically, the Bogoliubov equations (19) exactly take the form of the parametric oscillator [Eq. (B2)], with the frequency $\omega_{0}$ of the unperturbed oscillator being identified with the dispersion $E_{\mathrm{av}}(q)$. Thus, it immediately follows that the model is equivalent to a set of independent parametric oscillators, one for each mode $q$. Two points should be emphasized here: First, all of those parametric oscillators depend on $q$ in a different way and will thus exhibit different resonance conditions; second, the function $h_{q}(t)$ in Eq. (19) is not a pure sinusoid as in Eq. (B2), but rather, it contains all (even) harmonics of the driving frequency $\omega$. Altogether, given those two remarks, resonances are expected as soon as one of the harmonics of the modulation, of energy $l \omega$, is close to twice the energy of
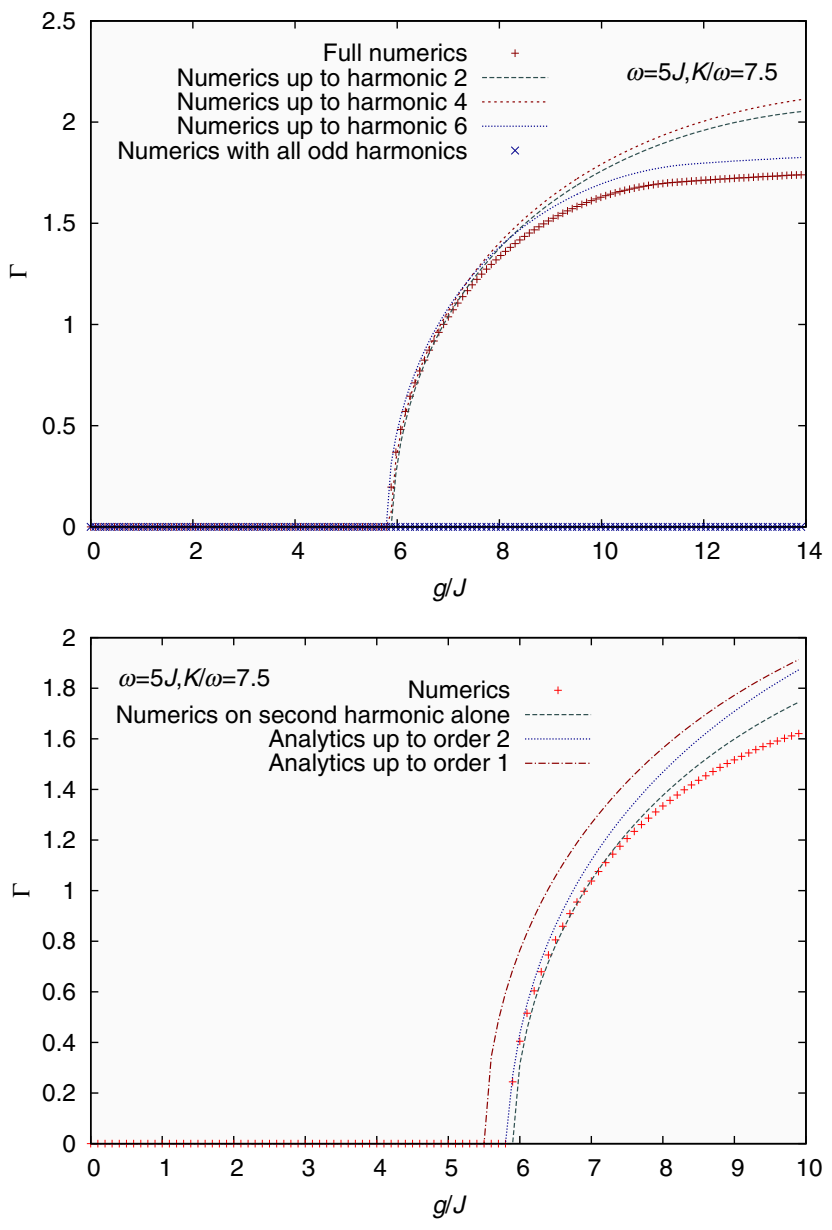

FIG. 7. Top panel: Instability rate $\Gamma$ as a function of the interaction strength $g$ for $\omega=5 J$ and $K / \omega=7.5$, numerically computed from the Bogoliubov equations (cf. Sec. II), taking into account only a few harmonics of the modulation in the function $h_{q}(t)$. As theoretically predicted from the expression of $h_{q}(t)$ [Eq. (20)], the odd harmonics do not contribute, while the first even ones successfully describe the full numerical calculation. Bottom panel: Comparing the analytical and numerical results for the instability rate $\Gamma(g)$, using the same parameters as above. The red crosses correspond to the full numerical solution, while the blue dashed lines are numerically obtained by only taking the second harmonic of the drive into account. As generically observed in the A zones of the stability diagram (Fig. 2), the numerical instability rates are well captured by the perturbative analytical approach, implemented here up to order 1 (red dotteddashed line) and 2 (blue dotted line). 
any of the (effective, time-averaged) Bogoliubov modes, $2 E_{\mathrm{av}}(q)$.

Before digging into more technical details, let us acquire some intuition about the general mechanisms behind this phenomenon. To do so, consider the stability diagram in Fig. 2 (i.e., outside of the low-frequency regime [93]). In this case, in the lowest part of the stability diagram (small $g$ ), $\omega$ is generically large compared to the bandwidth of the effective dispersion $E_{\mathrm{av}}(q)$, so no resonance can occur. When increasing $g$ at fixed $K / \omega$, the first mode to exhibit a resonance will be that of maximal $E_{\mathrm{av}}(q)$, i.e., $q=\pi$. Naively, the first resonance would be due to the first harmonic $l=1$ and would occur for $2 E_{\mathrm{av}}(q=\pi)=\omega$, which was also the argument in Ref. [74]. However, as already discussed, the function $h_{q}(t)$ only contains even harmonics, and the first resonance to occur is, therefore, the one corresponding to $l=2$. At first sight, this might seem to be in contradiction with the intuitive stability criterion $2 E_{\text {av }}(\pi)=\omega$ since the $l=2$ resonance is centered around $E_{\mathrm{av}}(\pi)=\omega$; however, as we shall discuss in more detail below, the key point is that the resonance domain has a finite width: Although centered around $E_{\mathrm{av}}(\pi)=\omega$, its boundaries are in fact close to the point where $2 E_{\text {av }}(\pi)=\omega$. Resonances due to higher harmonics (fourth, sixth, etc.) become important only for higher values of $g$, and we can, to a first approximation, restrict the analysis to the second harmonic only.

To confirm this intuition, we have verified that restricting our analysis to the second harmonic only is generally sufficient to estimate the stability boundary and to recover the instability rate in its vicinity [94]. Deeper in the unstable region, the fourth and, eventually, the sixth harmonics are progressively required to recover the agreement with the numerical results (see Fig. 7).

\section{B. Effective model}

To simplify the calculations, we restrict our analysis to the second harmonic (see discussion above); we stress that higher-order resonances can be treated along the same lines. The equations of motion [Eq. (19)] then read

$$
i \partial_{t}\left(\begin{array}{c}
\tilde{u}_{q}^{\prime} \\
\tilde{v}_{q}^{\prime}
\end{array}\right)=\left[E_{\mathrm{av}}(q) \hat{\mathbf{1}}+\hat{W}_{q}(t)+\frac{\alpha_{q} E_{\mathrm{av}}(q)}{2}\left(\begin{array}{cc}
0 & \cos (2 \omega t) \mathrm{e}^{-2 i E_{\mathrm{av}}(q) t} \\
-\cos (2 \omega t) \mathrm{e}^{2 i E_{\mathrm{av}}(q) t} & 0
\end{array}\right)\right]\left(\begin{array}{c}
\tilde{u}_{q}^{\prime} \\
\tilde{v}_{q}^{\prime}
\end{array}\right),
$$

with

$$
\alpha_{q}=16 J \mathcal{J}_{2}(K / \omega) \sin ^{2}(q / 2) \frac{g}{\left[E_{\mathrm{av}}(q)\right]^{2}} .
$$

Therefore, for a fixed $q$, these equations are now strictly equivalent to the equations of motion for the parametric oscillator of Eq. (B2), with the following identifications:

$$
\begin{aligned}
\omega_{0} & \rightarrow E_{\mathrm{av}}(q), \\
\omega & \rightarrow 2 \omega, \\
\alpha & \rightarrow \alpha_{q} .
\end{aligned}
$$

As a result, each mode $q$ exhibits a resonance domain centered around $E_{\mathrm{av}}(q)=\omega$. The instability rate is generically maximal around the resonance point (defining the maximal rate $s_{q}^{\max }$ ), and it decreases until it cancels at the edges of the resonance domain. In order to obtain analytical estimates of the instability rate and the width of the resonance domain, one can apply the procedure detailed in Appendix B, which amounts to solving the problem perturbatively in the detuning $\delta=2 \omega-2 E_{\mathrm{av}}(q)$ and amplitude $\alpha_{q}$. To zeroth order, the instability only arises when the resonance condition is precisely fulfilled, which defines the rate "on resonance," $s_{q}^{*}$, which is given by

$$
s_{q}^{*}=g \frac{4 J \mathcal{J}_{2}(K / \omega) \sin ^{2}(q / 2)}{E_{\mathrm{av}}(q)} .
$$

To first order, the instability rate reads [see Eq. (B3) with the substitutions (22)]

$$
s_{q}=s_{q}^{*} \sqrt{1-\left(\frac{\left[\omega-E_{\mathrm{av}}(q)\right] E_{\mathrm{av}}(q)}{4 J \mathcal{J}_{2}(K / \omega) \sin ^{2}(q / 2) g}\right)^{2}},
$$

whenever the argument in the square root is positive, and $s_{q}=0$ otherwise. The associated instability rate $s_{q}$ is thus maximal on resonance (hence, $s_{q}^{\max }=s_{q}^{*}$ ), and it decreases when going towards the boundaries of the resonance domain. At second order, $s_{q}$ is given by the solution of the implicit equation (B4) with the substitutions (22), which can be found numerically; this can be performed to improve the accuracy of the analytical rate's value. In particular, at this order, we note that the actual maximal instability point is then slightly shifted from the resonance point (i.e., $s_{q}^{\max } \neq s_{q}^{*}$ ). Altogether, the total instability rate is given by [Eq. (7)]

$$
\Gamma=\max _{q} s_{q} .
$$

As a function of $g$ [which enters $E_{\mathrm{av}}(q)$; see Eq. (12)], the instability rate of a given mode $q$ forms a "bumped curve" 


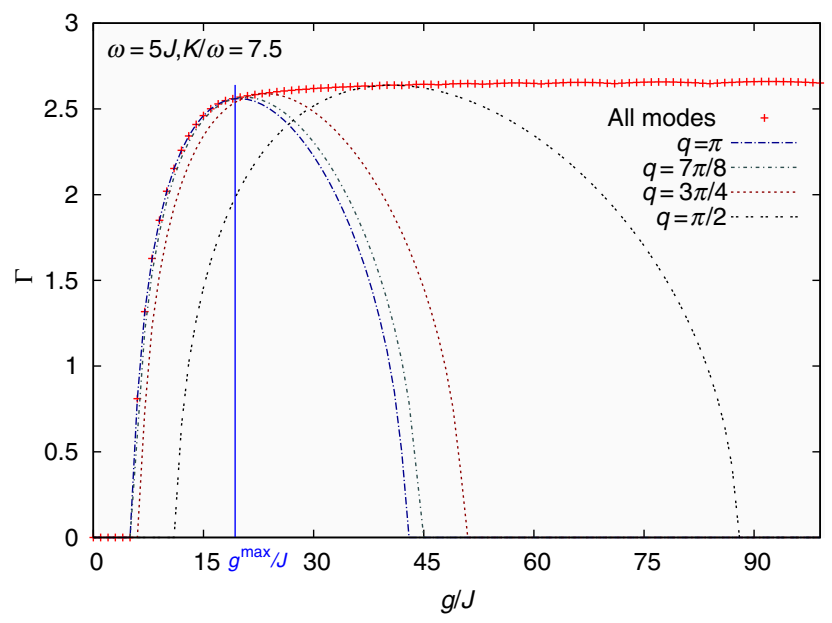

FIG. 8. Total instability rate $\Gamma$ (red crosses) and instability rates $s_{q}$ of a few individual modes (dashed lines), numerically computed as a function of interaction strength $g$ for $\omega=5 \mathrm{~J}$ and $K / \omega=7.5$. The instability rate associated with an individual mode $q$ is maximal for $E_{\mathrm{av}}(q)=\omega$ and decreases around it. The total instability rate is given by the envelope of all those curves.

(see Fig. 8), which to a first approximation simply follows from Eq. (24). Since the resonance domain for each mode is centered around a different energy (and therefore a different $g$ ), the curves corresponding to various modes are slightly shifted, and the total instability rate is given by the envelope of all those curves (see Fig. 8).

In particular, the curve centered around the smallest values of $g$ (i.e., the leftmost curve in Fig. 8, near the transition point) is the one associated with the mode of highest $E_{\mathrm{av}}$, namely, $q=\pi$. Let us denote by $g^{\max }$ the value of $g$ where this curve is maximal: At first order, this corresponds to the solution of the resonance condition $\omega=E_{\text {av }}(\pi)=\sqrt{4\left|J_{\text {eff }}\right|\left(4\left|J_{\text {eff }}\right|+2 g\right)}$. Then, we identify two cases:

(a) For $g>g^{\max }$, there always exists, in the thermodynamic limit, a single mode $q$ that is maximally unstable, and the total instability rate, Eq. (25), is thus given by the rate of this particular mode. At first order (see discussion above), this mode is the one fulfilling the resonance condition $\omega=E_{\mathrm{av}}(q)$, and the instability rate is given by the rate on resonance of this particular mode $q_{\text {res }}$, so

$$
\Gamma=s_{q_{\mathrm{res}}}^{*}=\left|\frac{\mathcal{J}_{2}(K / \omega)}{\mathcal{J}_{0}(K / \omega)}\right| \frac{g}{\omega}\left(\sqrt{g^{2}+\omega^{2}}-g\right),
$$

where we have explicitly reexpressed $q_{\text {res }}$ using the expression $\omega=E_{\mathrm{av}}\left(q_{\mathrm{res}}\right)$.

(b) Conversely, for $g<g^{\max }$, the instability rate is only governed by the mode $q=\pi$ (see Fig. 8), and it cannot be estimated from the knowledge of the associated rate on resonance. Therefore, to capture the behavior of the instability rate near the transition point, as well as the stability boundary itself, one can indeed restrict our analysis to the mode $q=\pi$, but one in turn has to resort to the perturbative expansion around the resonance point discussed in Appendix B. In other words, in that case, one has

$$
\Gamma=s_{\pi},
$$

where $s_{\pi}$ is given at lowest order by Eq. (24) with $q=\pi$. The stability boundary can also be computed at any required order following the procedure indicated in Appendix B, which yields, up to second order,

$$
\omega=E_{\mathrm{av}}(\pi)+\frac{4 J \mathcal{J}_{2}(K / \omega) g}{E_{\mathrm{av}}(\pi)}-\frac{4 J^{2} \mathcal{J}_{2}^{2}(K / \omega) g^{2}}{E_{\mathrm{av}}(\pi)^{3}}+\mathcal{O}\left(g^{3}\right) .
$$

Interestingly, in the high-frequency regime, since the transition occurs at sufficiently large $g$, so that $E_{\text {av }} \propto \sqrt{g}$, one recovers that the boundary is a function of the combination $g / \omega^{2}$, as previously observed numerically in Sec. III.

When decreasing the frequency, the transition occurs at lower and lower values of $g$ : Since all corrections of any order tend to vanish at small $g$ in Eq. (28), the transition point at vanishing $g$ is simply obtained for $\omega=4 J_{\text {eff }}$, recovering the criterion for entering the low-frequency regime previously discussed. In this regime, which in some sense corresponds to a negative $g^{\max }$, one is always in case (a) and the rate $\Gamma$ is given by Eq. (26).

Altogether, to capture the behavior of the instability rate in the whole range of parameters, one should use Eqs. (24) and (25), which include all modes as well as the first correction due to the finite detuning. Note that the perturbative expansion yielding those expressions is expected to hold, provided $\alpha_{q}$ is not too large, i.e., $E_{\text {av }}(q)$ is not too small (see below for a discussion of the breaking points of the approach).

\section{Results based on the analytical approach}

We now show the results obtained from this analytical approach for the instability rate, Eq. (25), with $s_{q}$ being generically computed to second order in the detuning (unless explicitly specified). The stability diagram is very similar to the one obtained numerically (see Fig. 2). Let us investigate in more detail this agreement and comment on the small differences between the numerical and analytical diagrams.

\section{Agreement regions}

For values of $K / \omega$ that are not too close to the zeros of the Bessel functions $\mathcal{J}_{0}$ and $\mathcal{J}_{2}$ [95] (i.e., not too close to the edges of the lobes in the stability diagram, zones A in Fig. 2), the analytical calculation gives a very good estimate 
of both the transition point and the instability rate, up to moderate interactions $g$ (Fig. 7). At higher $g$, more harmonics become important (presumably, in a complex and "coupled" way; we verified that building independent effective models for separate harmonics does not reproduce the numerics accurately). Zones where $\mathcal{J}_{4}$ vanishes are "favorable" since the first correction to the effective model [Eq. (21)] is absent, and the agreement between analytics and numerics survives for larger values of $g$.

In the vicinity of the "common" zeros of $\mathcal{J}_{2}(K / \omega)$ and $\mathcal{J}_{0}(K / \omega)$ (i.e., "between" the lobes of the stability diagram, zones B in Fig. 2), both the analytics and numerics agree and predict a stable regime.

\section{Disagreement regions}

A detailed description of the small disagreement regions is provided in Appendix D. In brief, the analytics break down into two main regions: (i) Around the first zero of $\mathcal{J}_{0}$ (i.e., the only zero of $\mathcal{J}_{0}$ where $\mathcal{J}_{2}$ is significant; zones $\mathrm{C}$ on Fig. 2), the perturbative approach breaks down because of the vanishing of the effective dispersion $E_{\mathrm{av}}$, and the analytical approach fails. (ii) Near the common zeros of $\mathcal{J}_{0}$ and $\mathcal{J}_{2}$ (i.e., "between" the lobes of the stability diagram), we find that the numerics and the analytics disagree in the way the stable zones close at large $g$. We identified two main causes for this effect: the breakdown of the perturbative approach due to the vanishing of $E_{\mathrm{av}}$, and the vanishing of the second harmonics near the zeros of $\mathcal{J}_{2}$. The consequences of these factors taken together are very involved since they can both compensate for each other and compete. In brief, at the left border of each lobe (zones D in Fig. 2), the numerics displays a transition to instability (although showing up only at large $g$ ), which is not captured by the analytics. At the right border of the lobes (zones E), the instability predicted by the full numerics is most likely due to a joint effect of the second and higher-order harmonics and is therefore not accurately captured by the analytical approach [see Fig. 21(b)].

Nevertheless, the disagreement regions constitute very narrow zones in the stability diagram. Figure 2 summarizes the zones where the analytical and numerical calculations agree (in green) and the ones where they disagree qualitatively (in red) or just quantitatively (in orange).

\section{Scaling in limiting cases}

The analytical solutions provide a general scaling behavior for the instability rates as a function of the model parameters, which we analyze for three limiting cases.

(a) Weak interactions: If $\omega$ is larger than the effective free-particle bandwidth of the model (high-frequency regime), the system is stable at $g \approx 0$, and it features a transition to an unstable phase at some finite $g_{c}$, with a scaling [see Eq. (24)]

$$
\Gamma \propto \sqrt{\left(g-g_{c}\right)} .
$$

Conversely, if $\omega$ is smaller than the effective freeparticle bandwidth (low-frequency regime), the system is typically unstable at very small $g$, and [see Eq. (26)]

$$
\Gamma \propto g
$$

(b) Weak driving amplitudes: The analysis of the transition to the unstable phase from $K=0$ (where the system is stable) to finite $K$ is subtle since this limit does not commute with the thermodynamic limit. Indeed, one has to note that the resonance domain of each mode has a vanishing width when $K \rightarrow 0$. Therefore, for any finite-size system, instabilities at vanishing $K$ occur only for discrete values of the parameters (fulfilling one of the resonance conditions associated with a specific mode); it is only when increasing $K$ that those instability regions grow in parameter space, eventually merging and forming the first lobe of the stability diagram. In the thermodynamic limit, one nevertheless finds the general scaling

$$
\Gamma \propto K^{2}
$$

which stems from the second-order Bessel function, which dominates at low $K$ in Eq. (26).

(c) Low driving frequency: In this limit, one finds [see Eq. (26)]

$$
\Gamma \propto \omega \times f(K / \omega),
$$

where $f$ is a generic function of $K / \omega$ only.

\section{FINITE-SIZE SYSTEMS: FROM A DOUBLE WELL TO THE ENTIRE LATTICE}

The analytical approach, which treats different modes independently, allows one to readily predict the behavior of finite-size systems under periodic modulation, close to a parametric resonance. In this case, the "continuous" dispersion $E_{\text {av }}(q)$ is replaced by discrete energy modes. For a small number of lattice sites (and thus modes), the total instability rate $\Gamma=\max _{q} s_{q}$, which is given by the envelope of the curves associated with individual modes (see Fig. 8), may not be a monotonic curve as a function of $g$. Instead, it is rather composed of disjointed bumps if the resonance domains of two consecutive modes are disconnected. Figure 9 shows how individual modes contribute to the total growth rate when increasing the number of sites, both inside and outside the low-frequency regime. Interestingly, the situation is slightly different in these two cases. Outside the low-frequency regime, i.e., for $\omega \gtrsim J_{\text {eff }}$, the onset of instability is governed by the mode $q=\pi$ 
(see Sec. IV), and therefore, the critical frequency, below which the system becomes unstable, happens to be the same for both an infinite and a two-site system. Since the mode $q=\pi$ remains the "most unstable" one, all the way up to large values of $g$ (see Fig. 8), the stability diagram of a two-site system is in fact very similar to the one associated with an infinite system (whereas a one-site system is trivially stable) [96]. Conversely, in the low-frequency regime $\left(\omega \lesssim J_{\text {eff }}\right)$, instability occurs already at vanishingly small $g$, induced by a certain resonant mode $q_{\text {res }}$ fulfilling
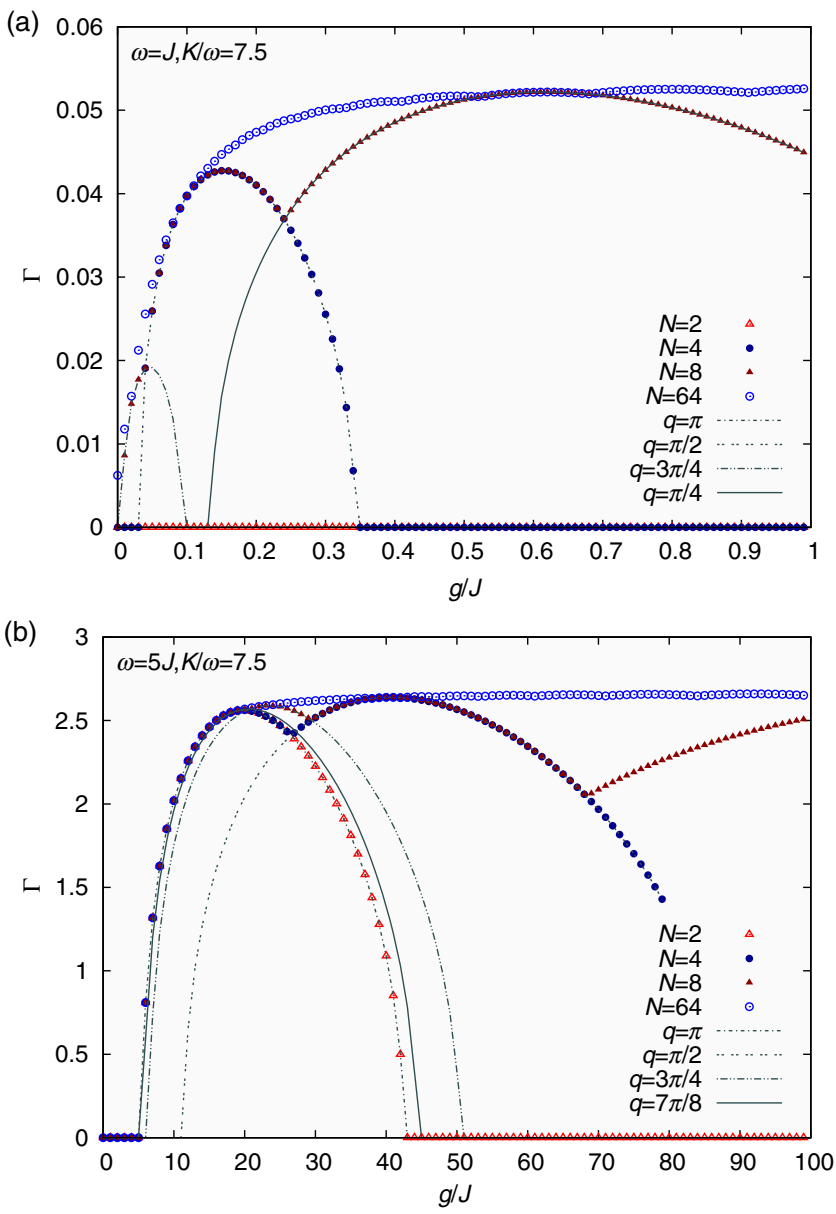

FIG. 9. Total instability rate and contributions of a few modes as a function of the interaction strength $g$, for $\omega=J$ (lowfrequency regime) and $\omega=5 J$ (outside the low-frequency regime), and for an increasing number of lattice sites $N=2$, $4,6,64$. The total instability rate is the envelope of the curves associated with "available" modes in the system (for $N=2$, only $q=0, \pi$; for $N=4$, only $q=0, \pi / 2, \pi, 3 \pi / 2 \ldots)$. The curve gets smoother when increasing the number of sites. Outside the low-frequency regime, the transition to instability is governed by the mode $\pi$ and already captured by a two-site system. In the low-frequency regime, the instability rate at low $g$, which is governed by a certain mode $q_{\text {res }}$, is probed with more and more accuracy when gradually increasing $N$ : Here, the two-site system is always stable since $q_{\text {res }}>\pi$, the four-site system displays a transition since $q_{\text {res }}>\pi / 2$, and then this transition lowers when probing $q_{\text {res }}$. the resonance condition (see Sec. IV). Increasing the number of sites therefore lowers the instability boundary as a function of $g$ since increasing the number of points in momentum space allows for the states to get closer to the maximally unstable mode. Moreover, if the number of sites $N$ is too small, the discretization in momentum space is so rough that the resonance domains of consecutive modes may be disconnected, resulting in "islands" of instability in the phase diagram. These islands begin to merge with increasing $N$, as the resonance domains of adjacent modes overlap, only gradually approaching the phase diagram of an infinite system. Both our analytical and numerical methods capture this behavior and agree for any number of lattice sites. Interestingly, since the instability rate is determined by its maximal value over all modes (i.e., the envelope of all curves in Fig. 9), its estimate obtained for finite-size systems is already very decent, and further increasing the number of sites just smoothens the curve of the instability rate as a function of $g$.

We point out that for very small systems, there might be minor corrections due to edge states: Indeed, the previous treatment, formulated in momentum space, tacitly implies periodic boundary conditions. However, in the lab frame, the drive actually breaks translational invariance, and the boundary conditions are intrinsically open, possibly yielding different selection rules for states near the edges. Although such a difference is expected to play a negligible role in large systems, it may lead to minor but noticeable deviations in small systems.

\section{EFFECT OF TRANSVERSE DIRECTIONS: 2D LATTICES VS TUBES}

So far, our study focused on the origin of parametric instabilities that occur in a driven system, which satisfies the 1D GPE on a lattice (2). As further discussed in Sec. VII, this model can be used to describe the physics of weakly interacting bosonic atoms, trapped in a 1D optical lattice. However, from an experimental point of view, it is intriguing to determine the effects of transverse directions on the stability diagram and instability rates. Indeed, optical-lattice experiments involving weakly interacting bosons [39,41] typically feature continuous transverse degrees of freedom, commonly referred to as "tubes" or "pancakes." In addition, experiments realizing artificial magnetic fields [97,98] involve two-dimensional optical lattices, and hence, it is relevant to study the fate of instabilities as one transforms a 1D optical lattice into a full 2D lattice, by adding sites (and allowing for hopping processes) along a transverse direction.

In this section, we extend our previous analysis to study the effects of (i) a secondary tight-binding-lattice direction (resulting in a ladder or a full 2D lattice) and (ii) an additional continuous ("tube") degree of freedom. In the following, we assume that the periodic modulation remains 
exclusively aligned along the original tight-binding lattice dimension.

\section{A. Two-dimensional lattice geometry}

In this section, we consider the addition of a transverse lattice direction, aligned along the $y$ axis; by doing so, we keep the same time-dependent modulation as before, which is hence exclusively aligned along the $x$ axis. Our aim is to study how an increase in the number of sites along the transverse direction affects the instability rates, previously evaluated for the 1D configuration. Theoretical studies $[64,66]$ have reported that heating and collisional processes are enhanced by the presence of a transverse direction, which provides crucial information for current experimental studies.

For this extended model, the time-dependent GPE reads

$$
\begin{aligned}
i \partial_{t} a_{m, n}= & -J\left(a_{m, n+1}+a_{m, n-1}+a_{m+1, n}+a_{m-1, n}\right) \\
& +K \cos (\omega t) m a_{m, n}+U\left|a_{m, n}\right|^{2} a_{m, n},
\end{aligned}
$$

where each site of the underlying (2D) square lattice is now labeled by two integers $(m, n)$. As for the 1D case (Sec. II), the condensate wave function at time $t=0$ is again given by a Bloch state $\mathrm{e}^{i\left(p_{x} m+p_{y} n\right)}$, with momentum $p_{x}=0$ if $\mathcal{J}_{0}(K / \omega)>0$ and $p_{x}=\pi$ if $\mathcal{J}_{0}(K / \omega)<0$; for this choice of the time modulation, the momentum along the $y$ direction is necessarily $p_{y}=0$. The time-dependent Bogoliubov-de Gennes equations, which govern the time evolution of the mode $\mathbf{q}=\left(q_{x}, q_{y}\right)$, take the form

$i \partial_{t}\left(\begin{array}{l}u_{q} \\ v_{q}\end{array}\right)=\left(\begin{array}{cc}\varepsilon(\mathbf{q}, t)+g & g \\ -g & -\varepsilon(-\mathbf{q}, t)-g\end{array}\right)\left(\begin{array}{l}u_{q} \\ v_{q}\end{array}\right)$,

where

$\varepsilon(\mathbf{q}, t)=4 J \sin \frac{q_{x}}{2} \sin \left(\frac{q_{x}}{2}+p_{x}-\frac{K}{\omega} \sin \omega t\right)+4 J \sin ^{2} \frac{q_{y}}{2}$,

which is a straightforward generalization of Eq. (9). Therefore, both the numerical and the analytical methods presented in the previous sections can be readily applied to Eq. (30).

Figures 10 and 11 show the stability diagrams, obtained numerically by increasing the number $N_{y}$ of transverse lattice sites (from 4 to 16); they correspond to the drive frequency $\omega=10 J$ (i.e., high-frequency regime, where $\omega$ is greater than the effective bandwidth) and $\omega=5 J$ (i.e., low-frequency regime), respectively. Since the motional degrees of freedom along the $x$ and $y$ directions are independent of each other, the problem being separable, the situation is analogous to the one previously discussed in the case of finite-size systems.
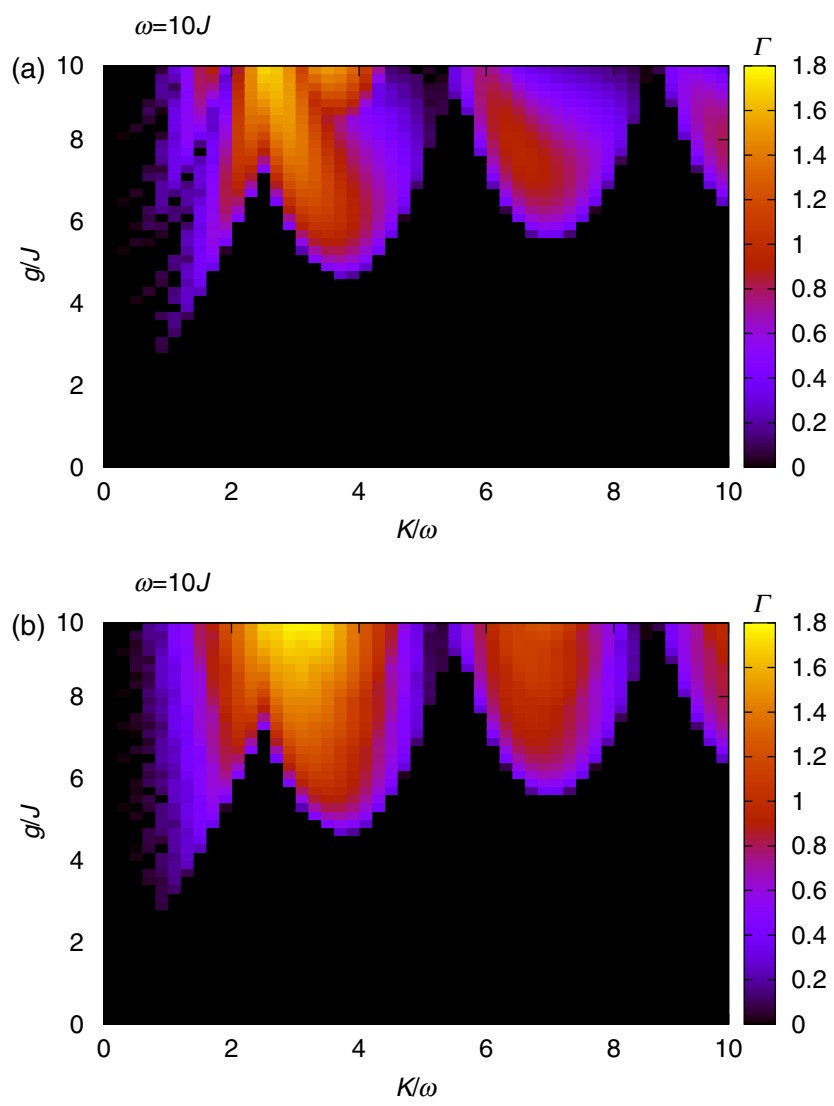

FIG. 10. Numerical instability rate as a function of interaction strength $g=U \rho$ and modulation amplitude $K / \omega$, for $\omega=10 \mathrm{~J}$ (i.e., outside the low-frequency regime) and for two different numbers of lattice sites in the transverse direction: $N_{y}=4$ (top panel) and $N_{y}=16$ (bottom panel).

Let us summarize the results: Away from the lowfrequency regime (Fig. 10), namely, for $\omega>4\left(J+J_{\text {eff }}\right)$ [the drive frequency is again compared to the modified (effective) free-particle bandwidth], the system is stable for small $g$, and the onset of instability is governed by the mode of maximal energy (i.e., the first mode to exhibit a resonance), which now corresponds to $\mathbf{q}=(\pi, \pi)$. Therefore, as soon as two sites are present in the transverse direction, the transition point to the unstable regime is well captured. Moreover, both the instability boundary and the instability rates in its vicinity are expected to remain unaffected when increasing $N_{y}$ since they are dominated by the mode $(\pi, \pi)$ [see Eq. (27)]. Conversely, in the lowfrequency regime, there is an instability at vanishingly small $g$, which is driven by a certain mode $\left(q_{x}, q_{y}\right)$ : In this case, adding the number of transverse sites lowers the instability boundary, as increasing the resolution in momentum space allows one to get closer to this maximally unstable mode.

Using Eq. (30), one can readily extend the analytical approach of Sec. IV. Indeed, all the results of Sec. IV remain applicable in the present case, provided that the dispersion $E_{\mathrm{av}}(\mathbf{q})$ is now replaced by 

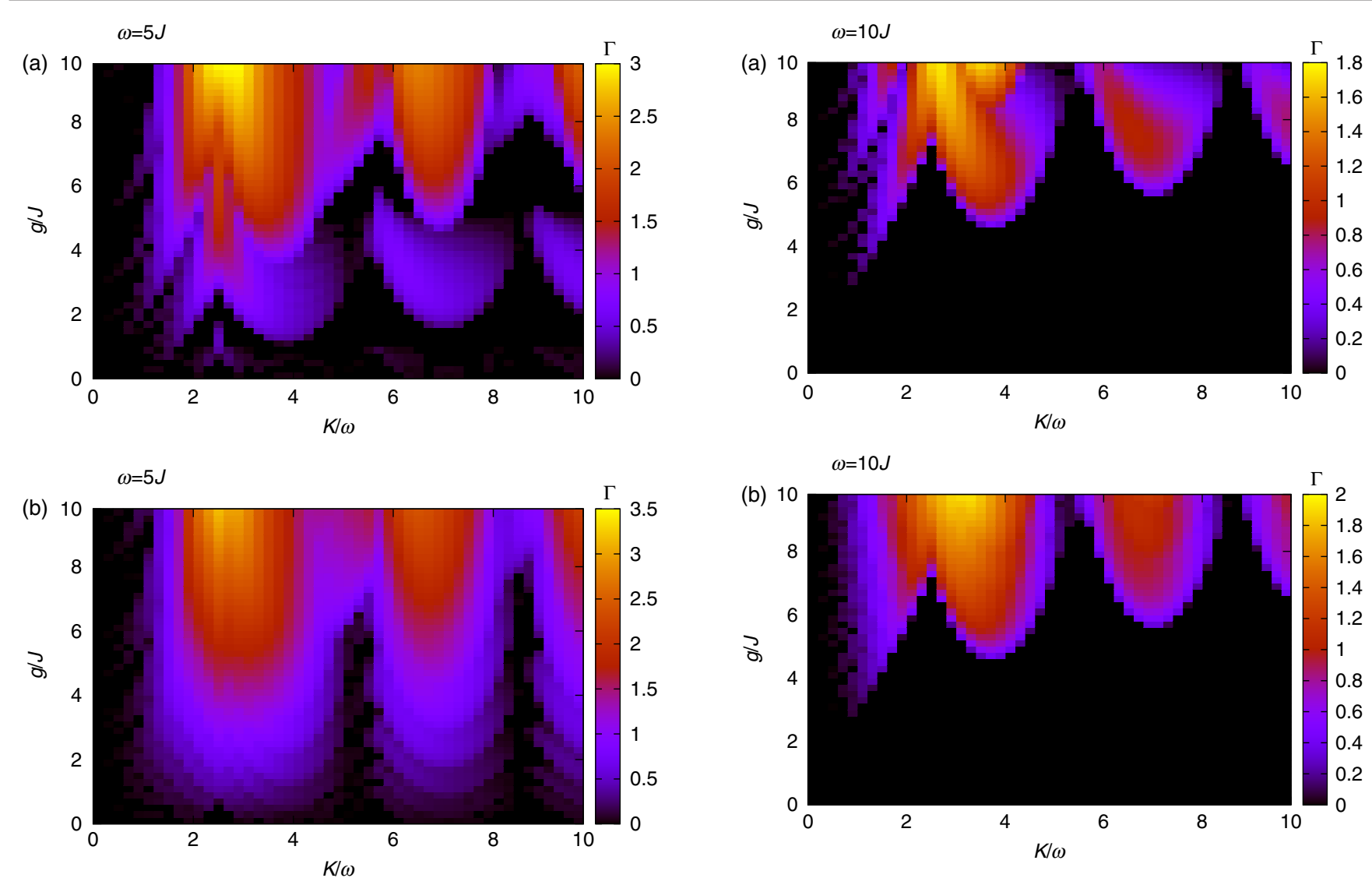

FIG. 11. Numerical instability rate as a function of interaction strength $g=U \rho$ and modulation amplitude $K / \omega$, for $\omega=5 J$ (i.e., in the low-frequency regime) and for two different numbers of lattice sites in the transverse direction: $N_{y}=4$ (top panel) and $N_{y}=16$ (bottom panel).

$$
\begin{aligned}
E_{\mathrm{av}}(\mathbf{q})= & \sqrt{\left(4\left|J_{\mathrm{eff}}\right| \sin ^{2} q_{x} / 2+4 J \sin ^{2} q_{y} / 2\right)} \\
& \times \sqrt{\left(4\left|J_{\mathrm{eff}}\right| \sin ^{2} q_{x} / 2+4 J \sin ^{2} q_{y} / 2+2 g\right)} .
\end{aligned}
$$

The resulting stability diagrams are shown in Fig. 12 for the same parameters as in Fig. 10, and they reveal an excellent agreement with the latter. Interestingly, the agreement between analytics and numerics is even better than for the 1D configuration since the presence of transverse modes in the effective dispersion Eq. (31) prevents $E_{\mathrm{av}}(\mathbf{q})$ from vanishing when $\mathcal{J}_{0}(K / \omega)=0$ [99].

\section{B. Continuous transverse degrees of freedom: The case of tubes}

We now consider the case where the transverse degree of freedom corresponds to free motion along a continuum, in one or more transverse directions. In this case, the Bogoliubov-de Gennes equations still take the form of Eq. (30), now with

FIG. 12. Analytical instability rate as a function of interaction strength $g=U \rho$ and modulation amplitude $K / \omega$, for $\omega=10 \mathrm{~J}$ (i.e., outside the low-frequency regime) and for two different numbers of lattice sites in the transverse direction: $N_{y}=4$ (top panel) and $N_{y}=16$ (bottom panel). This figure is to be compared with Fig. 10.

$$
\varepsilon(\mathbf{q}, t)=4 J \sin \frac{q_{x}}{2} \sin \left(\frac{q_{x}}{2}+p_{x}-\frac{K}{\omega} \sin \omega t\right)+\frac{\mathbf{q}_{\perp}^{2}}{2 m},
$$

so the previous analysis still holds, provided that the timeaveraged Bogoliubov dispersion $E_{\mathrm{av}}(\mathbf{q})$ is now replaced by

$$
\begin{aligned}
E_{\mathrm{av}}(\mathbf{q})= & \sqrt{\left(4\left|J_{\mathrm{eff}}\right| \sin ^{2} q_{x} / 2+\mathbf{q}_{\perp}{ }^{2} / 2 m\right)} \\
& \times \sqrt{\left(4\left|J_{\mathrm{eff}}\right| \sin ^{2} q_{x} / 2+\mathbf{q}_{\perp}{ }^{2} / 2 m+2 g\right)} .
\end{aligned}
$$

Interestingly, since this dispersion is unbounded, $\omega$ will always be smaller than the total bandwidth, and hence, there will always be (at least) one mode that is precisely set on resonance. In other words, the system necessarily falls into the low-frequency regime previously introduced, where it is unstable at any finite $g$. As explained in Sec. IV B, the instability rate can be evaluated, at lowest order, by calculating the rate on resonance associated with the mode(s) satisfying the condition $\omega=E_{\mathrm{av}}\left(\mathbf{q}^{\text {res }}\right)$ [see Eq. (26)]: 


$$
\begin{aligned}
s_{\mathbf{q}^{\text {res }}}^{*} & =4 g \mathcal{J}_{2}(K / \omega) \sin ^{2}\left(q_{x}^{\text {res }} / 2\right) J / E_{\mathrm{av}}\left(\mathbf{q}^{\text {res }}\right), \\
& =4 g \mathcal{J}_{2}(K / \omega) \sin ^{2}\left(q_{x}^{\text {res }} / 2\right) J / \omega .
\end{aligned}
$$

Importantly, although there are generically several resonant modes $\mathbf{q}^{\text {res }}$ (corresponding to different $q_{x}$ and $\mathbf{q}_{\perp}$ ), they do not have the same instability rate $s_{\mathbf{q}^{\text {res }}}^{*}$ : The total instability rate $\Gamma$ is then attributed to the most unstable mode, namely,

$$
\Gamma=\max _{\mathbf{q}^{\text {res }}}\left(s_{\mathbf{q}^{\text {res }}}^{*}\right)=s_{\mathbf{q}^{\text {mum }}}^{*},
$$

where we introduced the notation $\mathbf{q}^{\text {mum }}$ to denote the momentum of the most unstable mode.

We then identify two cases:

(i) If $\omega>\sqrt{4\left|J_{\text {eff }}\right|\left(4\left|J_{\text {eff }}\right|+2 g\right)}$, which is often the case in realistic configurations, there exists a resonant mode at $q_{x}^{\text {mum }}=\pi$, which is thus the most unstable one; $\mathbf{q}_{\perp}^{\text {mum }}$ is then adjusted so as to respect the resonance condition. The maximally unstable mode thus reads

$$
\begin{aligned}
q_{x}^{\text {mum }} & =\pi \\
\left(\mathbf{q}_{\perp}^{\text {mum }}\right)^{2} / 2 m & =\sqrt{g^{2}+\omega^{2}}-g-4\left|J_{\text {eff }}\right| .
\end{aligned}
$$

In this case, the total instability rate is given by the simple analytical formula

$$
\Gamma=4 J\left|\mathcal{J}_{2}(K / \omega)\right| \frac{g}{\omega} .
$$

(ii) If $\omega<\sqrt{4\left|J_{\text {eff }}\right|\left(4\left|J_{\text {eff }}\right|+2 g\right)}$, the most unstable mode, which obeys the resonance condition $\omega=E_{\mathrm{av}}(\mathbf{q})$, is necessarily reached at $\mathbf{q}_{\perp}^{\mathrm{mum}}=0$, yielding

$$
q_{x}^{\mathrm{mum}}=2 \arcsin \sqrt{\frac{\sqrt{g^{2}+\omega^{2}}-g}{4\left|J_{\text {eff }}\right|}} ; \quad \mathbf{q}_{\perp}^{\mathrm{mum}}=0 .
$$

In this case, the total instability rate is given by

$$
\Gamma=\left(\sqrt{g^{2}+\omega^{2}}-g\right)\left|\frac{\mathcal{J}_{2}(K / \omega)}{\mathcal{J}_{0}(K / \omega)}\right| \frac{g}{\omega} .
$$

Interestingly, these results do not depend on the number of transverse dimensions since all that matters is the existence of an unbounded continuum. Figure 13 compares the numerical stability diagram to the analytical formula Eq. (36), for realistic (experimental) parameters. As visible in Fig. 14, which shows cuts through the diagrams of Fig. 13, the agreement is excellent at low interaction or modulation amplitude, and the scaling of the instability rate is remarkably simple: $\Gamma$ increases linearly with $g$
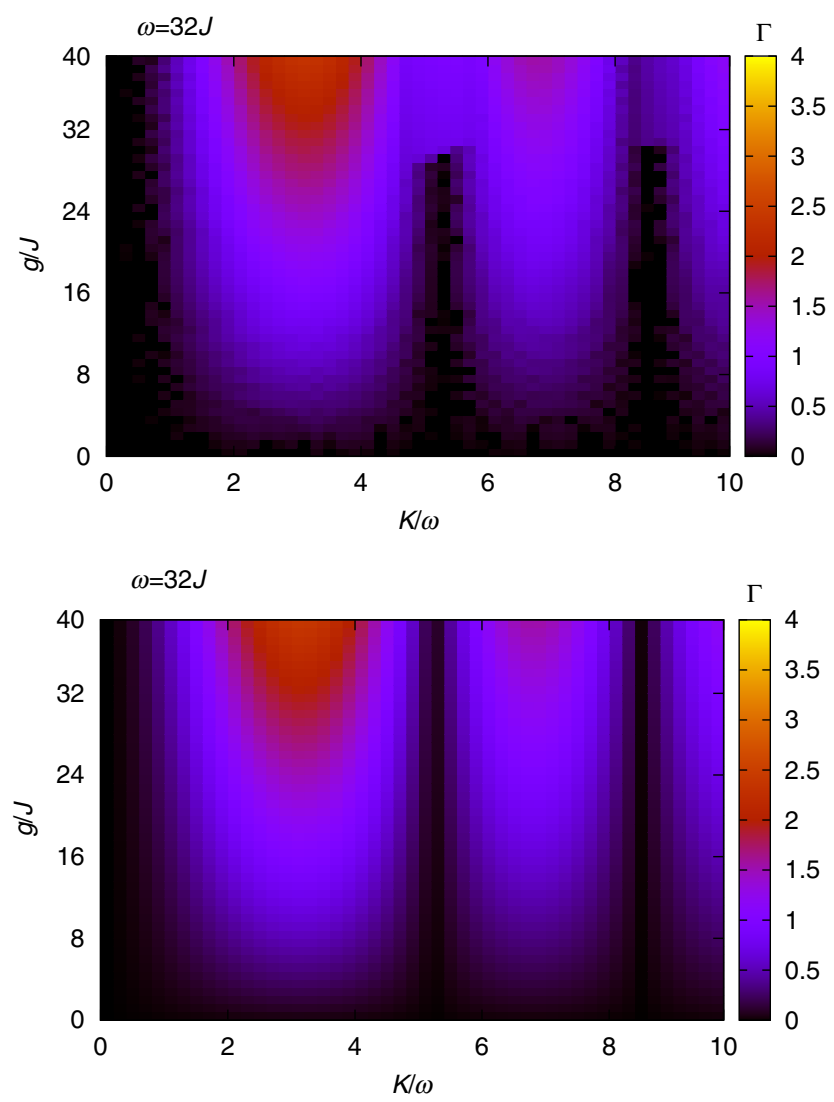

FIG. 13. Numerical (top panel) and analytical (bottom panel) instability rates $\Gamma$ as a function of the modulation amplitude $K / \omega$ and interaction strength $g$, for a high drive frequency $\omega=32 \mathrm{~J}$.

and quadratically with $K$. Such simple results could promisingly be compared with experiments. Note that in experiments, one typically has $J / \hbar \approx 100 \mathrm{~Hz}-1 \mathrm{kHz}$, so the inverse instability rates are typically of the order of $1-10 \mathrm{~ms}$.

At this stage, it is worth comparing those results with the more traditional Fermi Golden Rule (FGR) approach: First, in the present case, and contrary to what is expected for a FGR regime [63], the final rate can be inferred from the contribution of a single (well-identified) mode and not from a sum over all modes (which would then involve the density of states in the description). Second, the form of the final formula in Eq. (36) is significantly different from that resulting from a FGR argument, where the rate would typically scale as $g^{2}$ (as dictated by the squared matrix element of the perturbation [63]). When considering a driven weakly interacting degenerate Bose gas, one expects the short-time dynamics to be dominated by the parametric instability identified in this work; at longer times, as the condensate significantly depletes, heating rates are expected to be dominated by a FGR behavior [63,64], not captured in the present analysis. We believe that those two distinct mechanisms and instability regimes could be probed by current ultracold-atom experiments. 

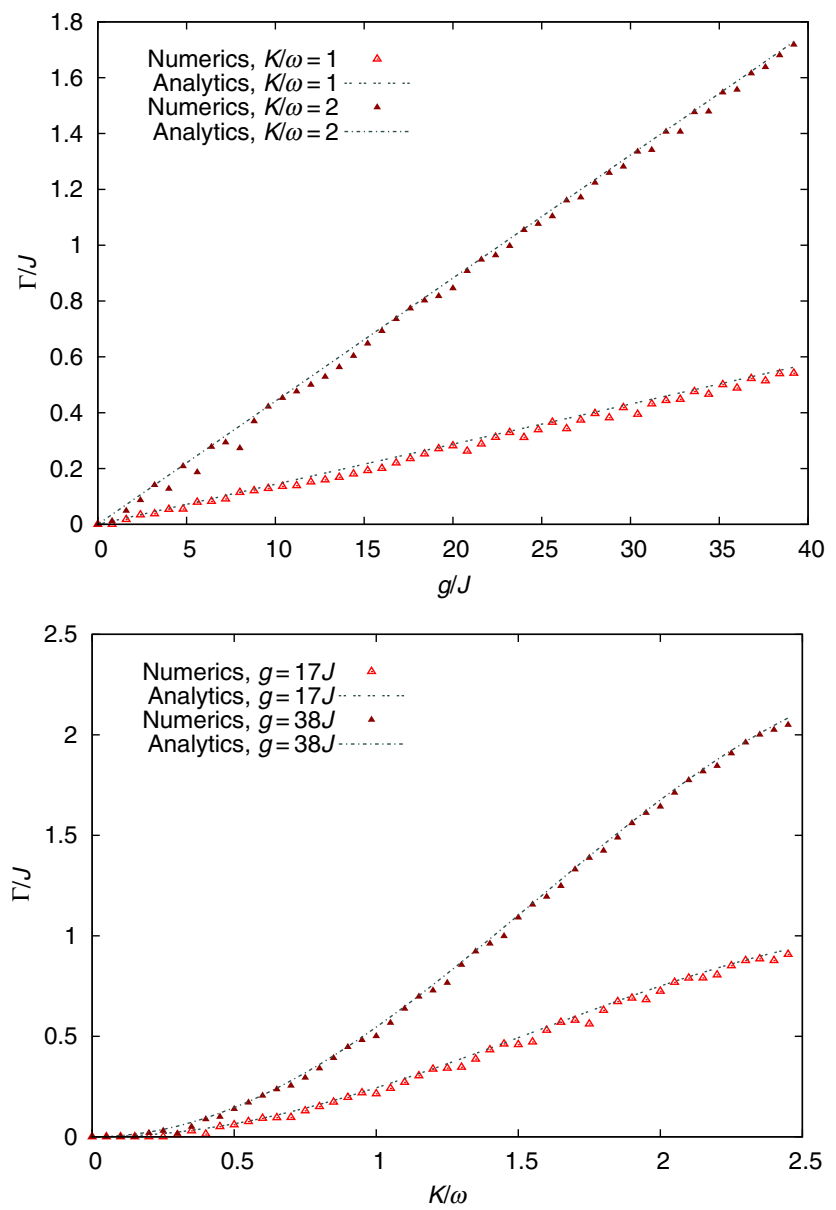

FIG. 14. Top panel: Instability rate as a function of the interaction strength $g$ for $\omega=32 J$ and for two values of the modulation amplitude. As captured by the analytical expression Eq. (36), the instability rate increases linearly with $g$. Bottom panel: Instability rate as a function of the modulation amplitude $K / \omega$ for $\omega=32 J$ and for two values of the interaction parameter $g$. As captured by the analytical expression Eq. (36), the instability rate increases quadratically with $K / \omega$ at low amplitudes.

As a practical remark, we emphasize that the results presented in this section suggest that working with a lattice seems to be more favorable than with tubes or pancakes, in view of reducing parametric instabilities in periodically driven Bose systems.

\section{PERIODICALLY DRIVEN BOSE-HUBBARD MODEL}

In this section, we discuss the relation between the Gross-Pitaevskii equation introduced in Eq. (2) and the (full quantum) driven Bose-Hubbard model [10]. Our goal is to clarify the validity of our analysis in view of describing parametric instabilities and heating in the context of this quantum model.

\section{A. Effective Hamiltonian and micromotion operator}

For the sake of concreteness, here we focus on the 1D model of Eq. (2), but the discussion applies to all models investigated in the paper (in particular, the two-dimensional models of Sec. VI). Consider a system of weakly interacting bosons, trapped in a shaken 1D lattice, as described by the periodically driven Bose-Hubbard Hamiltonian [10]

$$
\begin{aligned}
\hat{H}(t)= & -J \sum_{n}\left(\hat{a}_{n+1}^{\dagger} \hat{a}_{n}+\text { H.c. }\right)+K \cos (\omega t) \sum_{n} n \hat{a}_{n}^{\dagger} \hat{a}_{n} \\
& +\frac{U}{2} \sum_{n} \hat{a}_{n}^{\dagger} \hat{a}_{n}^{\dagger} \hat{a}_{n} \hat{a}_{n},
\end{aligned}
$$

where $J>0$ denotes the tunneling amplitude of nearestneighbor hopping, and $U>0$ is the on-site interaction strength. The on-site potential term describes a timeperiodic modulation of amplitude $K$ and frequency $\omega=2 \pi / T$.

As we already stated in the Introduction, the dynamics is stroboscopically governed by the effective Floquet Hamiltonian [see Eq. (1)]. In the absence of interactions, it is exactly [100] given by [10-12]

$$
\begin{gathered}
\hat{H}_{\text {eff }}(U=0)=-J_{\text {eff }} \sum_{n}\left(\hat{a}_{n+1}^{\dagger} \hat{a}_{n}+\text { H.c. }\right), \\
J_{\text {eff }}=J \mathcal{J}_{0}(K / \omega) .
\end{gathered}
$$

In addition, the kick operator [Eq. (1)] reads [16]

$$
\hat{K}_{\text {kick }}(t)=i \log \left[\hat{R}(t) \mathrm{e}^{-i \hat{K}_{\text {kick }}^{\text {rot }}(t)}\right] \text {, }
$$

where $\hat{R}(t)=\mathrm{e}^{-i K / \omega \sin (\omega t) \hat{X}}$ is the unitary transformation to the rotating frame (with $\hat{X} \equiv \Sigma_{n} n \hat{a}_{n}^{\dagger} \hat{a}_{n}$ the position operator on the lattice). Here, $\hat{K}_{\text {kick }}^{\text {rot }}$ denotes the kick operator in the rotating frame [16], which is explicitly given by

$$
\begin{aligned}
\hat{K}_{\text {kick }}^{\text {rot }}(t)= & -2 J \sum_{q \in \mathrm{BZ}}\left[\int_{0}^{t} \cos \left(q-K / \omega \sin \omega t^{\prime}\right) \mathrm{d} t^{\prime}\right. \\
& \left.-\mathcal{J}_{0}(K / \omega) \cos (q)\right] a_{k}^{\dagger} a_{k} .
\end{aligned}
$$

Whenever interactions are present, Eq. (40) is no longer exact, and the effective Hamiltonian is a much more complicated (possibly nonlocal) object $[59,101]$. In the high-frequency regime, it can be approximated using the inverse-frequency expansion [11], which to lowest order yields

$$
\begin{aligned}
\hat{H}_{\mathrm{eff}}= & -J_{\text {eff }} \sum_{n}\left(\hat{a}_{n+1}^{\dagger} \hat{a}_{n}+\text { H.c. }\right) \\
& +\frac{U}{2} \sum_{n} \hat{a}_{n}^{\dagger} \hat{a}_{n}^{\dagger} \hat{a}_{n} \hat{a}_{n}+\mathcal{O}\left(\omega^{-1}\right) .
\end{aligned}
$$


Hence, in the infinite-frequency limit, the periodic drive merely renormalizes the hopping matrix element $J \rightarrow J_{\text {eff }}$. Since the Bessel function takes both positive and negative values, it is possible to tune the amplitude-to-frequency ratio such that $\mathcal{J}_{0}(K / \omega)=0$, in which case tunneling is completely suppressed $[22,23,29]$. Therefore, even a weakly interacting lattice system can effectively behave as a strongly interacting one under periodic driving, in the sense that the interaction strength $U$ potentially dominates over the tunneling when $J_{\text {eff }} \approx 0$. We recall that the lattice dispersion flips sign when $\mathcal{J}_{0}(K / \omega)<0$, in which case the stable minimum appears at quasimomentum $q=\pi$ : Namely, at equilibrium, bosons are expected to condense in a finite-momentum state in this regime. We point out that corrections to the Hamiltonian (43) become nonnegligible away from the high-frequency limit and that these are expected to manifest themselves over longer time scales.

\section{B. Relation to the mean-field approach and observables}

The mean-field approach presented in this paper [based on Eq. (2)] is expected to provide a good description for the shaken 1D Bose-Hubbard model [Eq. (39)] at short times, as far as the weakly interacting regime is concerned. More specifically, if one assumes that the initial state is that of fully condensed bosons, the system in Eq. (39) can be treated within mean-field theory [102]. In this approximation, the annihilation and creation operators $\hat{a}_{n}, \hat{a}_{n}^{\dagger}$ are merely replaced by classical fields $a_{n}, a_{n}^{*}$, and the Heisenberg equations of motion for $\hat{a}_{n}$, $\hat{a}_{n}^{\dagger}$ lead to the time-dependent GPE in Eq. (2).

Throughout this work, our choice for the initial state corresponds to the Bogoliubov ground state of the effective Hamiltonian (43) [see Sec. II]. In order to compute heating rates for such a model, following the procedure detailed in Sec. III B, we also had to fix the initial condition for the fluctuation term $\delta a$, which, in our GPE-based approach, could be taken to be an arbitrary small perturbation on top of the condensate.

However, our choice for the initial condensate wave function (i.e., ground state of $\hat{H}_{\text {eff }}$ ) suggests a natural choice for the initial fluctuation term $\delta a(t=0)$. Indeed, one could assume that at $t=0$, this fluctuation term is precisely given by the Bogoliubov wave function of a condensate in the ground state of $\hat{H}_{\text {eff }}$, as obtained from a numerical diagonalization of the corresponding (effective) Bogoliubov equations, namely,

$$
\delta a_{n}(t=0)=\sum_{q} u_{q} \mathrm{e}^{i q n}+v_{q}^{*} \mathrm{e}^{-i q n}
$$

where $u_{q}, v_{q}$ correspond to the solution of

$$
\left(\begin{array}{cc}
\varepsilon_{\mathrm{av}}(q)+g & g \\
-g & -\varepsilon_{\mathrm{av}}(-q)-g
\end{array}\right)\left(\begin{array}{c}
u_{q} \\
v_{q}
\end{array}\right)=E_{\mathrm{av}}(q)\left(\begin{array}{c}
u_{q} \\
v_{q}
\end{array}\right),
$$

where $\varepsilon_{\text {av }}(q)$ and $E_{\text {av }}(q)$ depend on the model under consideration. Here, $\varepsilon_{\mathrm{av}}(q)$ denotes the time average of the instantaneous dispersion $\varepsilon(q, t)$ [see Eq. (10) for the 1D model, and Eq. (30) for the 2D case], and $E_{\mathrm{av}}(q)$ is the time-averaged Bogoliubov dispersion [see Eq. (12) for the 1D model, and Eq. (31) for the 2D case].

A relevant observable to quantify heating and losses, which can be probed in experiments, is the noncondensed density, which, in the Bogoliubov approximation, reads [103]

$$
\rho_{n c}(t)=\frac{1}{\operatorname{Vol}} \sum_{q}\left|v_{q}(t)\right|^{2}
$$

with Vol the volume of the system. Given the above initial condition, it is straightforward to apply the numerical tools developed in Sec. III B [104], so as to describe the time evolution of the noncondensed density Eq. (45) in the system. For instance, Fig. 15 shows the exponential growth rate of the noncondensed fraction (referred to as the loss rate) obtained for the 2D lattice model of Sec. VI A, which is therefore expected to describe particle losses in the associated 2D-driven Bose-Hubbard model. Note that, unsurprisingly, this is very similar to the stability diagram, Fig. 11(b), which corresponds to the same parameters (see Sec. III B).

The aforementioned aspects are intrinsic to the meanfield Bogoliubov approximation in the frame of the model under consideration. More generally, there are a few important points one has to keep in mind, when adopting a mean-field approach to study out-of-equilibrium

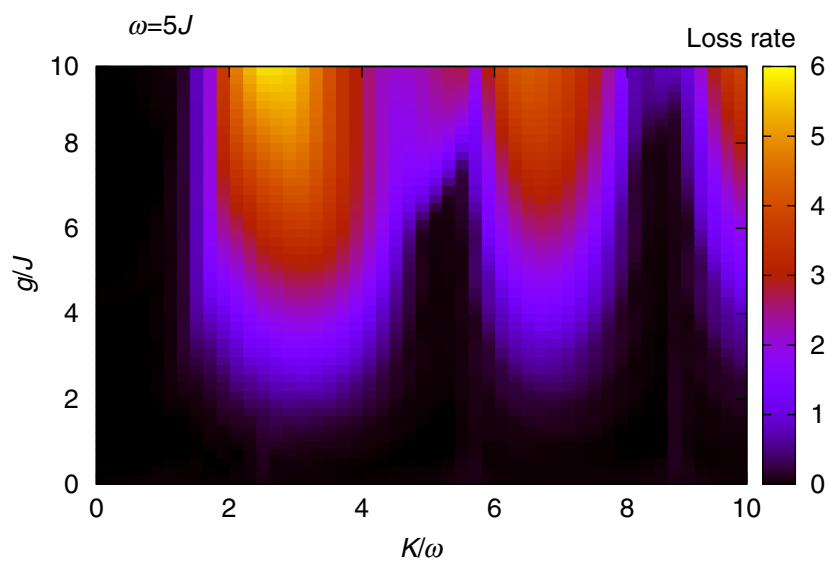

FIG. 15. Loss rate (exponential growth rate of the noncondensed density) for the 2D lattice model of Sec. VI A, for $\omega=5 \mathrm{~J}$ and $N_{y}=16$. This rate is obtained by fitting the exponential growth of the noncondensed fraction, similarly to what was done in Fig. 5 for the energy. 
ergodic (nonintegrable) bosonic systems subject to periodic driving:

(i) As already alluded to above, the Floquet Hamiltonian becomes an increasingly nonlocal operator, the further the drive frequency deviates from the infinite-frequency limit. An intriguing and interesting part of this intrinsic nonlocality is due to Floquet many-body resonances [105] appearing because of the hybridization of many-body states induced by the drive. These resonances appear as a result of energy absorption and provide shortcuts between states in energy space separated by multiples of the drive frequency. Hence, in the unstable phase, they are expected to affect the heating rates at times $t \sim U^{-1}$, when interaction effects between quasiparticles become important.

(ii) In fact, one can anticipate (using the inversefrequency expansion in the rotating frame) that the Floquet Hamiltonian of the driven BoseHubbard model contains complicated three- and higher-body interaction terms, starting from order $\omega^{-3}$. It is currently unknown how these terms modify and limit the application of Bogoliubov's mean-field theory associated with the effective (Floquet) Hamiltonian.

Despite the open character of these potential issues related to interacting bosonic systems, we expect that our GPEbased analysis captures the dominant contribution to the short-time evolution of the periodically driven BoseHubbard model in Eq. (39), in particular, the onset of instability.

\section{Time evolution beyond the linearized regime: The conserving approximation}

Since the mean-field Bogoliubov approximation assumes a macroscopic occupation of the condensate mode, it remains valid provided the number of noncondensed atoms remains small compared to the total number of atoms throughout the entire subsequent evolution. As expected, this assumption fails in the unstable regions, where the depletion of the condensate grows exponentially and the mean-field approach typically holds at short times. Thus, the time scale for reaching a sizable dynamical depletion sets a natural upper bound on the validity of mean-field approaches.

One way to avoid the problems associated with particle conservation is to apply the weak-coupling conserving approximation (WCCA) [74]. Based on a Keldysh field theory formalism, the WCCA is the minimal extension of Bogoliubov theory, which includes the proper effective interactions between the Bogoliubov quasiparticles and the condensate, order by order in the original interaction strength $U$, while ensuring particle conservation at all times. Truncated to linear order in $U$, the WCCA is equivalent to the Bogoliubov-Hartree-Fock approximation [106], and in the following, we restrict ourselves to this case. The unknown variables in the WCCA formalism are [107]

$$
\begin{aligned}
\phi(t) & =e^{-i \int_{0}^{t} \mu\left(t^{\prime}\right) \mathrm{d} t^{\prime}}\left\langle a_{q=0}\right\rangle, \\
F_{11}(t ; q) & =\frac{1}{2}\left\langle\left\{a_{q}(t), a_{q}^{\dagger}(t)\right\}\right\rangle_{c}, \\
F_{12}(t ; q) & =\frac{e^{-2 i \int_{0}^{t} \mu\left(t^{\prime}\right) \mathrm{d} t^{\prime}}}{2}\left\langle\left\{a_{q}(t), a_{-q}(t)\right\}\right\rangle_{c},
\end{aligned}
$$

where $\phi(t)=a_{n}^{0}(t) e^{-i \int_{0}^{t} \mu\left(t^{\prime}\right) \mathrm{d} t^{\prime}} \sqrt{\mathrm{Vol}}$ is the $q=0$ mode of the spatially homogeneous (i.e., $n$-independent) condensate wave function, and where $\mu(t)=-z J \cos (K / \omega \sin \omega t)+g$ is the chemical potential, which is irrelevant for U(1)conserving dynamics. The subscript $c$ stands for connected correlators: $\langle A(t) B(t)\rangle_{c}=\langle A(t) B(t)\rangle-\langle A(t)\rangle\langle B(t)\rangle$. The equal-time correlator $F_{11}(t ; q)$ is, apart from an additive constant, the phonon density. In momentum space, it is related to the momentum distribution function of the quasiparticles $n_{q}(t)$ by $n_{q}(t)=F_{11}(t ; q)-1 / 2$. Note that $n_{q}(t)$ does not include the condensate delta function peak at $q=0$. The WCCA EOM for the equal-time correlators represents a simple system of nonlinear and nonlocal in space equations

$$
\begin{aligned}
i \partial_{t} \phi(t)= & \varepsilon_{q=0}(t) \phi(t)+\frac{U}{\mathrm{Vol}}\left[[\phi(t)]^{*}[\phi(t)]^{2}+2 \phi(t) \int_{q^{\prime}} F_{11}\left(t ; q^{\prime}\right)+[\phi(t)]^{*} \int_{q^{\prime}} F_{12}\left(t ; q^{\prime}\right)\right], \\
\partial_{t} F_{11}(t ; q)= & 2 \operatorname{Im}\left\{\frac{U}{\operatorname{Vol}}\left([\phi(t)]^{2}+\int_{q^{\prime}} F_{12}\left(t ; q^{\prime}\right)\right)\left[F_{12}(t ; q)\right]^{*}\right\}, \\
i \partial_{t} F_{12}(t ; q)= & \left\{\left[\varepsilon_{q}(t)+\varepsilon_{-q}(t)\right] F_{12}(t ; q)+2 \frac{U}{\operatorname{Vol}}\left[2\left(|\phi(t)|^{2}+\int_{q^{\prime}} F_{11}\left(t ; q^{\prime}\right)\right) F_{12}(t ; q)\right.\right. \\
& \left.\left.+\left([\phi(t)]^{2}+\int_{q^{\prime}} F_{12}\left(t ; q^{\prime}\right)\right) F_{11}(t ; q)\right]\right\},
\end{aligned}
$$

where ${ }^{*}$ denotes complex conjugation. 
The conserved quantity itself is the total number of particles (condensed and excited):

$$
\begin{aligned}
N & =|\phi(t)|^{2}+\int_{q} n_{q}(t) \\
& =|\phi(t)|^{2}+\int_{q}\left(F_{11}(t ; q)-\frac{1}{2}\right)=\text { const. }
\end{aligned}
$$

One recognizes the GPE equation for the spatially homogeneous condensate density $\phi(t)$ and identifies the additional phonon feedback terms. Quite generally, if one eliminates all terms containing integrals over $q$, the WCCA EOM decouples into the familiar GPE for the condensate [see Eq. (2)], while the second two equations are equivalent to the BdG equations, Eq. (44) [108].

Opening up a channel between the quasiparticles and the condensate, we find that the phonon buildup rate decreases (and, consequently, the condensate depletion slows down) compared to the linearized $\mathrm{BdG}$ equations, in agreement with intuitive expectations. In other words, the WCCA deviates from the exponentially growing linearized solution, which sets a natural scale on the validity of the meanfield approximation.

To justify the applicability of the mean-field approach at short times, we compare the dynamical buildup of quasiparticle excitations in the parametrically unstable regime, predicted by the linearized EOM [see Eq. (9)], and the WCCA (to order $U$ ). Here, we initialize the system in the Bogoliubov ground state corresponding to the nondriven Hamiltonian $H(K=0)$. Figure 16 shows the time evolution of the quasiparticle excitations (top panel) and the condensate density (bottom panel) for the 2D model of Sec. VI B. One can clearly identify two stages of evolution: In stage I, which continues for about $t \lesssim 220 T$ driving periods, the dynamics is governed by the single-particle mean-field physics. The dotted vertical line at $t^{*} \approx 60 T$ marks the time up to which the dynamics predicted by the linearized mean-field equations agrees with the WCCA. Thus, for $t \leq t^{*}$, this initial regime features an exponential buildup of phonons at the momenta satisfying the resonant condition $\omega=E_{\mathrm{av}}(\mathbf{q})$. For $60 T \lesssim t \lesssim 220 T$, the time evolution is characterized by a different growth rate than the one predicted by the mean-field equations for this system configuration. By the time $t \approx t^{*}$, the backaction effect of the quasiparticles onto the condensate becomes sizable, and the dynamics no longer follows the initial linearized exponential growth. Based on the available data, it is not possible to determine whether the growth remains exponential or follows another law. In stage II, $t \gtrsim 220 T$, the population of the resonant $q$ modes slows down significantly, although it never really saturates, as can be seen from the condensate depletion at longer times [see Fig. 16 (bottom panel)]. Because of the unbounded character of the dispersion along the $q_{y}$ direction, the presence of resonant modes set by the condition $\omega=E_{\mathrm{av}}(\mathbf{q})$ does not allow for the formation of a Floquet steady state. Nevertheless, the
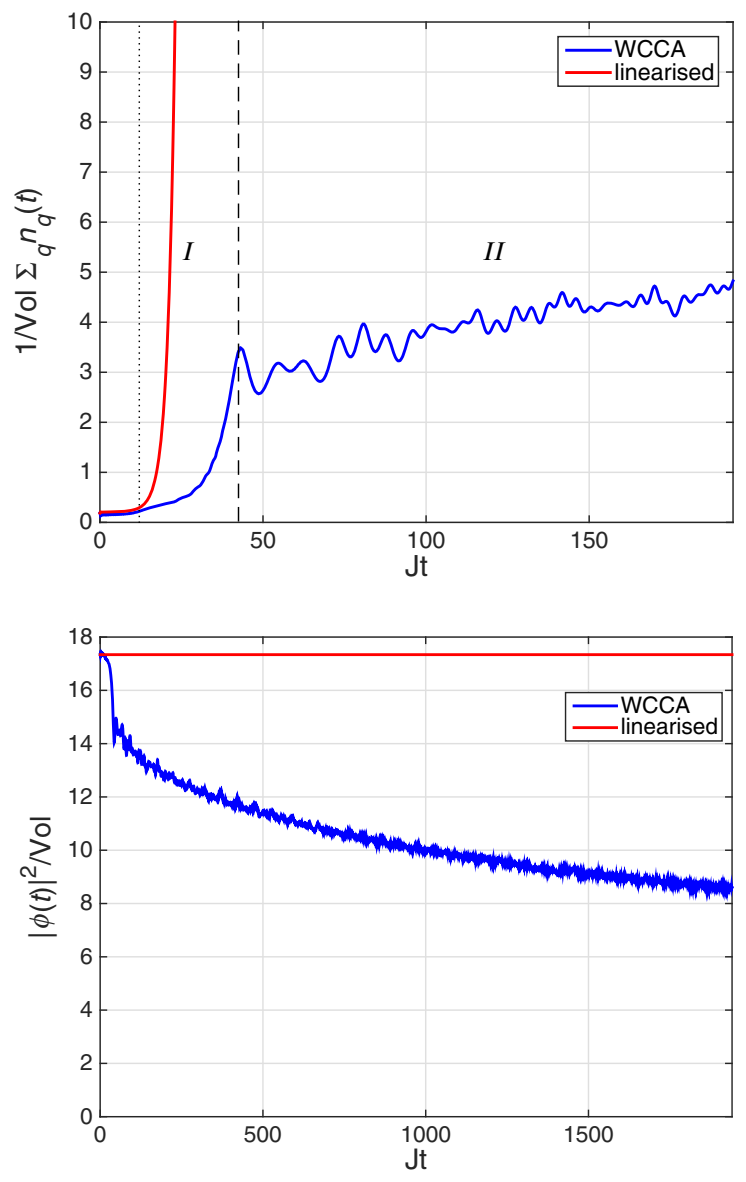

FIG. 16. Time dependence of the quasiparticle (phonon) density (top panel) and the condensate density (bottom panel) using the linearized (red line) and the conserving (blue line) approximation, for the $2 \mathrm{D}$ band model from Sec. VI B (with a lattice along the $x$ direction and the continuum along the $y$ direction). There are two discernible regimes: I and II (see text), delineated by a vertical dashed line. The vertical dotted line marks the time up to which the linearized mean-field dynamics and the WCCA agree well. The model parameters are $\rho / \mathrm{Vol}=17.34$, $U / J=1.011(g / J=17.53), m=1 /(20 J)$, and healing length to $x$-lattice constant ratio $\xi / a_{x}=1.47, \omega / J=32.37$, and $K / \omega=1.0$. We use a total of $N=N_{x} N_{y}=5 \times 10^{4}$ momentum points with $N_{x}=50$ and $N_{y}=1000$ to reach the thermodynamic limit. As an initial state, we choose the Bogoliubov ground state.

growth rate diminishes significantly, and the dynamics slows down, as expected for a prethermal regime. Interestingly, the condensate evolution curve [Fig. 16 (bottom panel)] changes curvature in stage II, compared to stage I. Curiously, we note that a very similar change of behavior was reported in a cosmological context [77].

Important features of the quasiparticle dynamics are conveniently reflected in their momentum distribution function $n_{q}(t)$ [109]. Figure 17 shows a comparison between the momentum distributions, as obtained by time evolving the linearized and WCCA EOM over a long duration $(t=1000 T)$, for the same 2D model. The visible lines, which materialize largely populated modes, 


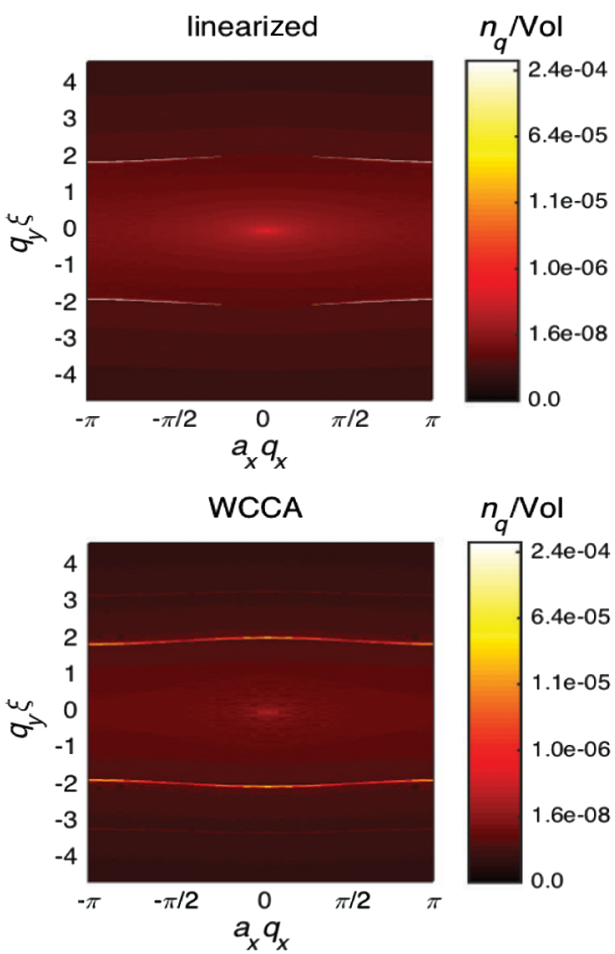

FIG. 17. Quasiparticle momentum distribution function $n_{q}$ after a duration of $t=1000 T$, for the 2D band model from Sec. VI B (with a lattice along the $x$ direction and continuum along the $y$ direction). The top panel shows the linearized mean-field model, while the bottom panel shows the conserving approximation (WCCA) to order $U$. The model parameters are the same as in Fig. 16. Here, we set the lattice constant $a_{x}=1$ to unity, while $\xi=1.47 a_{x}$ denotes the BEC healing length.

correspond to on-resonance modes $\left[E_{\mathrm{av}}(\boldsymbol{q})=\omega\right]$, which have indeed been shown to be unstable. In the linearized dynamics, the largest peaks are visible around the maximally unstable modes, as predicted by our theory [see Eqs. (35) and (37)]; for the considered parameters, the most unstable mode indeed corresponds to $q_{x}^{\mathrm{mum}}= \pm \pi$, and $q_{y}^{\text {mum }}$ is adjusted so that the resonance condition $\omega=$ $E_{\mathrm{av}}(\mathbf{q})$ is fulfilled (see Sec. VIB). We stress that the large width of the peaks around $q_{x}= \pm \pi$, in the top panel of Fig. 17, is an artifact due to the significant population of momentum modes at long times, which yields saturation on this plot; we refer to Fig. 1 for a more striking illustration of these peaks, as calculated at shorter times (for the same model parameters). In contrast, the WCCA couples all momenta through the $q^{\prime}$ integrals in Eq. (47), allowing the population to further spread along the $x$ axis. All modes fulfilling the resonance condition now exhibit peaks of similar magnitudes, precisely materializing the curve of equation $\omega=E_{\mathrm{av}}(\mathbf{q})$; note, though, that the populations of the modes along this curve are not completely homogeneous, presumably due to the nonlinear character of the WCCA EOM and/or the differences in the instability rates of resonant modes. Moreover, as we discussed in Sec. IV C, modes that are not precisely on resonance, but sufficiently close to it, are also affected by the instability.

A summary of the various behavior types of the momentum distribution, which are expected for the different parameter regimes, is presented in Sec. VIII (see Figs. 19 and Table II).

We conclude this section by noticing that while the WCCA to leading order in $U$ features a prethermal Floquet regime at high driving frequencies [74], the absence of quasiparticle collisions prevents the onset of thermalizing dynamics at later times. Including the next-to-leading order in $U$ has been done for the periodically driven $O(N)$ model $[110,111]$, where the crossover from a prethermal steady state to a thermal regime becomes clearly visible.

\section{SUMMARY OF THE MAIN RESULTS AND CONCLUDING REMARKS}

The analysis presented in this work reveals the crucial role played by parametric instabilities in the short-time dynamics of periodically driven band models. It provides a quantitative description and a qualitative understanding of the stability diagrams of these periodically driven systems, capturing both the stability boundaries and the instability rates in its vicinity. This work also identifies clear signatures of these early-stage instabilities, which could be detected in current cold-atom experiments.

On the one hand, the quantitative description of the instability rates will prove helpful to guide experimentalists in finding stable regimes of operation. A first indicator is the instability rates themselves. Table I summarizes the scaling behavior found for instability rates in the thermodynamic limit as a function of the model parameters, as derived in Sec. IV C. Remarkably, these scaling laws hold in all cases investigated in this work, i.e., 1D and 2D lattices and high- and low-frequency regimes.

Other important information concerns the identification of the most unstable mode $\mathbf{q}^{\text {mum }}$. Table II recalls the characteristics of this most unstable mode for the various

TABLE I. Limiting scalings of instability rates in the thermodynamic limit, in terms of the interaction strength $g$, the modulation amplitude $K$, and frequency $\omega$. The high- and low-frequency regimes are defined through a comparison between $\omega$ and the free-particle effective bandwidth (see Sec. III A).

\begin{tabular}{|c|c|c|c|}
\hline \multicolumn{2}{|c|}{ Low $g$} & Low $K$ & Low $\omega$ \\
\hline High-frequency regime & Low-frequency regime & & \\
\hline$\Gamma \propto \sqrt{\left(g-g_{c}\right)}$ & $\Gamma \propto g$ & $\Gamma \propto K^{2}$ & $\Gamma \propto \omega \times f(K / \omega)$ \\
\hline
\end{tabular}


TABLE II. Characterization of the most unstable mode (mum) for various relevant regimes. The low-frequency regime is generically defined through the criterion according to which the drive frequency $\omega$ is smaller than the effective free-particle bandwidth (e.g., $\omega<4\left|J_{\text {eff }}\right|$ for the $1 \mathrm{D}$ shaken-lattice model of Sec. III A). We stress that the effective free-particle dispersion is unbounded for models involving a continuous spatial dimension, in which case the system necessarily corresponds to the low-frequency regime (for all $\omega$ ).

\begin{tabular}{|c|c|c|}
\hline \multicolumn{2}{|c|}{ Low-frequency regime } & \multirow[t]{2}{*}{ High-frequency regime } \\
\hline$\omega<\sqrt{4\left|J_{\text {eff }}\right|\left(4\left|J_{\text {eff }}\right|+2 g\right)}$ & $\omega>\sqrt{4\left|J_{\text {eff }}\right|\left(4\left|J_{\text {eff }}\right|+2 g\right)}$ & \\
\hline $\begin{array}{l}q_{x}^{\operatorname{mum}}<\pi \\
q_{\perp}^{\text {mum }}=0 \\
\text { See Eq. (37) }\end{array}$ & $\begin{array}{c}q_{x}^{\text {mum }}=\pi \\
q_{\perp}^{\text {mum }}>0 \\
\text { See Eq. }(35)\end{array}$ & $\begin{array}{c}q_{x, y, z}^{\operatorname{mum}}=\pi \\
\text { (at the onset of instability) }\end{array}$ \\
\hline
\end{tabular}

relevant regimes discussed in this work. In the highfrequency regime, where $\omega$ is larger than the free-particle effective bandwidth, the onset of instability is necessarily driven by the mode $q=\pi$ (or $q_{x, y, z}=\pi$ if several lattice degrees of freedom are present). In the low-frequency regime, the most unstable mode $\mathbf{q}^{\text {mum }}$ obeys the resonance condition $\omega=E_{\mathrm{av}}(\mathbf{q})$. Yet, in dimensions higher than one, we found that this condition is not sufficient to unambiguously determine $\mathbf{q}^{\text {mum }}$ since a whole set of resonant modes (associated with different rates) typically satisfy it. Our analysis showed that two situations can occur and that these could be distinguished by comparing the frequency $\omega$ to the effective Bogoliubov bandwidth associated with the shaken-lattice direction alone: If $\omega>$ $\sqrt{4\left|J_{\text {eff }}\right|\left(4\left|J_{\text {eff }}\right|+2 g\right)}$, then $q_{x}^{\text {mum }}=\pi$ and $q_{\perp}^{\text {mum }} \neq 0$ [see Eq. (35)]; conversely, if $\omega<\sqrt{4\left|J_{\text {eff }}\right|\left(4\left|J_{\text {eff }}\right|+2 g\right)}$, we find $q_{x}^{\mathrm{mum}}<\pi$ and $q_{\perp}^{\mathrm{mum}}=0$ [see Eq. (37)]. Let us emphasize that, in principle, the so-called low-frequency regime can be reached at arbitrarily large frequencies; for instance, this typically occurs whenever the free-particle effective dispersion is unbounded, as in the case where a continuous direction is present. Figures 18 and 19 show the momentum distribution as calculated for the hybrid-2D band model of Sec. VI B (i.e., a shaken lattice along the $x$ direction and a continuum along the $y$ direction); by exploring the different parameter regimes, these results illustrate all the behaviors described above (Table II).

Those predictions on the instability rates can readily be tested in present-day experiments. For typical experimental parameters, the ratio $\omega / J$ is generally set in the range 10-100; therefore, in the case of pure-lattice systems, experiments would typically lie in the high-frequency regime defined in Sec. III A, and the most unstable mode is thus expected to be $q_{x, y, z}^{\mathrm{mum}}=\pi$. In turn, experiments with a continuous degree of freedom fall into the second column of Table II, and we thus expect the maximal instability to occur through $q_{x}^{\text {mum }}=\pi$ and finite $q_{\perp}^{\text {mum }}$. We point out that experiments could also investigate the low-frequency regime of driven-lattice systems (first column of Table II), by working with lower drive frequencies $\omega \sim J$ (see, e.g., Ref. [28]).

The different observables that we introduced are accessible in current experiments, and probing them should allow clear experimental signatures of the predicted instability behavior. In particular, measuring the momentum distribution of the gas, which is routinely performed through time-of-flight techniques, and identifying the positions of the peaks would give us access to the momentum of the most unstable modes, which is one of the most recognizable characteristics of parametric instabilities.

On the other hand, several important conceptual points arise from the detailed analysis presented in this paper: In particular, the intuitive stability criterion $\omega>2 W_{\text {eff }}$ is not sufficient to properly estimate the instability boundary and rates in the system, as becomes clear from the rigorous derivation we provided. More precisely, the parametric resonance condition for the instability is fulfilled for $\omega=E_{\mathrm{av}}(q)$, but in addition, one has to take the (possibly) large width of the resonance window into account in order
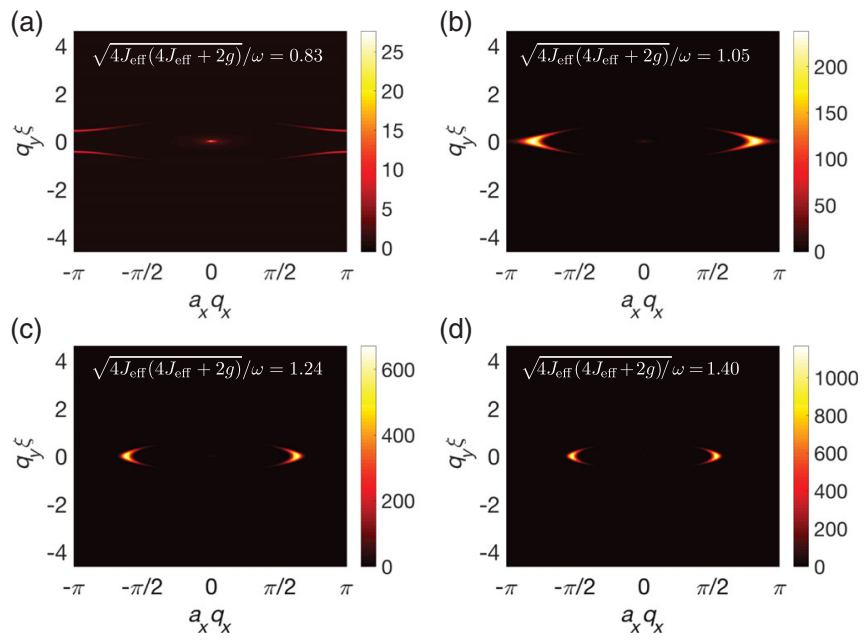

FIG. 18. Quasiparticle momentum distribution function $n_{q}$ after a duration of $t=10 T$, for the 2D band model from Sec. VI B (with a lattice along the $x$ direction and a continuum along the $y$ direction), computed using the linearized dynamics, for different values of the relevant parameter $\sqrt{4\left|J_{\text {eff }}\right|\left(4\left|J_{\text {eff }}\right|+2 g\right)} / \omega$; see Table II. The suggested ellipses correspond to on-resonance modes $\left[E_{\mathrm{av}}(q)=\pi\right]$, which are unstable, while the most unstable modes, which are revealed by the main peaks in the momentum distribution, are accurately described by our theoretical predictions (see Table II). 

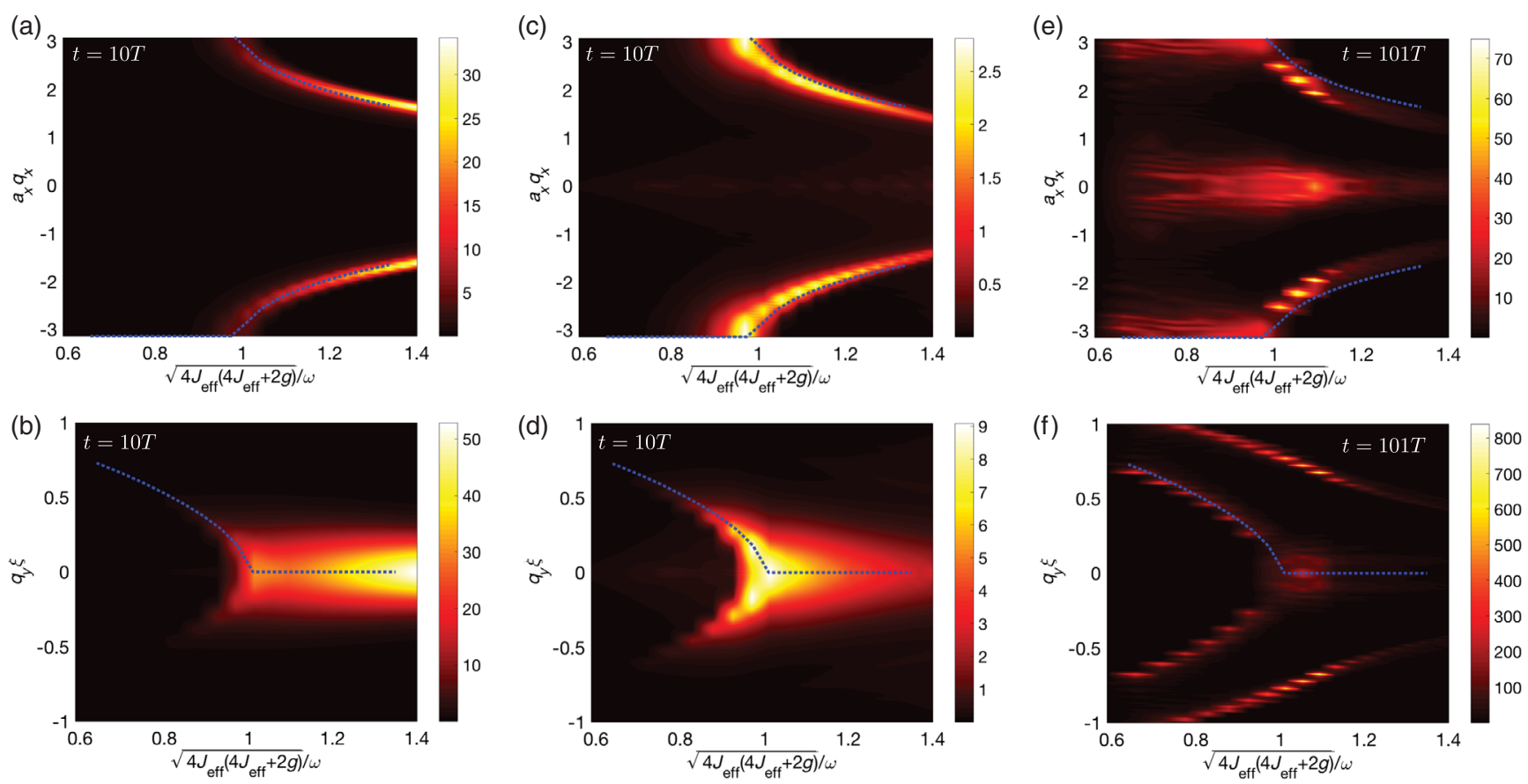

FIG. 19. Quasiparticle momentum distribution function $n_{q}$ for the 2D band model from Sec. VI B (with a lattice along the $x$ direction and a continuum along the $y$ direction), as a function of the parameter $\sqrt{4\left|J_{\text {eff }}\right|\left(4\left|J_{\text {eff }}\right|+2 g\right)} / \omega$ and of $q_{x}$ (upper line) [resp. $q_{\perp}$ (lower line)], after integration over $q_{\perp}$ (resp. $q_{x}$ ), and computed as follows: (left panels) after a time $t=10 T$ using the linearized dynamics, (middle panels) same time but using the WCCA, and (right panels) after a longer time $t=101 T$ using the WCCA. The dotted blue line indicates our theoretical prediction for the most unstable mode (see Table II), which is verified in all regimes: For $\sqrt{4\left|J_{\text {eff }}\right|\left(4\left|J_{\text {eff }}\right|+2 g\right)} / \omega>1$, we find $q_{\perp}^{\text {mum }}=0$ and $q_{x}^{\text {mum }}<\pi$, as predicted by Eq. (37); for $\sqrt{4\left|J_{\text {eff }}\right|\left(4\left|J_{\text {eff }}\right|+2 g\right)} / \omega<1$, we find $q_{x}^{\text {mum }}=\pi$ and $q_{\perp}^{\text {mum }}>0$, as given by Eq. (35). Note that, in the latter regime, the peaks are only apparent after a long time (here $101 T$, right panel) because of the small values of the corresponding instability rates; see also Fig. 18(a), where these peaks are made visible. As already observed in Fig. 16, both linearized and WCCA agree very well on the position of the peaks, although the WCCA predicts a broadening of the instability zone due to nonlinear couplings between the modes. Note that at longer times (right panel), higher-order parametric resonances are also visible. The apparent "quantized" character of the resonant peaks for $t=101 T$ is a numerical artifact due to the discretization of the variable $\sqrt{4\left|J_{\text {eff }}\right|\left(4\left|J_{\text {eff }}\right|+2 g\right)} / \omega$ used in the numerical simulation.

to properly estimate the position of the boundary; we note that similar effects were found in the context of the manybody Kapitza pendulum [75].

As a result of our study, the following general guidelines deserve to be highlighted: In lattice systems, working at high frequency (much larger than the effective bandwidth of Bogoliubov excitations) is favorable since no parametric resonance can then occur, ensuring a stable evolution. We also analyzed the effect of transverse directions, be it a deep lattice or tubes; although the instability is generically increased in both cases by the fact that more and more modes are available in the system (offering more possibilities to have resonances), we showed that working with a lattice should be much more favorable to find stable regimes and have less heating. We stress that our analysis results from a tight-binding approximation, namely, the restriction of the study to a single Bloch band. This simplification is reasonable for situations where the drive frequency is set away from any interband resonances, which is indeed possible in experiment. Interestingly, we note that such interband excitations have been generated and studied experimentally in Ref. [112]. The interplay between interband transitions, as generated by setting the drive frequency close to resonance with interband spacing, and the existence of parametric instabilities should show rich behaviors, which could be explored through the theoretical approach presented in this work.

Finally, we emphasize that the instability rates derived from parametric resonances (see, e.g., the scalings in Table I) differ quantitatively from the ones computed within a FGR approach [64], which generically scale as $g^{2}$ for low interactions. In particular, we note that contrary to the case of parametric resonances, the rates emanating from the FGR approach strongly depend on the dimensionality of the system, through its density of states, which indicates that the two phenomena are indeed very distinct in essence. Since the system is bosonic, we expect that the parametric instability should dominate the short-time dynamics because of its exponential growth rate. It is only at later times that Fermi's golden rule comes to rule the behavior of the system, when the dynamics will be dominated by collision processes leading to thermalization 
at a higher temperature, a feature that is generically known for quantum Bose fluids [77]. Hence, both phenomena take place in different regimes of the time evolution. More precisely, for typical experimental parameters, the instability rates derived from parametric resonances are found to be of the order of $1-10 \mathrm{~ms}$, while the Fermi golden rule decay usually spreads over times of the order of $100 \mathrm{~ms}$ [64]. It is within the scope of present-day experiments to observe those two distinct regimes.

\section{ACKNOWLEDGMENTS}

The authors are pleased to acknowledge J. Berges, I. Bloch, J. C. Budich, J. Dalibard, M. Dalmonte, P. Gaspard, M. Lacki, C. Laflamme, J. Lutsko, J. Nager, S. Nascimbene, H. Pichler, A. Polkovnikov, M. Reitter, U. Schneider, K. Wintersperger, and P. Zoller for insightful discussions. The work in Brussels is supported by the ERC Starting Grant TopoCold, the FRS-FNRS (Belgium), the Université Libre de Bruxelles (ULB) and the Belgian Science Policy Office (PAI Project No. P7/18). M. B. acknowledges support by NSF Grant No. DMR-1506340 and Grant No. ARO W911NF1410540. E. D. was supported by Harvard-MIT CUA, NSF Grant No. DMR1308435, AFOSR Quantum Simulation MURI, and AFOSR MURI Photonic Quantum Matter. Part of the computational work reported in this paper was performed on the Shared Computing Cluster, which is administered by Boston University's Research Computing Services. The authors also acknowledge the Research Computing Services group for providing consulting support, which has contributed to the results reported within this paper.

\section{APPENDIX A: DIFFERENT PARAMETRIZATIONS OF THE BOGOLIUBOV EXPANSION IN THE TIME-DEPENDENT CASE}

In this appendix, we recall the different parametrizations that are commonly used for the Bogoliubov expansion in time-dependent systems. Starting from the time-dependent GPE on the lattice,

$$
i \partial_{t} a_{n}=-J\left(a_{n+1}+a_{n-1}\right)+K \cos (\omega t) n a_{n}+U\left|a_{n}\right|^{2} a_{n},
$$

and given the time-dependent solution for the condensate wave function $a_{n}^{(0)}(t)$, the basic idea of the Bogoliubov expansion is to consider a small perturbation on top of $a_{n}^{(0)}(t)$.

A natural approach consists in writing $a_{n}(t)=a_{n}^{(0)}(t)+$ $\delta a_{n}(t)$, and to linearize the Gross-Pitaevskii equation in terms of $\delta a_{n}$. This yields the time-dependent BdG equations, which take the general form

$$
i\left(\begin{array}{c}
\dot{\delta} a_{n} \\
\dot{\delta a_{n}^{*}}
\end{array}\right)=\left(\begin{array}{cc}
\mathcal{M}_{n}(t) & U a_{n}^{(0) 2} \\
-U a_{n}^{(0) * 2} & -\mathcal{M}_{n}(t)^{*}
\end{array}\right)\left(\begin{array}{c}
\delta a_{n} \\
\delta a_{n}^{*}
\end{array}\right),
$$

where we introduced the notation

$$
\begin{aligned}
\mathcal{M}_{n}(t) \delta a_{n} \equiv & -J\left(\delta a_{n+1}+\delta a_{n-1}\right)+2 U\left|a_{n}^{(0)}\right|^{2} \\
& +K \cos (\omega t) n \delta a_{n} .
\end{aligned}
$$

The resulting form of the Bogoliubov equations in Eq. (A2), which features spurious complex time-dependent off-diagonal terms, is quite unusual and, in fact, not very convenient. First of all, we find that those terms can lead to numerical instabilities, when solving this equation using a finite-difference scheme. Then, we note that the form (A2) does not allow for a simple comparison with the standard static case: Indeed, setting $K=0$ and writing $a_{n}^{(0)}(t)=\sqrt{\rho} e^{-i \mu t}$, with $\rho$ the condensate density and $\mu=$ $g-2 J$ the chemical potential (and $g \equiv U \rho$ ), we find that Eq. (A2) yields the nonstandard form

$$
i\left(\begin{array}{c}
\delta \dot{a}_{n} \\
\dot{\delta} a_{n}^{*}
\end{array}\right)=\left(\begin{array}{cc}
-J \hat{L}+2 g & g e^{-2 i \mu t} \\
g e^{2 i \mu t} & J \hat{L}-2 g
\end{array}\right)\left(\begin{array}{c}
\delta a_{n} \\
\delta a_{n}^{*}
\end{array}\right),
$$

where the discrete operator $\hat{L}$ is defined by $\hat{L}(\cdot)_{n} \equiv$ $(\cdot)_{n+1}+(\cdot)_{n-1}$. The equations of motion (A4) are still explicitly time dependent, and hence, they do not allow for a direct identification of the quasiparticle spectrum.

In the static case, a common way of solving this issue is to use a slightly different parametrization, namely, $a_{n}(t)=$ $e^{-i \mu t}\left[a_{n}^{(0)}(t)+\delta a_{n}(t)\right]$, where we have factored out the dynamical phase $\mu$ associated with the free evolution. Using this parametrization, we obtain the Bogoliubov equations in the form

$$
i\left(\begin{array}{c}
\dot{\delta} a_{n} \\
\dot{\delta a_{n}^{*}}
\end{array}\right)=\left(\begin{array}{cc}
-J \hat{\Delta}+g & g \\
g & J \hat{\Delta}-g
\end{array}\right)\left(\begin{array}{l}
\delta a_{n} \\
\delta a_{n}^{*}
\end{array}\right),
$$

where $\hat{\Delta} \delta a_{n} \equiv \delta a_{n+1}-2 \delta a_{n}+\delta a_{n-1}$ denotes the discrete Laplacian. The resulting equations of motion correspond to the standard form of the BdG equations, which are indeed time independent (the spurious complex off-diagonal terms have disappeared), allowing for a direct extraction of the Bogoliubov spectrum.

In the driven-system situation, the time-dependent phase of the BEC wave function $a_{n}^{(0)}(t)$ does not reduce to a trivial phase $\mu$, as it also contains an additional dynamical phase induced by the periodic driving. Therefore, factorizing $e^{-i \mu t}$ alone in the Bogoliubov parametrization would not be sufficient to get rid of all the spurious terms. In order to solve that issue, we generalize the method discussed above by factorizing the entire time-dependent phase of $a_{n}^{(0)}(t)$ : This is achieved by writing 


$$
a_{n}(t)=e^{i \phi_{n}(t)}\left[\rho_{n}(t)+\delta a_{n}(t)\right],
$$

where we have introduced the modulus and phase of the BEC wave function, $a_{n}^{(0)}(t)=\rho_{n}(t) e^{i \phi_{n}(t)}$.

For the model considered in this work, translational invariance is recovered in the rotating frame, which allows one to further simplify the parametrization: Indeed, taking into account the fact that $\rho_{n}(t)$ is uniform (it does not depend on $n$ ), we can equivalently write Eq. (A6) in the form [Eq. (4)]

$$
a_{n}(t)=a_{n}^{(0)}(t)\left[1+\delta a_{n}(t)\right]
$$

where we have now factorized the full condensate wave function. Linearizing the Gross-Pitaevskii equation in $\delta a_{n}$ then yields the time-dependent Bogoliubov-de Gennes equations, which take the general form considered in the main text,

$$
i\left(\begin{array}{c}
\dot{\delta} \dot{a}_{n} \\
\dot{\delta} a_{n}^{*}
\end{array}\right)=\left(\begin{array}{cc}
\mathcal{F}_{n}(t) & U\left|a_{n}^{(0)}\right|^{2} \\
-U\left|a_{n}^{(0)}\right|^{2} & -\mathcal{F}_{n}(t)^{*}
\end{array}\right)\left(\begin{array}{c}
\delta a_{n} \\
\delta a_{n}^{*}
\end{array}\right),
$$

where we introduced the operator $\mathcal{F}_{n}(t)$, whose action on $\delta a_{n}$ is defined by

$$
\begin{aligned}
\mathcal{F}_{n}(t) \delta a_{n} \equiv & -J \frac{\hat{L}\left(a^{(0)} \delta a_{n}\right)_{n}}{a_{n}^{(0)}}+2 U\left|a_{n}^{(0)}\right|^{2} \delta a_{n} \\
& +K \cos (\omega t) n \delta a_{n}-i \frac{\dot{a}_{n}^{(0)}}{a_{n}^{(0)}} \delta a_{n} .
\end{aligned}
$$

Compared to Eq. (A2), the differences are twofold. First of all, the off-diagonal terms are real in this approach,

which is numerically more practical. Then, we note that the last term in Eq. (A9), which involves the logarithmic derivative of the BEC wave function $a_{n}^{(0)}(t)$, features a time-dependent chemical potential, which takes into account the entire dynamical phase induced by the periodic driving; in particular, we recover the chemical potential term $-\mu=-g+2 J$ in the static limit $K \rightarrow 0$.

For the reasons detailed above, we have chosen to consider the convenient parametrization defined in Eq. (A7), both in our numerical and in our analytical extraction of the instability rates; see also Eqs. (47) for the extension to WCCA. We stress that the parametrization in Eq. (A7) should be revised for more elaborate models (multiband, multicell,...), where $\rho_{n}(t)$ is not uniform, using the more general form (A6). Finally, we emphasize that all parametrizations lead to the same physical results and that choosing one or the other is just a matter of technical convenience.

\section{APPENDIX B: USEFUL RESULTS ON THE PARAMETRIC OSCILLATOR}

The simplest parametric oscillator is the system described by the equation of motion

$$
\ddot{x}(t)+\omega_{0}^{2}[1+\alpha \cos (\omega t)] x(t)=0,
$$

describing a harmonic oscillator of pulsation $\omega_{0}$ perturbed by a sinusoidal modulation of pulsation $\omega$ and amplitude $\alpha \ll 1$. Equivalently, one can introduce the standard operators $\gamma, \gamma^{\dagger}$ defined by $x=\left(\gamma+\gamma^{\dagger}\right) / \sqrt{2 \omega_{0}}$ and $p=$ $i \sqrt{\omega_{0} / 2}\left(\gamma^{\dagger}-\gamma\right)$, and use the parametrization $\gamma(t)=$ $\tilde{u}^{\prime}(t) \gamma(t=0)-\tilde{v}^{\prime *}(t) \gamma^{\dagger}(t=0)$ to rewrite the equation of motion (B1) in the following way (see Ref. [74] for details):

$$
i \partial_{t}\left(\begin{array}{c}
\tilde{u}^{\prime}(t) \\
\tilde{v}^{\prime}(t)
\end{array}\right)=\left[\omega_{0} \hat{\mathbf{1}}+W(t) \hat{\sigma}_{z}+\frac{\alpha \omega_{0}}{2}\left(\begin{array}{cc}
0 & \cos (\omega t) e^{-2 i \omega_{0} t} \\
-\cos (\omega t) e^{2 i \omega_{0} t} & 0
\end{array}\right)\right]\left(\begin{array}{c}
\tilde{u}^{\prime}(t) \\
\tilde{v}^{\prime}(t)
\end{array}\right),
$$

where $W(t)=\alpha \omega_{0} / 2 \cos (\omega t)$. As mentioned in Sec. IVA, the simplest way to identify the appearance of parametric resonance in this model is to perform a RWA treatment of Eq. (B2). Since the latter consists in taking the time average over a drive period in Eq. (B2), the zero-average term $W(t) \hat{\sigma}_{z}$ does not contribute, and a pronounced contribution to the dynamics is found when $\omega=2 \omega_{0}$. Keeping the corresponding resonant terms only yields a timeindependent $2 \times 2$ matrix in Eq. (B2), which can straightforwardly be diagonalized. Its eigenvalues then exhibit an imaginary part responsible for an exponential growth of the solution. The instability rate (here, on resonance) $s^{(R)}$ is thus given by the imaginary part of the eigenvalues of this $2 \times 2$ matrix, yielding $s^{(R)}=\alpha \omega_{0} / 4$. This corresponds to the rate on resonance, i.e., precisely when $\omega=2 \omega_{0}$ [113].
However, a key feature that will have crucial importance in the following is that instability in fact arises not only when the resonance condition is precisely satisfied but in a full range of parameters around the resonance point. To investigate more precisely how the rate behaves in the vicinity of the resonance, and at which point it will eventually drop to zero (which will give the limits of the instability domain), one can follow the general procedure indicated in Landau and Lifshits [3]. The idea is to write $\omega=2 \omega_{0}+\delta$ and to solve the problem perturbatively in the detuning $\delta$ and $\alpha$ [114]. At lowest order, we seek a solution of Eq. (B1) of the form $x(t)=A(t) \cos \left[\left(\omega_{0}+\delta / 2\right) t\right]+$ $B(t) \sin \left[\left(\omega_{0}+\delta / 2\right) t\right]$, where $A(t)$ and $B(t)$ vary slowly compared to the oscillating terms. Inserting this into Eq. (B1) and solving it to first order in $\delta$ eventually 
yields that $A(t)$ and $B(t)$ behave as $e^{s t}$, with a rate $s$ given by

$$
s=s^{(R)} \sqrt{1-\left(2 \delta / \alpha \omega_{0}\right)^{2}} ; \quad s^{(R)}=\alpha \omega_{0} / 4 .
$$

At first order, the instability rate is thus maximal on resonance and decreases around it, and instability arises for all values

$$
\omega \in\left[2 \omega_{0}-\alpha \omega_{0} / 2 ; 2 \omega_{0}+\alpha \omega_{0} / 2\right] .
$$

This calculation can be extended to higher orders by adding in the ansatz for the solution harmonics that will be of higher order in the detuning. For instance, at second order, the idea is to seek a solution of the form

$$
\begin{aligned}
x(t)= & A_{0}(t) \cos \left[\left(\omega_{0}+\delta / 2\right) t\right]+B_{0}(t) \sin \left[\left(\omega_{0}+\delta / 2\right) t\right] \\
& +A_{1}(t) \cos \left[3\left(\omega_{0}+\delta / 2\right) t\right]+B_{1}(t) \sin \left[3\left(\omega_{0}+\delta / 2\right) t\right]
\end{aligned}
$$

and to solve Eq. (B1) to second order in $\delta$ and $\alpha$. We find an unstable regime for

$\omega \in\left[2 \omega_{0}-\alpha \omega_{0} / 2-\alpha^{2} \omega_{0} / 32 ; 2 \omega_{0}+\alpha \omega_{0} / 2-\alpha^{2} \omega_{0} / 32\right]$,

while the instability rate $s$ is the solution of the implicit equation

$$
\left|\begin{array}{cccc}
s^{2}+\omega_{0}^{2}-\omega^{2} / 4+\alpha \omega_{0}^{2} / 2 & \omega s & \alpha \omega_{0}^{2} / 2 & 0 \\
-\omega s & s^{2}+\omega_{0}^{2}-\omega^{2} / 4-\alpha \omega_{0}^{2} / 2 & 0 & \alpha \omega_{0}^{2} / 2 \\
\alpha \omega_{0}^{2} / 2 & 0 & s^{2}-8 \omega_{0}^{2} & 3 \omega s \\
0 & \alpha \omega_{0}^{2} / 2 & -3 \omega s & s^{2}-8 \omega_{0}^{2}
\end{array}\right|=0,
$$

which can be numerically solved. Similarly to the firstorder result, the instability rate continuously decreases from its maximal value to zero at the edges of the instability domain; yet, the latter is no longer centered on the resonance point but is slightly shifted; therefore, the maximal instability rate, which is reached at the center of the instability domain, does not coincide with the resonance point (although it is not far from it). We conclude this appendix by pointing out that the rates are independent with respect to the time-dependent term $W(t) \hat{\sigma}_{z}$, at all orders of this perturbative treatment.

\section{APPENDIX C: CHANGE OF BASIS LEADING TO EQ. (19)}

In this appendix, we describe the change of basis introduced in Eq. (19). Starting from the Bogoliubov equations written in Fourier space [Eq. (9)],

$i \partial_{t}\left(\begin{array}{l}u_{q} \\ v_{q}\end{array}\right)=\left(\begin{array}{cc}\varepsilon(q, t)+g & g \\ -g & -\varepsilon(-q, t)-g\end{array}\right)\left(\begin{array}{l}u_{q} \\ v_{q}\end{array}\right)$,

where

$$
\varepsilon(q, t)=4 J \sin \left(\frac{q}{2}\right) \sin \left(\frac{q}{2}+p_{0}-\frac{K}{\omega} \sin (\omega t)\right),
$$

one first introduces the change of basis that diagonalizes the time-averaged part of the equation,

$$
\left(\begin{array}{c}
u_{q} \\
v_{q}
\end{array}\right)=\left(\begin{array}{cc}
\cosh \left(\theta_{q}\right) & \sinh \left(\theta_{q}\right) \\
\sinh \left(\theta_{q}\right) & \cosh \left(\theta_{q}\right)
\end{array}\right)\left(\begin{array}{c}
u_{q}^{\prime} \\
v_{q}^{\prime}
\end{array}\right)
$$

where $\cosh \left(2 \theta_{q}\right) \equiv\left(4\left|J_{\text {eff }}\right| \sin ^{2}(q / 2)+g\right) / E_{\text {av }}(q)$ and $\sinh \left(2 \theta_{q}\right) \equiv g / E_{\text {av }}(q)$, with

$$
E_{\mathrm{av}}(q)=\sqrt{4\left|J_{\mathrm{eff}}\right| \sin ^{2}(q / 2)\left(4\left|J_{\mathrm{eff}}\right| \sin ^{2}(q / 2)+2 g\right)}
$$

the time-averaged Bogoliubov dispersion. Then, defining a second transformation

$$
\left(\begin{array}{c}
\tilde{u}_{q}^{\prime} \\
\tilde{v}_{q}^{\prime}
\end{array}\right)=\left(\begin{array}{cc}
e^{2 i E_{\mathrm{av}}(q) t} & 0 \\
0 & 1
\end{array}\right)\left(\begin{array}{c}
u_{q}^{\prime} \\
v_{q}^{\prime}
\end{array}\right)
$$

the Bogoliubov equations take the final form

$$
i \partial_{t}\left(\begin{array}{c}
\tilde{u}_{q}^{\prime} \\
\tilde{v}_{q}^{\prime}
\end{array}\right)=\left[E_{\mathrm{av}}(q) \hat{\mathbf{1}}+\hat{W}_{q}(t)+\sinh \left(2 \theta_{q}\right)\left(\begin{array}{cc}
0 & h_{q}(t) e^{-2 i E_{\mathrm{av}}(q) t} \\
-h_{q}(t) e^{2 i E_{\mathrm{av}}(q) t} & 0
\end{array}\right)\right]\left(\begin{array}{c}
\tilde{u}_{q}^{\prime} \\
\tilde{v}_{q}^{\prime}
\end{array}\right),
$$

where $\sinh \left(2 \theta_{q}\right)=g / E_{\mathrm{av}}(q), E_{\mathrm{av}}(q)$ is the Bogoliubov dispersion associated with the effective GPE within the Bogoliubov approximation [see Eq. (12)], $\hat{W}_{q}(t)$ is a diagonal matrix of zero average over one driving period, 


$$
\begin{aligned}
\hat{W}_{q}(t)= & 4 J \sin (q / 2) \sum_{l \neq 0} \mathcal{J}_{l}(K / \omega) \\
& \times\left(\begin{array}{cc}
\cosh ^{2} \theta_{q} \sin \left(\frac{q}{2}-l \omega t\right)+\sinh ^{2} \theta_{q} \sin \left(\frac{q}{2}+l \omega t\right) & 0 \\
0 & -\sinh ^{2} \theta_{q} \sin \left(\frac{q}{2}-l \omega t\right)+\cosh ^{2} \theta_{q} \sin \left(\frac{q}{2}+l \omega t\right)
\end{array}\right),
\end{aligned}
$$

and $h_{q}(t)$ is a (real-valued) function that can be Fourier expanded as

$$
\begin{aligned}
h_{q}(t) & =\frac{J}{2} 4 \sin ^{2}(q / 2) \sum_{l=-\infty}^{\infty}\left[\mathcal{J}_{l}(K / \omega)+\mathcal{J}_{-l}(K / \omega)\right] \mathrm{e}^{i l \omega t} \\
& =4 J \sin ^{2}(q / 2) \sum_{l=-\infty}^{\infty} \mathcal{J}_{2 l}(K / \omega) \mathrm{e}^{i 2 l \omega t}
\end{aligned}
$$

with $\mathcal{J}_{l}(z)$ the $l$ th Bessel function of the first kind.

Because of the formal similarity with Eq. (B2), the instability rates associated with the equations of motion (C4) can be directly evaluated, following the treatment described in Appendix B. In particular, it is therefore apparent that the time-dependent term $\hat{W}_{q}(t)$ does not contribute to these extracted rates, highlighting the convenience of the specific frame (gauge) considered in this analysis. We recall that, while the short-time behavior (micromotion) of physical observables generally depends on the chosen frame, the instability rates defined in Sec. II, which are associated with the long-time (stroboscopic) dynamics, are independent of this choice (see Figs. 4 and 6 for an illustration of the distinction between the micromotion and long-time behaviors).

\section{APPENDIX D: DISAGREEMENT REGIONS}

The analytical approach is based on a perturbative solution in the detuning and the amplitude of the perturbation, which is performed on an effective model restricted to the second harmonic (see Sec. IV B). We observe that it breaks down into two main regions:

(i) The main discrepancy with the numerical results occurs around the first zero of $\mathcal{J}_{0}$ (the only one where $\mathcal{J}_{2}$ is not small as well, zones $\mathrm{C}$ in Fig. 2), the analytics fails to capture the numerically observed instability, and we also notice a completely different behavior between the first-order and second-order analytics (see Fig. 20). This comes from the fact that, in this case, the effective dispersion $E_{\mathrm{av}}$ is flat, and the perturbative approach breaks down. More precisely, the first-order analytical solution gives a resonance domain centered on $\omega=E_{\text {av }}(q) \approx 0$ but of infinite width $\left[\propto E_{\mathrm{av}}(q)^{-1}\right]$, thus displaying a strong instability. The second-order correction displaces the center of the resonance domain as $\propto E_{\text {av }}(q)^{-3}$, sweeping away all instability in the considered parameter range. More generally, the perturbative approach does not converge and cannot capture the observed behavior (which seems to be due to the second harmonic alone).

(ii) Slight discrepancies also occur "between" the lobes of the stability diagram, as the numerics and the analytics disagree on the way those "black zones" close (or not) at large $g$. First, near the zeros of $\mathcal{J}_{0}$ (i.e., at the left border of each lobe, zones $\mathrm{D}$ in Fig. 2), the numerics displays a transition to instability which is not captured by the analytics (similarly to what was observed in case C). However, this failure of the analytics is less marked than in case $\mathrm{C}$ (in the sense that it appears for much larger $g$ ) for two main reasons: On the one hand, the fact that $\mathcal{J}_{2}(K / \omega)$ is also small can partly kill the divergences due to the vanishing of $\mathcal{J}_{0}(K / \omega)$; on the other hand, the stable regime extends here to much larger $g$, coherently with the fact that the second harmonic has a very small amplitude [indeed, as visible in Fig. 21(a), the numerical instability seems rather to be from higher harmonics). More precisely, at points where $\mathcal{J}_{2}(K / \omega)=0$, the analytics, which is essentially built on a second-harmonic-based model, is always stable and cannot reproduce the numerical instabilities due to higher harmonics (which nevertheless show up only at large $g$ ). Near such points

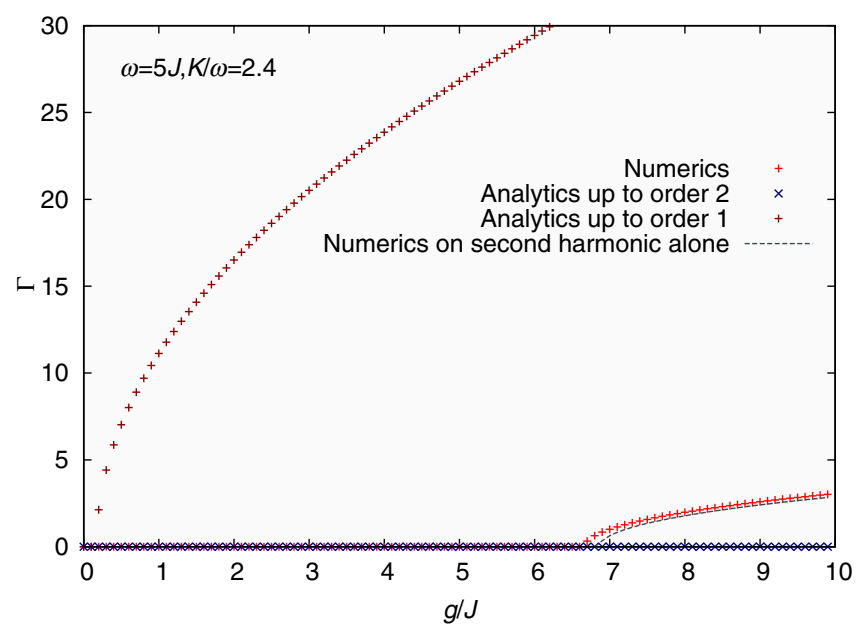

FIG. 20. Comparison between the numerically and analytically computed instability rate $\Gamma$ in the case $K / \omega=2.4$ [zone $\mathrm{C}$ on the stability diagram of Fig. 2, where $\mathcal{J}_{0}(K / \omega) \approx 0$ and $\mathcal{J}_{2}(K / \omega)$ is large]. Since the effective Bogoliubov dispersion is flat, the analytical perturbative approach breaks down. 


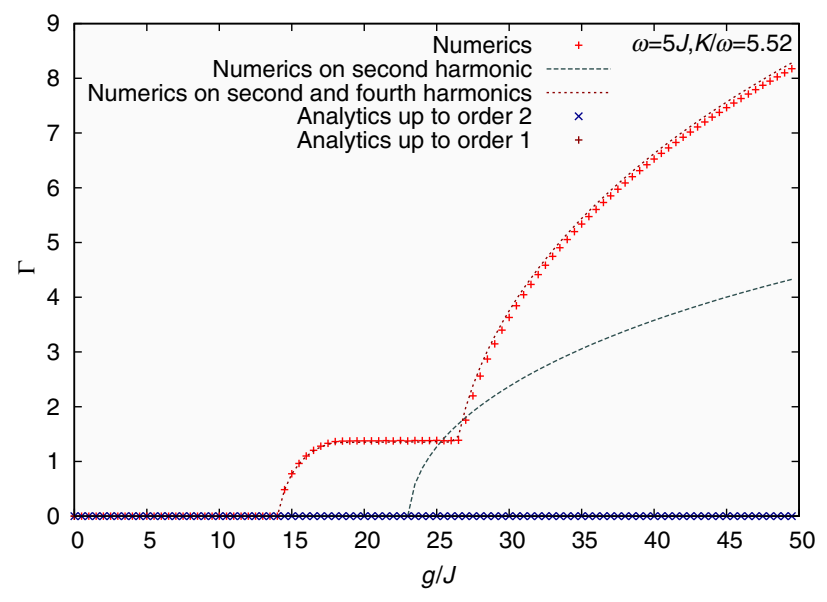

(a)

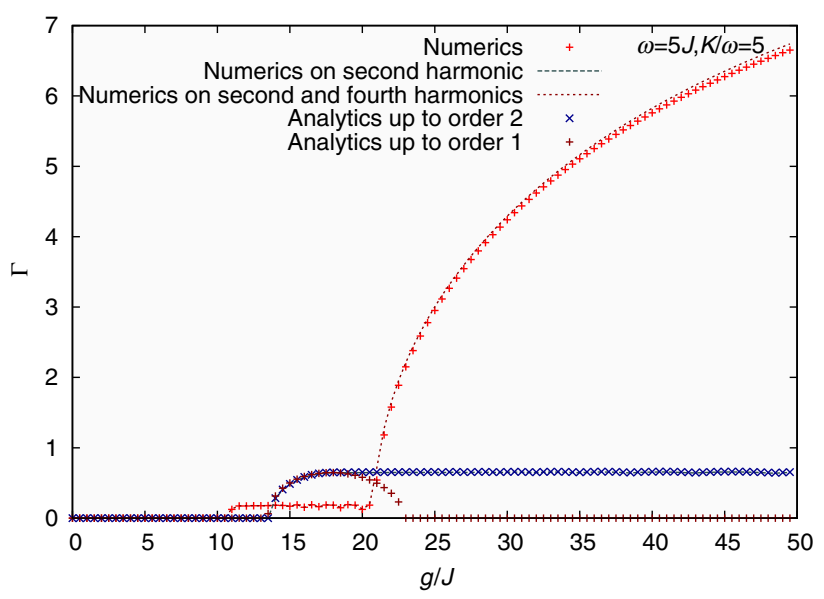

(b)

FIG. 21. Comparison between the numerically and analytically computed instability rate $\Gamma$ in the cases $K / \omega=5.52$ [(left panel), zone D of the stability diagram of Fig. 2, where $\mathcal{J}_{0}(K / \omega) \approx 0$ ] and $K / \omega=5$ [(right panel), zone E of the stability diagram of Fig. 2, where $\mathcal{J}_{2}(K / \omega) \approx 0$ ]. The disagreement in those cases can be due to a breakdown of the perturbative approach [when $\mathcal{J}_{0}(K / \omega)$ vanishes, $($ left panel)] and/or a vanishing of the second harmonic [when $\mathcal{J}_{2}(K / \omega)$ vanishes, (right panel)].

(zones E), the numerical instability seems to be due to a joined effect of the second and next harmonics, and it is therefore not accurately captured by the analytical approach [see Fig. 21(b)].

[1] G. Casati, B. V. Chirikov, F. M. Izrailev, and J. Ford, Stochastic Behavior of a Quantum Pendulum under a Periodic Perturbation, Lecture Notes Phys. 93, 334 (1979).

[2] P. Kapitza, Dynamical Stability of a Pendulum When its Point of Suspension Vibrates, Sov. Phys. JETP 21, 588 (1951).

[3] L. Landau and E. Lifshits, Theoretical Physics (Pergamon Press, Oxford, 1969), Vol. 1.

[4] D. Leibfried, R. Blatt, C. Monroe, and D. Wineland, Quantum Dynamics of Single Trapped Ions, Rev. Mod. Phys. 75, 281 (2003).

[5] L. Lu, J. D. Joannopoulos, and M. Soljačić, Topological Photonics, Nat. Photon 8, 821 (2014).

[6] J. Cayssol, B. Dora, F. Simon, and R. Moessner, Floquet Topological Insulators, J. Phys. Status Solidi Rapid Res. Lett. 7, 101 (2013).

[7] T. Oka and H. Aoki, Photovoltaic Hall Effect in Graphene, Phys. Rev. B 79, 081406 (2009).

[8] T. Kitagawa, T. Oka, A. Brataas, L. Fu, and E. Demler, Transport Properties of Nonequilibrium Systems under the Application of Light: Photoinduced Quantum Hall Insulators without Landau Levels, Phys. Rev. B 84, 235108 (2011).

[9] N. Goldman, J. C. Budich, and P. Zoller, Topological Quantum Matter with Ultracold Gases in Optical Lattices, Nat. Phys. 12, 639 (2016).

[10] A. Eckardt, Atomic Quantum Gases in Periodically Driven Optical Lattices, arXiv:1606.08041v1 [Rev. Mod. Phys. (to be published)].
[11] N. Goldman and J. Dalibard, Periodically-Driven Quantum Systems: Effective Hamiltonians and Engineered Gauge Fields, Phys. Rev. X 4, 031027 (2014).

[12] M. Bukov, L. D’Alessio, and A. Polkovnikov, Universal High-Frequency Behaviour of Periodically-Driven Quantum Systems: From Dynamical Stabilization to Floquet Engineering, Adv. Phys. 64, 139 (2015).

[13] J. H. Shirley, Solution of the Schrodinger Equation with a Hamiltonian Periodic in Time, Phys. Rev. 138B, B979 (1965).

[14] S. Rahav, I. Gilary, and S. Fishman, Effective Hamiltonians for Periodically Driven Systems, Phys. Rev. A 68, 013820 (2003).

[15] This change of basis is important to keep in mind when preparing the system in a given initial state.

[16] N. Goldman, J. Dalibard, M. Aidelsburger, and N. Cooper, Periodically-Driven Quantum Matter: The Case of Resonant Modulations, Phys. Rev. A 91, 033632 (2015).

[17] A. Eckardt and E. Anisimovas, High-Frequency Approximation for Periodically-Driven Quantum Systems from a Floquet-Space Perspective, New J. Phys. 17, 093039 (2015).

[18] T. Mikami, S. Kitamura, K. Yasuda, N. Tsuji, T. Oka, and H. Aoki, Brillouin-Wigner Theory for High-Frequency Expansion in Periodically Driven Systems: Application to Floquet Topological Insulators, Phys. Rev. B 93, 144307 (2016).

[19] S. Rahav, I. Gilary, and S. Fishman, Time Independent Description of Rapidly Oscillating Potentials, Phys. Rev. Lett. 91, 110404 (2003).

[20] S. Blanes, F. Casas, J. A. Oteo, and J. Ros, The Magnus Expansion and Some of Its Applications, Phys. Rep. 470, 151 (2009).

[21] I. M. Georgescu, S. Ashhab, and F. Nori, Quantum Simulation, Rev. Mod. Phys. 86, 153 (2014).

[22] D. H. Dunlap and V. M. Kenkre, Dynamic Localization of a Charged Particle Moving under the Influence of an Electric Field, Phys. Rev. B 34, 3625 (1986). 
[23] D. H. Dunlap and V. M. Kenkre, Effect of Scattering on the Dynamic Localization of a Particle in a Time-Dependent Electric Field, Phys. Rev. B 37, 6622 (1988).

[24] M. Holthaus, Collapse of Minibands in Far-Infrared Irradiated Superlattices, Phys. Rev. Lett. 69, 351 (1992).

[25] H. Lignier, C. Sias, D. Ciampini, Y. Singh, A. Zenesini, O. Morsch, and E. Arimondo, Dynamical Control of MatterWave Tunneling in Periodic Potentials, Phys. Rev. Lett. 99, 220403 (2007).

[26] A. Eckardt, M. Holthaus, H. Lignier, A. Zenesini, D. Ciampini, O. Morsch, and E. Arimondo, Exploring Dynamic Localization with a Bose-Einstein Condensate, Phys. Rev. A 79, 013611 (2009).

[27] A. Eckardt and M. Holthaus, AC-Induced Superfluidity, Europhys. Lett. 80, 50004 (2007).

[28] C. Sias, H. Lignier, Y. Singh, A. Zenesini, D. Ciampini, O. Morsch, and E. Arimondo, Observation of PhotonAssisted Tunneling in Optical Lattices, Phys. Rev. Lett. 100, 040404 (2008).

[29] A. Eckardt, C. Weiss, and M. Holthaus, SuperfluidInsulator Transition in a Periodically-Driven Optical Lattice, Phys. Rev. Lett. 95, 260404 (2005).

[30] D. Ciampini, O. Morsch, and E. Arimondo, Quantum Control in Strongly Driven Optical Lattices, Int. J. Quantum. Inform. 09, 139 (2011).

[31] A. Zenesini, H. Lignier, D. Ciampini, O. Morsch, and E. Arimondo, Coherent Control of Dressed Matter Waves, Phys. Rev. Lett. 102, 100403 (2009).

[32] A. Zenesini, H. Lignier, O. Morsch, D. Ciampini, and E. Arimondo, Tunneling Control and Localization for BoseEinstein Condensates in a Frequency Modulated Optical Lattice, Laser Phys. 20, 1182 (2010).

[33] S. Fishman, D. R. Grempel, and R. E. Prange, Chaos, Quantum Recurrences and Anderson Localization, Phys. Rev. Lett. 49, 509 (1982).

[34] G. Casati, I. Guarneri, and D. L. Shepelyansky, Anderson Transition in a One-Dimensional System with Three Incommensurate Frequencies, Phys. Rev. Lett. 62, 345 (1989).

[35] M. Lopez, J. F. Clément, P. Szriftgiser, J. C. Garreau, and D. Delande, Experimental Test of Universality of the Anderson Transition, Phys. Rev. Lett. 108, 095701 (2012).

[36] M. Aidelsburger, M. Atala, S. Nascimbene, S. Trotzky, Y. Chen, and I. Bloch, Experimental Realization of Strong Effective Magnetic Fields in an Optical Lattice, Phys. Rev. Lett. 107, 255301 (2011).

[37] M. Aidelsburger, M. Atala, M. Lohse, J. Barreiro, B. Paredes, and I. Bloch, Realization of the Hofstadter Hamiltonian with Ultracold Atoms in Optical Lattices, Phys. Rev. Lett. 111, 185301 (2013).

[38] H. Miyake, G. A. Siviloglou, C. J. Kennedy, W. C. Burton, and W. Ketterle, Realizing the Harper Hamiltonian with Laser-Assisted Tunneling in Optical Lattices, Phys. Rev. Lett. 111, 185302 (2013).

[39] M. Aidelsburger, M. Lohse, C. Schweizer, M. Atala, J. Barreiro, S. Nascimbene, N. R. Cooper, I. Bloch, and N. Goldman, Measuring the Chern Number of Hofstadter Bands with Ultracold Bosonic Atoms, Nat. Phys. 11, 162 (2015).
[40] G. Jotzu, M. Messer, R. Desbuquois, M. Lebrat, T. Uehlinger, D. Greif, and T. Esslinger, Experimental Realisation of the Topological Haldane Model, Nature (London) 515, 237 (2014).

[41] C. J. Kennedy, W. C. Burton, W. C. Chung, and W. Ketterle, Observation of Bose-Einstein Condensation in a Strong Synthetic Magnetic Field, Nat. Phys. 11, 859 (2015).

[42] C. E. Creffield, G. Pieplow, F. Sols, and N. Goldman, Realization of Uniform Synthetic Magnetic Fields by Periodically Shaking an Optical Square Lattice, New J. Phys. 18, 093013 (2016).

[43] A. Eckardt, P. Hauke, P. Soltan-Panahi, C. Becker, K. Sengtock, and M. Lewenstein, Frustrated Quantum Antiferromagnetism with Ultracold Bosons in a Triangular Lattice, Europhys. Lett. 89, 10010 (2010).

[44] J. Struck, C. Olschlager, R. Le Targat, P. Soltan-Panahi, A. Eckardt, M. Lewenstein, P. Windpassinger, and K. Sengstock, Quantum Simulation of Frustrated Classical Magnetism in Triangular Optical Lattices, Science 333, 996 (2011).

[45] R. Desbuquois, M. Messer, F. Görg, K. Sandholzer, G. Jotzu, and T. Esslinger, Controlling the Floquet State Population and Observing Micromotion in a Periodically Driven Two-Body Quantum System, arXiv:1703.07767 [Phys. Rev X (to be published)].

[46] G. Jotzu, M. Messer, F. Görg, D. Greif, R. Desbuquois, and T. Esslinger, Creating State-Dependent Lattices for Ultracold Fermions by Magnetic Gradient Modulation, Phys. Rev. Lett. 115, 073002 (2015).

[47] N. Fläschner, D. Vogel, M. Tarnowski, B. S. Rem, D. S. Lühmann, M. Heyl, J. C. Budich, L. Mathey, K. Sengstock, and C. Weitenberg, Observation of a Dynamical Topological Phase Transition, arXiv:1608:05616.

[48] A. Robertson, V. M. Galitski, and G. Refael, Dynamic Stimulation of Quantum Coherence in Systems of Lattice Bosons, Phys. Rev. Lett. 106, 165701 (2011).

[49] S. Nascimbène, N. Goldman, N. R. Cooper, and J. Dalibard, Dynamic Optical Lattices of Sub-wavelength Spacing for Ultracold Atoms, Phys. Rev. Lett. 115, 140401 (2015).

[50] H. M. Price, T. Ozawa, and N. Goldman, Synthetic Dimensions for Cold Atoms from Shaking a Harmonic Trap, Phys. Rev. A 95, 023607 (2017).

[51] J. C. Budich, Y. Hu, and P. Zoller, Helical Floquet Channels in $1 D$ Lattices, Phys. Rev. Lett. 118, 105302 (2017).

[52] G. Goldstein, C. Aron, and C. Chamon, Photo-Induced Superconductivity in Semiconductors, Phys. Rev. B 91, 054517 (2015).

[53] D. Fausti, R. I. Tobey, N. Dean, S. Kaiser, A. Dienst, M. C. Hoffmann, S. Pyon, T. Takayama, H. Takagi, and A. Cavalleri, Light-Induced Superconductivity in a StripeOrdered Cuprate, Science 331, 189 (2011).

[54] M. Knap, M. Babadi, I. Martin, and E. Demler, Dynamical Cooper Pairing in Non-equilibrium Electron-Phonon Systems, Phys. Rev. B 94, 214504 (2016).

[55] N. H. Lindner, G. Refael, and V. Galitski, Floquet Topological Insulator in Semiconductor Quantum Wells, Nat. Phys. 7, 490 (2011). 
[56] K. Sacha, Modeling Spontaneous Breaking of TimeTranslation Symmetry, Phys. Rev. A 91, 033617 (2015).

[57] D. V. Else, B. Bauer, and C. Nayak, Floquet Time Crystals, Phys. Rev. Lett. 117, 090402 (2016).

[58] J. Zhang, P. W. Hess, A. Kyprianidis, P. Becker, A. Lee, J. Smith, G. Pagano, I. D. Potirniche, A. C. Potter, A. Vishwanath, N. Y. Yao, and C. Monroe, Observation of a Discrete Time Crystal, arXiv:1609.08684.

[59] E. Anisimovas, G. Zlabys, B. M. Anderson, G. Juzeliunas, and A. Eckardt, The Role of Real-Space Micromotion for Bosonic and Fermionic Floquet Fractional Chern Insulators, Phys. Rev. B 91, 245135 (2015).

[60] A. G. Grushin, A. Gómez-León, and T. Neupert, Floquet Fractional Chern Insulators, Phys. Rev. Lett. 112, 156801 (2014).

[61] M. Bukov, M. Kolodrubetz, and A. Polkovnikov, Schrieffer-Wolff Transformation for Periodically Driven Systems: Strongly Correlated Systems with Artificial Gauge Fields, Phys. Rev. Lett. 116, 125301 (2016).

[62] P. Weinberg, M. Bukov, S. Vajna, L. D’Alessio, A. Polkovnikov, and M. Kolodrubetz, Adiabatic Perturbation Theory and Geometry of Periodically-Driven Systems, arXiv:1606.02229.

[63] T. Bilitewski and N. R. Cooper, Population Dynamics in a Floquet Realization of the Harper-Hofstadter Hamiltonian, Phys. Rev. A 91, 063611 (2015).

[64] T. Bilitewski and N. R. Cooper, Scattering Theory for Floquet-Bloch States, Phys. Rev. A 91, 033601 (2015).

[65] S. Choudhury and E. Mueller, Stability of a Floquet BoseEinstein Condensate in a One-Dimensional Optical Lattice, Phys. Rev. A 90, 013621 (2014).

[66] S. Choudhury and E. Mueller, Transverse Collisional Instabilities of a Bose-Einstein Condensate in a Driven One-Dimensional Lattice, Phys. Rev. A 91, 023624 (2015).

[67] L. Fallani, L. De Sarlo, J. E. Lye, M. Modugno, R. Saers, C. Fort, and M. Inguscio, Observation of Dynamical Instability for a Bose-Einstein Condensate in a Moving 1D Optical Lattice, Phys. Rev. Lett. 93, 140406 (2004).

[68] M. Modugno, C. Tozzo, and F. Dalfovo, Role of Transverse Excitations in the Instability of Bose-Einstein Condensates Moving in Optical Lattices, Phys. Rev. A 70, 043625 (2004).

[69] Y. Zheng, M. Kostrun, and J. Javanainen, Low-Acceleration Instability of a Bose-Einstein Condensate in an Optical Lattice, Phys. Rev. Lett. 93, 230401 (2004).

[70] M. Kramer, C. Tozzo, and F. Dalfovo, Parametric Excitation of a Bose-Einstein Condensate in a One-Dimensional Optical Lattice, Phys. Rev. A 71, 061602 (2005).

[71] C. Tozzo, M. Kramer, and F. Dalfovo, Stability Diagram and Growth Rate of Parametric Resonances in BoseEinstein Condensates in One-Dimensional Optical Lattices, Phys. Rev. A 72, 023613 (2005).

[72] L. De Sarlo, L. Fallani, J. E. Lye, M. Modugno, R. Saers, C. Fort, and M. Inguscio, Unstable Regimes for a BoseEinstein Condensate in an Optical Lattice, Phys. Rev. A 72, 013603 (2005).

[73] C. E. Creffield, Instability and Control of a PeriodicallyDriven Bose-Einstein Condensate, Phys. Rev. A 79, 063612 (2009).
[74] M. Bukov, S. Gopalakrishnan, M. Knap, and E. Demler, Prethermal Floquet Steady States and Instabilities in the Periodically Driven, Weakly Interacting Bose-Hubbard Model, Phys. Rev. Lett. 115, 205301 (2015).

[75] R. Citro, E. G. Dalla Torre, L. D’Alessio, A. Polkovnikov, M. Babadi, T. Oka, and E. Demler, Dynamical Stability of a Many-Body Kapitza Pendulum, Ann. Phys. (Amsterdam) 360, 694 (2015).

[76] M. Bukov and M. Heyl, Parametric Instability in Periodically Driven Luttinger Liquids, Phys. Rev. B 86, 054304 (2012).

[77] J. Berges, K. Boguslavski, S. Schlichting, and R. Venugopalan, Basin of Attraction for Turbulent Thermalization and the Range of Validity of Classical-Statistical Simulations, J. High Energy Phys. 05 (2014) 054.

[78] J. Berges, K. Boguslavski, S. Schlichting, and R. Venugopalan, Universality Far from Equilibrium: From Superfluid Bose Gases to Heavy-Ion Collisions, Phys. Rev. Lett. 114, 061601 (2015).

[79] A. Pineiro Orioli, K. Boguslavski, and J. Berges, Universal Self-Similar Dynamics of Relativistic and Nonrelativistic Field Theories Near Nonthermal Fixed Points, Phys. Rev. D 92, 025041 (2015).

[80] N. Gemelke, E. Sarajlic, Y. Bidel, S. Hon, and S. Chu, Parametric Amplification of Matter Waves in Periodically Translated Optical Lattices, Phys. Rev. Lett. 95, 170404 (2005).

[81] G. K. Campbell, J. Mun, M. Boyd, E. W. Streed, W. Ketterle, and D. E. Pritchard, Parametric Amplification of Atoms, Phys. Rev. Lett. 96, 020406 (2006).

[82] B. M. Anderson, L. W. Clark, J. Crawford, A. Glatz, I. S. Aronson, P. Scherpelz, L. Feng, C. Chin, and K. Levin, Floquet-Band Engineering of Shaken Bosonic Condensates, arXiv:1612.06908 [Phys. Rev. Lett. (to be published)].

[83] S. I. Mistakidis, T. Wulf, A. Negretti, and P. Schmelcher, Resonant Quantum Dynamics of Few Ultracold Bosons in Periodically Driven Finite Lattices, J. Phys. B 48, 244004 (2015).

[84] L. D. Landau and E. M. Lifshitz, Mechanics (Elsevier, Amsterdam, 2008).

[85] I. Carusotto and C. Ciuti, Quantum Fluids of Light, Rev. Mod. Phys. 85, 299 (2013).

[86] This is generically done by applying the kick operator [11] at time $t=0$. However, for this model, the action of the kick operator $K_{\text {kick }}(0)$ reduces to a multiplication by a trivial phase when acting on a Bloch state, so there is, in fact, no need to "rotate."

[87] V. Novicenko, E. Anisimovas, and G. Juzeliunas, Floquet Analysis of a Quantum System with Modulated Periodic Driving, Phys. Rev. A 95, 023615 (2017).

[88] This transformation is formally reminiscent of a standard gauge transformation in electromagnetism, i.e., when going from a gauge where the electric field is written as $\boldsymbol{E}=-\boldsymbol{\nabla} V$ to a gauge where $\boldsymbol{E}=-\mathrm{d} \boldsymbol{A} / \mathrm{d} t$, where $V$ and $\boldsymbol{A}$ denote the scalar and vector potentials.

[89] The quantity $q$ represents the momentum of the corresponding Bogoliubov quasiparticle with respect to the ground state [as becomes clear from the equation 
$\left.a_{n}(t)=a_{n}^{(0)}(t)\left[1+\delta a_{n}(t)\right]\right]$. This will always be tacitly implied in the following, when referring to as mode $q$.

[90] At least for sufficiently high frequency, as considered in the calculations of Ref. [73]; see discussion below concerning the behavior associated with the low-frequency regime.

[91] Slight deviations from this scaling appear only for small values of $K / \omega$.

[92] G. Salerno, T. Ozawa, H. M. Price, and I. Carusotto, Floquet Topological System Based on FrequencyModulated Classical Coupled Harmonic Oscillators, Phys. Rev. B 93, 085105 (2016).

[93] The same analysis holds in the low-frequency regime unless the system is already unstable at low $g$, where a resonance can show up [since $2 E_{\mathrm{av}}(q)=l \omega$ may already have a solution for some $\left.q_{0}, l_{0}\right]$. The instability rate is then no longer governed by the mode $q=\pi$ and the lowest harmonic, but by the mode $q_{0}$ and the harmonic $l_{0}$.

[94] See below for a precise discussion about the validity of this approximation.

[95] We recall that the zeros of $\mathcal{J}_{2}$ are very close to the zeros of $\mathcal{J}_{0}$, but slightly smaller. Moreover, $\mathcal{J}_{0}(x)$ has an additional zero around $x=2.4$, where $\mathcal{J}_{2}(x)$ is not small compared to unity.

[96] Only very small systems with an odd number of sites (thus without featuring a mode $q=\pi$ ) are expected to display differences on the stability boundary.

[97] J. Dalibard, F. Gerbier, G. Juzeliūnas, and P. Öhberg, Colloquium: Artificial Gauge Potentials for Neutral Atoms, Rev. Mod. Phys. 83, 1523 (2011).

[98] N. Goldman, G. Juzeliunas, P. Ohberg, and I. B. Spielman, Light-Induced Gauge Fields for Ultracold Atoms, Rep. Prog. Phys. 77, 12 (2014).

[99] We recall that this singular vanishing of the Bessel function is responsible for a breakdown of the perturbative approach in the $1 \mathrm{D}$ case, which resulted in small disagreement zones (C and D) between the numerical and analytical stability diagrams (Fig. 2).

[100] Equation (40) was derived in the thermodynamic limit. For finite-size systems with open boundary conditions, there are additional corrections that become relevant for $\omega \lesssim 2 J_{\text {eff }}$.

[101] M. Bukov, L. D’Alessio, and A. Polkovnikov, Adv. Phys. 64, 139 (2015).

[102] V. M. Popov, Functional Integrals in Quantum Field Theory and Statistical Physics (Reidel, Dordrecht, 1983).

[103] Y. Castin, Coherent Atomic Matter Waves, edited by R. Kaiser, C. Westbrook, and F. David, Proceedings of the
Les Houches Summer School of Theoretical Physics (EDP Sciences/Springer-Verlag, Berlin, 2001), Vol. LXXII, Chap. "Bose-Einstein condensates in atomic gases: Simple theoretical results."

[104] At least in dimension $d>1$, since the $1 \mathrm{D}$ case is problematic because of the divergence of the quantum depletion in the thermodynamic limit (which is independent of the drive and, hence, exists even at time $t=0$ ).

[105] M. Bukov, M. Heyl, D. A. Huse, and A. Polkovnikov, Heating and Many-Body Resonances in a Periodically Driven Two-Band System, Phys. Rev. B 93, 155132 (2016).

[106] A. Griffin, Conserving and Gapless Approximations for an Inhomogeneous Bose Gas at Finite Temperatures, Phys. Rev. B 53, 9341 (1996).

[107] We implicitly assume here that the system is translational invariant and condensation occurs in the $q=0$ mode. For the general case, please consult the Supplemental Material to Ref. [74].

[108] The Bogoliubov equations [see Eq. (8)] can indeed be exactly rewritten in the form

$$
\begin{aligned}
\partial_{t} F_{11}(t ; q)= & 2 g \operatorname{Im}\left(\left[F_{12}(t ; q)\right]^{*}\right), \\
i \partial_{t} F_{12}(t ; q)= & {\left[\varepsilon_{q}(t)+\varepsilon_{-q}(t)-2 \mu+4 g\right] F_{12}(t ; q) } \\
& +2 g F_{11}(t ; q),
\end{aligned}
$$

where we have defined the correlators in Eq. (46).

[109] The condensate delta peak at $q=0$ is not part of $n_{q}(t)$ since this mode is treated additionally via the field $\phi(t)$.

[110] S. A. Weidinger and M. Knap, Floquet Prethermalization and Regimes of Heating in a Periodically Driven, Interacting Quantum System, arXiv:1609.09089 [Phys. Rev. Lett. (to be published)].

[111] A. Chandran and S. L. Sondhi, Interaction-Stabilized Steady States in the Driven $O(N)$ Model, Phys. Rev. B 93, 174305 (2016).

[112] M. Weinberg, C. Ölschläger, C. Sträter, S. Prelle, A. Eckardt, K. Sengstock, and J. Simonet, Multiphoton Interband Excitations of Quantum Gases in Driven Optical Lattices, Phys. Rev. A 92, 043621 (2015).

[113] There are also other resonances for $\omega=2 \omega_{0} / n$, but they are of higher order, in the sense that both the magnitude of the instability rates and the width of the resonance domain scale as $\alpha^{n}$.

[114] The idea is indeed that the $n$th order correction in $\delta$ will scale as $\alpha^{n}$. 